\title{
Cognition and depression after stroke : course and interaction
}

Citation for published version (APA):

Bour, A. M. J. (2010). Cognition and depression after stroke : course and interaction. [Doctoral Thesis, Maastricht University]. Datawyse. https://doi.org/10.26481/dis.20100916ab

Document status and date:

Published: 01/01/2010

DOI:

10.26481/dis.20100916ab

Document Version:

Publisher's PDF, also known as Version of record

\section{Please check the document version of this publication:}

- A submitted manuscript is the version of the article upon submission and before peer-review. There can be important differences between the submitted version and the official published version of record.

People interested in the research are advised to contact the author for the final version of the publication, or visit the DOI to the publisher's website.

- The final author version and the galley proof are versions of the publication after peer review.

- The final published version features the final layout of the paper including the volume, issue and page numbers.

Link to publication

\footnotetext{
General rights rights.

- You may freely distribute the URL identifying the publication in the public portal. please follow below link for the End User Agreement:

www.umlib.nl/taverne-license

Take down policy

If you believe that this document breaches copyright please contact us at:

repository@maastrichtuniversity.nl

providing details and we will investigate your claim.
}

Copyright and moral rights for the publications made accessible in the public portal are retained by the authors and/or other copyright owners and it is a condition of accessing publications that users recognise and abide by the legal requirements associated with these

- Users may download and print one copy of any publication from the public portal for the purpose of private study or research.

- You may not further distribute the material or use it for any profit-making activity or commercial gain

If the publication is distributed under the terms of Article $25 \mathrm{fa}$ of the Dutch Copyright Act, indicated by the "Taverne" license above, 
Cognition and depression after stroke

Course and interaction 


\title{
"Grow old along with me
}

The best is yet to be

The last of life for which the first was made"

R. Browning

\author{
Cover "Ik herinner me jou..." \\ By A.M.J.J. Bour, in association with I-MediaSolutions \\ Vormgeving F. Peters \\ Production Datawyse, Maastricht \\ ISBN 978-90-75579-46-8
}

(C) Copyright A.M.J.J. Bour, Maastricht juni 2010 


\title{
Cognition and depression after stroke
}

\section{Course and interaction}

\author{
PROEFSCHRIFT
}

ter verkrijging van de graad van doctor aan de Universiteit Maastricht op gezag van de Rector Magnificus, Prof. mr. G.P.M.F. Mols

volgens het besluit van het College van Decanen,

in het openbaar te verdedigen op donderdag 16 september 2010 om 16.00 uur

door

Ariane Marie-José Johannes Bour 


\section{Promotores}

Prof. dr. F.R.J. Verhey

Prof. dr. M. Limburg

\section{Copromotor}

Dr. S.M.C. Rasquin

\section{Beoordelingscommissie}

Prof. dr. R.M.M. Hupperts (voorzitter)

Prof. dr. E.J.L. Griez

Dr. C.M. van Heugten

Dr. R.J. van Oostenbrugge

Prof. dr. B.A. Schmand, AMC, Universiteit van Amsterdam

The research described in this thesis was performed at the Alzheimer Centre Limburg, the School for Mental Health \& Neuroscience (MHeNS), the department of Psychiatry \& Neuropsychology, and the department of Neurology, Maastricht University Medical Centre, Maastricht, the Netherlands.

Financial support by the Netherlands Heart Foundation for the publication of this thesis is gratefully acknowledged.

The publication of this thesis was financially supported by Alzheimer Nederland, Internationale Stichting Alzheimer Onderzoek, Sanofi Aventis, UCB Pharma, Lundbeck, Eli Lilly Nederland BV, Novartis Pharma BV. 


\section{Contents}

$\begin{array}{lll}\text { Chapter } 1 & \text { General introduction } & 7\end{array}$

Chapter 2 Depression after stroke: interaction between neurobiological 19 and psychosocial factors

Chapter 3 The symptomatology of Post-Stroke Depression: comparison 29 of stroke and myocardial infarction patients

Chapter 4 A one-year follow-up study into the course of depression 45 after stroke

Chapter 5 Depressive symptoms and executive functioning in stroke 59 patients. A follow-up study

Chapter 6 How predictive is the MMSE for cognitive performance after 73 stroke?

Chapter 7 The effect of the APOE- $\varepsilon 4$ allele and ACE I/D polymorphism on cognition during a two year follow-up in first-ever stroke patients

Chapter 8 General discussion

Summary 111

Samenvatting

References

Dankwoord

Curriculum Vitae 



\section{General Introduction}




\section{Case history}

Mr. A is a 67 year former grocery shop owner who suffered from a lacunar infarction in the right hemisphere, causing a paresis of the left arm and leg and a slurry speech. He was referred to the emergency department for admittance to the stroke unit. His medical history was unremarkable. He was treated with acetyl salicylic acid therapy and he was prescribed an anti-hypertensive drug. Rehabilitation therapy for his left arm and leg was started and after a few days he was released from hospital and went home with 'good recovery'. Two weeks later his wife complained to the family doctor that her husband was somewhat different. He used to be active before the stroke, but since then, he was tired, apathetic and did not even play with his grand children anymore which formerly gave him great joy. She did not understand why he acted as if he were depressed for he had been so lucky he did not have a severe handicap. When she asked him whether something was wrong or whether he was feeling sad he denied this. The family doctor diagnosed depression and described an anti-depressant.

Some months later a nurse visited the family at home. The wife told her that he had stopped taking the anti-depressive drugs after two months because there was no improvement. The husband still just sat around the house. He denied feeling depressed. His wife told the nurse that he needed help organizing his daily activities and that he had difficulties preparing a cooked meal. She could not send him shopping as he would return with the wrong things.

Several questions arise from this case history. How should the patient's signs and symptoms of apathy be interpreted? What is the course of depression in an (elderly) stroke patient? Can patients be identified who are at a higher risk of cognitive decline after stroke? 


\section{Introduction}

Stroke is the third leading cause of death in The Netherlands and the main cause of permanent disability in the elderly. Each year 41,000 people suffer from a stroke and, to date, approximately 190,000 people are estimated to live with the consequences of stroke [1]. Stroke costs the Dutch society 1.5 billion Euros per year. Stroke incidence which is highly age dependant, will increase as a result of obsolescence. Due to better treatments such as thrombolysis in the acute phase and due to the integrated multidisciplinary care of specialised stroke services life expectancy after stroke has increased substantially over the last twenty years and is still increasing.

Emotional disturbances and cognitive deficits are commonly observed in stroke survivors [2-5]. These have a negative effect on stroke rehabilitation outcome and life expectancy [6-8]. Moreover, they cause a lot of suffering of the patient leading to a reduced quality of life and more health care consumption and costs.

To decrease burden in stroke survivors it is essential that these sequelae are acknowledged and treated in an early phase. Recognition and interpretation of cognitive and psychiatric complaints in stroke patients is difficult. Symptoms often overlap and neuropsychological evaluation in the acute phase after stroke is time consuming and exhausting for the patient. In clinical practice screening measurements that are accurate and easy to administer and interpret are warranted. Furthermore, knowledge of the symptomatology and the course of symptoms may help to identify patients who suffer from these neuropsychiatric disorders or who are at risk for developing them and whether or not they require treatment.

\section{Stroke and neuropsychiatry}

\section{Post-stroke depression}

Depression is the most common psychiatric disorder after stroke. Prevalence varies in the literature substantially, between $10 \%$ and $60 \%$, due to differences between studies of methods, diagnostic procedures and criteria [9]. This immediately raises an important issue. Not only in research but also in daily practice a physician is confronted with a stroke patient who may exhibit complaints that seem not specific for a depression but that are possibly related to the brain damage such as for gastrointestinal complaints or sleeping disorders. 
Cognitive deficits such as psychomotor retardation can also be the direct consequence of the damaging effect of stroke [10-12]. Moreover, stroke patients can exhibit other behavioral disturbances as a direct consequence of the brain lesions caused by stroke such as emotionalism, aprosody, or anosognosy [13-15]. How can post stroke depression (PSD) be diagnosed reliably? What symptoms are discriminative? Diagnosis is especially difficult in patients with disabilities such as aphasia, although nowadays more screening instruments for these patients are available [16].

Is PSD a distinct disease entity characterized by symptoms that all together have a pathophysiology distinct from depression that accompanies other chronic or vascular diseases? PSD was once thought to be the direct consequence of strategic lesions in the brain [13]. Suggested pathogenetic mechanisms are the disruption of frontobasal pathways $[17,18]$ or diffuse vascular brain damage $[19,20]$. This would lead to a 'typical' symptom profile of more cognitive deficits such as psychomotor slowing and executive dysfunction [21, 22]. In order to study the nosological status of PSD, patient samples are often compared to patients suffering from 'functional' depression (i.e., without demonstrable brain pathology). To better understand the role of vascular brain pathology on the appearance of the symptoms seen in PSD the choice of the control group is essential. In particular, the role of non-specific aspects of disease, such as emotional impact, vascular comorbidity, chronicity and level of functioning, that may act as confounder should be controlled when comparing patient groups.

Research into the relationship between depression and vascular disease in the elderly points rather to a bidirectional influence with a large complexity $[23,24]$. Factors other than these biological substrates have pointed out their relevance in the pathogenesis of PSD. Personality traits, such as neuroticism, and environmental factors, such as living alone, have shown to be of relevance and PSD is now considered to be multifactorial in origin $[25,26]$.

The difficulties encountered in explaining the pathophysiology reflect in the treatment strategies of PSD. Little is known about the natural course of depression after stroke [27]. On one hand a substantial number of patients recover within weeks or months whereas on the other hand a very chronic course is reported, especially in patients with a dysthymic condition [28-30]. Are patients who develop symptoms later in stroke recovery similar to patients who develop symptoms directly after the event? Does every patient need a treatment intervention? 
As the origin of PSD is multifactorial there is no 'gold standard' treatment option. In accordance with the biological vascular theories prevention of depression in late life have been reported to be associated with the prevention of vascular disease $[19,20]$. In PSD some beneficial effects of psychotherapy have been reported. There is, however, too little evidence for a beneficial effect and standard utilization of antidepressive agents [31]. This is an issue since PSD can have such a negative outcome and since patients have been reported to benefit from early treatment [32]. In order to make an appropriate treatment decision one should know the benefit and at least should have an idea of the natural disease course what the prospects would be if a patient did not receive this treatment.

Despite the very extensive research into PSD concerning its pathophysiology and epidemiology questions remain to be answered concerning the interpretation of symptoms and the discrimination of patients. Furthermore, more knowledge about the disease course is needed. Investigating one issue also gives answers to other still unanswered questions.

\section{Post-stroke cognitive impairment}

Cognitive deficits are commonly observed after stroke and one in four stroke patients even becomes demented [33,34]. In fact, cerebrovascular disease is the second most common cause of dementia after Alzheimer's Disease (AD). Because of difficulties with the traditional concept of vascular dementia (VaD), which was too narrow to embrace the whole scale of cognitive deficits after stroke, the term vascular cognitive impairment ( $\mathrm{VCl}$ ) was introduced [35]. $\mathrm{VCl}$ describes all levels of cognitive dysfunction due to vascular damage of all etiologies and extents to the brain and thus also encompasses patients who suffer from cognitive decline but do not fulfill the criteria of dementia. As a result of the attribution of a great variability of methods and criteria prevalence rates vary among studies [36, 37]. More than half of stroke patients, however, suffer from $\mathrm{VCl}$ [36, 38-42].

As a result of the location of the stroke the clinical presentation of cognitive deficits is heterogenous [3, 43, 44]. For instance, a stroke located frontotemporally in the cortex of the left hemisphere is likely to cause aphasia whereas a stroke located in the right parietal lobe may cause spatial difficulties. In cognitive decline after stroke and $\mathrm{VCl}$, however, executive functioning, attention, and mental processing speed are typically involved not only in frontal subcortical lesions but also in deficits caused by cortical lesion [45-49]. This heterogeneity in symptomatology hampers the diagnosis in clinical settings. Short bedside instruments for cognitive deficits such as the Mini Mental State Examination 
(MMSE) [50] are generally considered to lack diagnostic accuracy in stroke patients $[51,52]$. However, the MMSE is the most widely used test for cognitive performance in clinical practice and research which has lead to a wide experience and conflicting results as to its utility [53-55]. Although subtle deficits demand extensive neuropsychological examination this often is not achievable in the acute clinical phase. Screening of patients for further detailed neuropsychological testing is preferred since such a test battery is time consuming and demands effort from the often seriously ill patient. Also, in the long term after stroke cognitive deficits can become apparent and should be explored. After the acute hospital setting a trained neuropsychologist often is not available on a routine basis in general care which obliges 'simple' and valid measurement instruments to identify patients in whom detailed testing is necessary.

Traditionally, $A D$ and $\mathrm{VaD}$ have been treated as two distinct disease entities: $A D$ is considered to be caused by the accumulation of neurofibrillary tangles and $\beta$ amyloid plaques in the brain, whereas $\mathrm{VaD}$ is the result of cerebrovascular changes. To date there is growing interest in the interaction between the pathophysiologies of $\mathrm{VaD}$ and $\mathrm{AD}$. This has also warranted a new definition of dementia in relation to vascular pathology. Similarities and overlaps between $\mathrm{VaD}$ and $A D$ are observed in their symptomatology, comorbidity, and pathophysiology. Both $\mathrm{VaD}$ and $\mathrm{AD}$ share traditional vascular risk factors such as hypertension and diabetes mellitus. Moreover, white matter lesions and brain infarctions are independent factors for the clinical presentation of $\operatorname{AD}[38,56,57]$. Patients with $\mathrm{VaD}$ and $\mathrm{AD}$ both show more asymptomatic spontaneous cerebral emboli than controls irrespective of the presence or absence of vascular risk factors or carotid artery obstruction [58]. To make matters more complicated these asymptomatic cerebral emboli are also observed in late-life depression [59].

In the pathogenesis of $A D$ biomarkers such as apolipoprotein E (APOE) play an important role. Some of these biomarkers are reported to be associated with vascular disease and with stroke and $\operatorname{VaD}[56,60-63]$. In patients with this genetic predisposition cognitive deterioration at an advanced age may be caused by an additional development of vascular lesions $[64,65]$. Determining whether or not, and in which way, certain biomarkers have a role in the pathogenesis of cognitive deterioration associated with cerebral vascular disease might help to distinguish patients that are more at risk of (further) cognitive deterioration after a stroke. Furthermore, it perhaps would shed more light on the complex pathophysiologic associations between vascular disease and cognitive decline. More research is needed into this issue. 
Cognitive deficits and depression after stroke are now well established entities. Theories concerning their origin as well as their symptomatology have similarities and overlap. The interpretation of signs and symptoms in daily clinical stroke practice, however, remains difficult. More needs to be learned about the signs and symptoms a patient may show, how they interact and evolve over time, and how they can be measured accurately in daily practice in order to identify patients who suffer from neuropsychiatric sequelae or who are at risk for developing them and to make the best possible treatment decision for the patient.

For this thesis we further researched two cohorts of stroke patients that both have been described and studied earlier $[66,67]$. The main questions and findings are summarized in Table 1.

The cohort we studied in order to examine PSD comprises 190 patients who suffered a first-ever hemispheral infarction [66]. Exclusion criteria were a comorbid intracerebral disease, such as a brain tumor or Parkinson's disease, or psychiatric disorders other than affective disorders, such as schizophrenia or dementia, current depression at the time of stroke, inability to understand the procedures and informed consent due to cognitive dysfunction or aphasia. Patients were followed-up after stroke for 1 year. At one month after the event all patients were administered three depression self-rating scales: the Beck Depression Inventory (BDI) [68], the Hospital Anxiety and Depression Scale (HADS) [69], and the 90-items Symptom Check List (SCL-90) [70]. Then all patients were examined by a psychiatrist using the depression section of the Structured Clinical Interview (SCID-I-R) [71] for DSM-IV and the Hamilton Depression rating scale (HAM-D) [72]. Depression was diagnosed according to the DSM-IV criteria for major and minor depression [73]. Patients were diagnosed as having major depression if they had at least one core symptom (i.e. depressed mood or loss of interest) and at least four other symptoms for at least two weeks. A diagnosis of minor depression was made if a patient suffered from at least one core symptom and at least two but less than four other symptoms. At 3, 6, 9, and 12 months after stroke patients were screened using the three previously mentioned self-rating scales. If a patient scored above the cut-off point on one of these scales he or she was thoroughly examined by a psychiatrist.

The cohort we studied for cognitive deficits after stroke has previously been described as the CODAS study (Cognitive deficits after stroke) [67]. The cohort comprises 194 patients with a first-ever hemispheral stroke. Exclusion criteria were age under 40 years, inability to understand the Dutch language, an initial 


\section{Chapter 1}

MMSE score lower than 15, other comorbid neurological disorders such as a brain tumor, and psychiatric disorders.

Table 1. Summary of the main findings of previous research by our group into Post-stroke Depression and Vascular Cognitive Impairment

\begin{tabular}{|c|c|c|}
\hline Study & Research Questions & Findings \\
\hline \multirow{9}{*}{$\begin{array}{l}\text { Post-stroke } \\
\text { Depression }\end{array}$} & Incidence of PSD in & A cumulative 1-year incidence of major and minor \\
\hline & comparison to depression & depression of $23 \%$ and $15 \%$ respectively [5]. \\
\hline & after MI. & $\begin{array}{l}\text { Higher incidence of PSD explained by higher age, female } \\
\text { sex, and more handicap[5]. }\end{array}$ \\
\hline & $\begin{array}{l}\text { Risk factors and } \\
\text { pathophysiology of PSD. }\end{array}$ & $\begin{array}{l}\text { No support for the lesion-location or vascular } \\
\text { depression hypotheses [74]. }\end{array}$ \\
\hline & & $\begin{array}{l}\text { Female sex, prior depression, level of handicap, and } \\
\text { neuroticism increase the risk for PSD }[25,75] .\end{array}$ \\
\hline & & Hypothesis for a biopsychosocial model of PSD [66]. \\
\hline & Recognition and & The most commonly used instruments (SCL-90, BDI, \\
\hline & treatment of PSD. & HADS, HAM-D) for measuring mood are valid $[9,76]$. \\
\hline & & $\begin{array}{l}\text { PSD is often not recognized and thereby not treated } \\
\text { general practice [77]. }\end{array}$ \\
\hline
\end{tabular}

\begin{tabular}{ll}
\hline Vascular & The phenomenology of \\
Cognitive & VCl. \\
Impairment &
\end{tabular}

The incidence of $\mathrm{VCl}$ was $80 \%[37,42]$.

$\mathrm{VCl}$ is characterized by slowness. Memory is not so often affected in stroke [4] .

Psychiatric disorders co-occur in 50\% [78, 79].

The course of $\mathrm{VCl}$.

Twenty percent of $\mathrm{VCl}$ patients showed a complete remission in cognitive function within two years [80]. Recovery was associated with female sex and higher education [80] .

A diagnosis of mild cognitive impairment ( $\mathrm{MCl}$ ) with impairments in multiple domains had a high sensitivity but low predictive value for dementia after two years [81].

Risk factors of $\mathrm{VCl}$ Territorial infarction, older age, and low educational level increase the risk for $\mathrm{VCl}$ [82].

Patients with progressive brain damage on CT scans perform worse in cognitive functions than patients without progressive damage but in two years cognition stabilized or improved [78].

All patients were followed-up for two years and administered a complete neuropsychological test battery at 1, 6, 12, and 24 months after the event. This battery assessed 10 domains: memory, mental speed, executive functioning, orientation, attention, language, praxis, visuospatial calculation, and reasoning. 
Furthermore, depressive symptoms were measured using the SCL-90 and daily functioning was assessed using the Interview for Deterioration in Daily life in Dementia (IDDD) [83]. Dementia was diagnosed independently by a neuropsychiatrist and a neuropsychologist according to the DSM-IV criteria for dementia [73]. The diagnosis of VaD was based on the NINDS-AIREN criteria [84].

\section{Aims and outline of this thesis}

The aim of this thesis was to study how to identify patients with depression or cognitive deficits in clinical practice. Therefore, the symptomatology and time course of depression and cognitive deficits after stroke were studied. Moreover, the application of routine screening measurements and genetic risk factor identification to facilitate screening patients at risk were assessed.

\section{What factors are of importance in the pathogenesis of depression after stroke?}

Depression is a well known complication of stroke. Initially, it was thought to be a result of focal damage to the frontal regions of the brain caused by the stroke. However, in recent years the focus on the pathogenesis of depression after stroke has shifted towards a multifactorial cause.

In a review article concerning previous investigations by our research group we discuss the influence and interactions of biological, psychological, and social factors on the occurrence of post-stroke depression (Chapter 2). The effect of vascular damage and the localization of a brain infarct on the evolution of depressive symptoms is discussed. Furthermore, a patient's personality traits and social environment are brought into perspective in distinguishing patients who may be at risk for developing a depression after stroke.

Does depression after stroke have a specific symptom profile?

Depression after stroke often remains unrecognized and untreated. The interpretation of emotional and physical signs and symptoms in stroke patients is difficult since many of these symptoms are generally observed in stroke patients. Literature reports are inconclusive. Signs and symptoms of depression in our cohort of stroke patients were compared to patients who suffered a myocardial infarction (MI) who were studied by our research group previously using the same study design (Chapter 3). Stroke and MI share a vascular origin and a sudden, disabling and life-threatening impact on a patient's life. Therefore, the MI cohort was considered appropriate as a control group. 
We hypothesized that if PSD is a disease entity on its own, featured by symptoms provoked by brain damage, then signs and symptoms of depression would be specific and differ from depressive symptoms in MI patients. Signs and symptoms of PSD and post-MI depression were compared and the interaction between depression and stroke on depressive symptoms as measured by the Hamilton Depression Rating Scale was studied.

\section{How does depression after stroke evolve over time?}

Depression after stroke is not a static entity. Not only do depressive symptoms occur in the acute phase after stroke but also after months patients can develop depressive symptoms. Moreover, a certain number of patients will recover. We were curious about the spontaneous course of depression during the first year after stroke and describe its incidence (Chapter 4). We hypothesized that insight into the spontaneous course and prognosis supports, for instance, treatment options. Moreover, we examined whether patients who developed a depression directly after stroke had other characteristics than patients who developed symptoms later hypothesizing that early depressive symptoms have an other causality than late symptoms.

Is the course of depression after stroke influenced by executive dysfunctions and vice versa?

Symptoms of depression and cognitive impairment overlap and interact. Executive dysfunctions are described as a key phenomenon of vascular cognitive impairment and of post-stroke depression. In stroke patients depression and executive dysfunctioning often co-occur. For a patient's treatment and prognosis distinction between these entities is of importance. In this study (Chapter 5) we examined how depressive symptoms measured by the SCL-90 depression subscale and executive dysfunctions as measured by the Stroop and CST relate to each other in stroke and how they evolve with time during the first two years after the event. Moreover, we examined whether patients suffering from depression, executive dysfunctions, or both differed in patient characteristics.

Can the course of cognitive impairment after stroke be predicted and screened for in daily practice by the routinely used Mini Mental State Examination?

Although cognitive deficits are commonly observed in stroke patients it is not feasible or desirable to subject stroke patients to a neuropsychological test battery as a routine for these batteries is time and energy consuming. Daily clinical practice has a need for uniform screening tools. The MMSE is the most widely 
administered test to evaluate cognitive functioning in clinical and research settings. Its validity especially in stroke patients, however, is debated. In this study (Chapter 6) the MMSE was tested using a full neuropsychological test battery in first-ever stroke patients. We examined the ability of the MMSE to screen for cognitive deficits directly after stroke and in the long term. Moreover, we examined whether the MMSE could predict (further) cognitive deterioration with time.

Is it possible to predict course of cognitive deterioration after stroke from a genetic susceptibility?

As in Alzheimers' Disease (AD), which is the most common cause of dementia, research nowadays also reports a genetic predisposition to cognitive decline due to vascular damage. This further supports a concept of a common causality of $A D$ and vascular dementia ( $\mathrm{VaD}$ ) being endpoints of the same disease entity.

We examined whether the Apolipoprotein E epsilon 4 allele (APOE\&4), which is related to $A D$, and the Angiotensin Converting Enzyme DD genotype (ACE- DD), which is reported in $\mathrm{VaD}$, predispose to further general cognitive decline with time after stroke or impairment in the cognitive domains mental speed, executive functions, and memory which are the most commonly affected domains after stroke (Chapter 7). Furthermore, we examined whether there was an interaction between the APOE\&4 allele and the ACE-DD genotype in cognitive functions.

Chapter 8 discusses the findings as presented in this thesis. The strengths and weaknesses of our studies are discussed. Moreover, conclusions that can be drawn from our results are considered and we attempt to provide implications for daily practice and suggest further research concerning neuropsychiatric sequelae after stroke. 



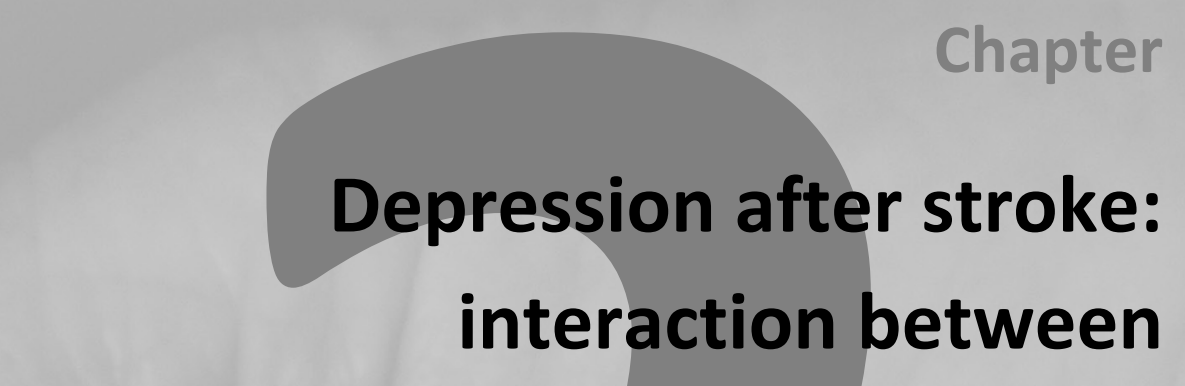

\section{neurobiological and psychosocial}

factors

Adapted from:

I. Aben, A.M.J.J. Bour, F.R.J. Verhey. Depressie na een beroerte: samenspel tussen neurobiologische en psychosociale factoren. Tijdschrift voor Neurologie \& Neurochirurgie. 2007;108(1);3-8 


\section{Chapter 2}

\section{Abstract}

Depression is commonly observed after stroke. Research into post-stroke depression mainly focuses on incidence, phenomenology, course, and risk factors. Particular attention has been paid to biological explanatory models such as the lesion location hypothesis and the vascular depression hypothesis. The role of psychosocial factors in depression however has not had much attention. This review article describes where we are in post-stroke depression research, focusing on the importance of a bio-psychosocial approach. 


\section{Introduction}

Approximately $70 \%$ of stroke survivors have cognitive, emotional, and/or behavioural problems. In about $40 \%$ these problems result in restrictions in daily living [85]. Depression is the most common psychiatric disorder after stroke [15]. Not only does it cause personal suffering, it also negatively influences the revalidation process and it shortens life expectancy $[2,86]$. Despite its clinical importance post-stroke depression often remains unnoticed and untreated [77]. Research into the pathogenesis of post-stroke depression has strongly focused on biological explanations. In this article an overview of recent research into the epidemiology and pathophysiology of post-stroke depression is provided, focusing on the importance of a bio-psychosocial approach.

\section{Epidemiology of post-stroke depression}

Estimates of the occurrence of depression after stroke in the literature vary greatly from $10-64 \%$. This is due to the use of different measurement scales and diagnostic criteria [9]. The utilization of different depression scales and the applicability of the standard diagnostic criteria in this specific group is currently under discussion.

This review compares the results of some investigations, taking 3 potential sources of bias into account. These are the research setting (or the patient population), the diagnostic depression instrument, and the time after stroke the patient was investigated [9]. In community investigations that used standardized diagnostic scales lower prevalence rates were found compared to studies that were performed in patients in hospital or in revalidation settings. Studies which based the diagnosis of depression on self-rating scales often resulted in higher prevalence rates than when a diagnosis was made by a clinician, suggesting that these instruments are of less diagnostic accuracy.

In hospitalized patients the prevalence of 'major depression' (based on a structured interview according to the DSM-III criteria) varied from $19-27 \%$ in the first month after stroke while the prevalence of 'major' and 'minor depression' together varied from 30-47\% [9]. In our cohort study in 190 stroke patients a cumulative 1-year incidence of 38\% ('major depression' $23 \%$ and 'minor depression' 15\%) was found [5]. Most cases of depression occurred within 1 
month after stroke which is in accordance with other prospective studies [29, 30, 87].

\section{Phenomenology}

Post-stroke depression versus 'regular' depression

For the diagnosis of depression after stroke in the literature the standard symptom criteria of a depressive episode according to the DSM are maintained. This given that depression after stroke differs from 'regular' depression in several ways. Lipsey et al. found that psychomotor retardation more often occurred in poststroke depression than in 'functional' depression (69 vs. 30\%), while loss of interest and concentration were slightly less prevalent in the stroke group (70\% vs. 98\%) [88]. Fedoroff et al. compared post-stroke depression to depression in patients who suffered a myocardial infarction and in patients with a spinal cord lesion and reported that anxiety and ideas of reference more often occurred in post-stroke depression [89].

\section{Specificity of somatic symptoms}

Vegetative symptoms such as sleep disturbances, loss of energy or appetite, can occur in both stroke and depression. This also applies to cognitive complaints such as forgetfulness and lack of concentration. How such phenomena should be interpreted diagnostically is under discussion.

Fedoroff et al. found that both psychological and somatic symptom clusters scored significantly higher in depressed $(n=85)$ than in non-depressed $(n=120)$ stroke patients [12]. In a follow-up study these results were consistent over a period of 2 years [11]. Recently, Spalletta et al. reported comparable results [10].

\section{Sensitivity of somatic symptoms}

Of more relevance to a clinician, however, is the question in what way certain symptoms in stroke patients can point to a possible depressive disorder. This concerns the sensitivity. We studied the diagnostic value of the separate items of the Hamilton Depression Rating scale (HAM-D) [90].

Except for suicidal thoughts most psychological symptoms such as hypochondria, lack of disease insight, and feelings of guilt were not very discriminative. Some somatic symptoms such as loss of appetite, psychomotor retardation, and tiredness did have diagnostic value. In practice this means that these phenomena 
should lead to a more extensive psychiatric evaluation for depression and should not be 'explained' directly as a result of the stroke.

\section{Practical guidance}

The results of a stroke can interfere with the signs and symptoms of depression but can also mask depression. Severe aphasia and other cognitive deficits make psychiatric counselling, especially for mood disorders, difficult. An indication for depression in such cases should be obtained by behavioural observations, for instance irritability or a rejecting, withdrawing appearance. Depression can also be falsely suggested by emotional instability (a patient easily cries but does not feel down and maintains his interests) or aprosody (a monotonous speech intonation as a result of a lesion in the left hemisphere).

The differentiation from apathy can also bring difficulties. Apathy can be a part of depression. A negative mood in such a case can lead to apathy (a patient does not feel invited to participate in otherwise pleasant activities and hardly responds to them). However, apathy can also occur without depression. This is frequently observed in combination with cognitive deterioration, especially with problems in executive functions. Often a patient is still able to give a positive response when executive functions are organized for him, therefore an activating approach (day therapy) can be useful. More extensive psychiatric evaluation including an interview with care givers and relatives can make this clear most of the time.

To date there are no well standardized investigational instruments which help to distinguish these behavioural problems from each other in a valid and reliable way. More recently, however, a 'problems checklist' has become available, which can be attributed routinely to all patients several months after a stroke in order to track emotional and cognitive consequences of stroke (CLCE-24) [79, 91]. For a clinician the most important diagnostic tool remains the interview with the patient, thereby thoroughly exploring an almost permanent presence of depressed mood and a generalized loss of interest in particular. In case of doubt or a suspicion of (co morbid) neuropsychiatric disorders a geriatric psychiatrist should be consulted.

\section{Course}

Depression occurring in the first months after stroke often has a favourable course spontaneously: within 1-3 months half the patients improve without any specific 
treatment. If this is not the case depression often takes on a chronic course [28]. In a study performed by Berg and colleagues $46 \%$ of stroke patients who were depressed during the first two months after stroke also appeared to be depressed after 12 and/or 18 months [30]. In our 1-year follow-up study we found comparable results. In the more chronic course prevalence initially tends to increase, in comparison to the community based studies, but tends to increase again after 2 to 3 years [9].

\section{Theories concerning the pathophysiology of post-stroke depression}

\section{Biological models of post-stroke depression}

In the 1980s and 90s there was a lot of support for the hypothesis that depression after stroke depends on left sided and frontally located lesions [13, 18]. A metaanalysis from the literature showed that there appeared to be too little evidence to validate the assumption that left sided strokes more often result in depression that right sided ones [92]. The lesion location theory was then adjusted: the location of the lesion would especially play a role in depression shortly after stroke ('early-onset'). In 'late-onset' depression psychological adaptation difficulties would be of more importance [2]. In spite of extensive scientific research there is only very limited support for the lesion location hypothesis.

In the last few years depression in the elderly has been connected to (generalized) vascular brain damage; the vascular depression. Krishnan and colleagues showed that depression in combination with the presence of white matter damage on MRI is related to higher age, later depression onset, and the absence of psychotic characteristics of depression [20]. A relationship between vascular damage (on $\mathrm{MRI}$ ) and depressive symptoms was also demonstrated in a Dutch community research [93]. Depression can result in a higher risk for cardiovascular disease, but the reverse also applies, by which inflammatory processes could play a role [24]. From our own study comparing 190 stroke patients with 200 myocardial infarction patients the higher incidence of depression appeared rather to be explained by differences between the two groups (age, sex, and level of handicap) than by factors that are specifically stroke related [5]. Hereby it must be pointed out that patients with severe cognitive handicaps such as aphasia and dementia were excluded (for unreliable depression diagnostics). 
Bio-psychosocial model of post-stroke depression

Apart from neurobiological risk factors both the psychological and social consequences of a stroke play a role as pre morbid vulnerability factors in the development of depression, for instance living alone and having a small social circle $[29,94]$. Cognitive deterioration also increases the chance of depression $[133,147]$. In our research pre morbid neuroticism and level of handicap appeared to be the most important independent risk factors for post-stroke depression [75]. General risk factors for depression such as feminine sex, a history of depression, and a positive family history for depression also appeared to be significant predictors, however this mostly concerned analyses in which there were no adjustments for other factors $[95,96]$. This also applies for the effect of 'major lifeevents' [97].The importance of an integrated bio-psychosocial approach concerning the pathophysiology of post-stroke depression also resulted from research from our own country [98]. In Figure 1 we have attempted to illustrate such a multifactorial model.

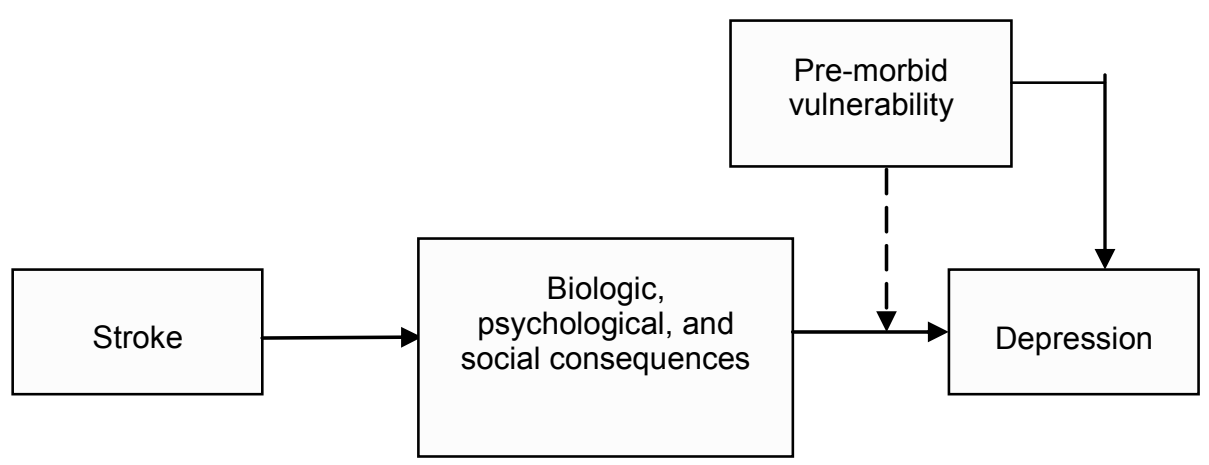

Figure 1. Bio-psychosocial model for post-stroke depression. The cumulative and interactive effects of the biologic, psychological, and social consequences (post-stroke stressors) and pre-morbid vulnerability factors are considered to cause a post-stroke depression.

\section{Treatment}

Given the multifactorial cause of depression after stroke the treatment thereof should also be based on several factors [99]. Treatment which incorporates the patient's ability to copewith the consequences, provides adequate information and (the organization of) real help, thereby taking the patients' personality and coping skills into account, will mostly suffice during the first months, when 
symptoms are not severe. Anti-depressive drugs can lower depressive complaints but a complete improvement often is not achieved by medication alone [100]. Anti-depressive drugs can have specific risks in stroke patients such as epileptic seizures, delirium, and an increased risk of falls. Taking also into account the finding that depression improves spontaneously within 1-3 months after stroke, it is reasonable to assume an attitude of expectation concerning drug therapy.

If drug therapy is chosen then the 'selective serotonin reuptake inhibitors' (SSRIs) citalopram and sertraline are the drugs of first choice because of a more favourable profile of side effects. If these are not sufficiently active then the tricyclic nortriptyline can be chosen because of limited anti-cholinergic effects. Contra-indications should be balanced with care. With citalopram side effects include disturbance of coagulation parameters, gastro-intestinal complaints, or allergy. With nortriptyline heart rhythm disturbances, glaucoma, sedation, or orthostatic hypotension can occur. Furthermore, there is some limited evidence for favourable effects of methylphenidate, especially when apathy is present, and there are some favourable effects described for electro-convulsive therapy.

The treatment of depression after stroke initially can take place in an integrated manner led by a neurologist, nursery home doctor, or own general practitioner, if treatment is not restricted to drug prescription only. Reference to a (geriatric) psychiatrist is required in case of severe depression and/or depression that does not respond sufficiently to the proposed standard treatment.

\section{Conclusion}

Depression after stroke has a cumulative 1-year incidence of 40-50\%. Mostly it occurs in the first few weeks after stroke and tends to have a chronic course in approximately half of cases. Recognition and treatment of post-stroke depression are important because it has a negative interference with rehabilitation outcome measurements, such as daily life functioning and mortality. There is no circumstantial evidence that post-stroke depression has a different symptom profile from other depressive disorders. The differentiation from other complaints and behavioural disorders after stroke can nevertheless be difficult and require an evaluation by a geriatric psychiatrist. Post-stroke depression should not be considered as a purely organic mood disorder but as a result of a complex interaction process of biologic, psychological, and social factors. In the treatment it 
is necessary to strive for influence on these factors. Pharmacological treatment should be initiated in the second instance.

Table 1. Practical recommendations

Every stroke patients should be evaluated after 1-3 months for depressive (and other psychiatric) symptoms. The checklist for the exploration for cognitive and emotional consequences after stroke (CLCE-24) can be of diagnostic utility.

The treatment of depression after stroke is determined individually and should initially consist of information, help in coping and acceptance, and organizing actual help with coping with the handicap.

It is recommended to restrict the prescription of anti-depressive drugs to patients with long lasting or more severe depressive complaints. Certainly in case of milder symptoms a more expecting attitude is entitled.

If an anti-depressive drug treatment is chosen, the SSRIs citalopram and sertraline are of first choice. In combination with anti-coagulants (coumarins) coagulation should be monitored extra. The tricyclic antidepressant nortriptyline is of second choice.

Consulting a geriatric psychiatrist is indicated when symptoms are severe or when standard treatment does not bring sufficient improvement. 



\section{Chapter}

\section{The symptomatology of Post-}

Stroke Depression: comparison of stroke and myocardial infarction

\section{patients.}

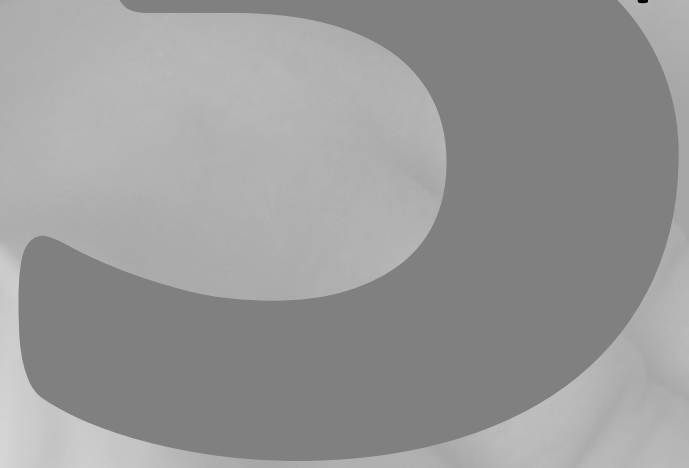

Adapted from:

A. Bour, S. Rasquin, I. Aben, J. Strik, A. Boreas, H. Crijns, M. Limburg, F. Verhey. The symptomatology of Post-Stroke Depression: comparison of stroke and myocardial infarction patients. International Journal of Geriatric Psychiatry. 2009 Oct; 24(10):1134-42 


\section{Abstract}

Background: Depression is a frequent problem in stroke patients but, all too often, the problem goes unrecognized. How depression-like symptoms in post-stroke depression (PSD) should be interpreted is still subject to debate. If PSD has a distinct symptom profile of depression accompanying other chronic vascular somatic conditions then this could imply that PSD is a specific disease entity.

Objective: To study whether depressed stroke patients exhibit other signs and symptoms than patients suffering from depression after myocardial infarction (MI).

Methods: Depressive signs and symptoms were measured using the Hospital Anxiety and Depression Scale and the 17-item Hamilton Depression Rating Scale. The results of 190 stroke patients were compared with the results of $198 \mathrm{MI}$ patients every three months during the first year after the event.

Results: Depressed stroke patients exhibited more loss of interest, psychomotor retardation, and gastro-intestinal complaints as compared to depressed $\mathrm{MI}$ patients. However, in multivariate analyses including both depressed and nondepressed stroke and $\mathrm{MI}$ patients, no specific symptom profile was found to differentiate between the two depressive syndromes by looking at the modifying effect of stroke vs. MI on the occurrence of specific symptoms in depression.

Conclusion: Although in their clinical presentation, depressed stroke patients exhibit a symptom profile different from depressed MI patients, this is not due to differences in the depressive syndrome in these two patient groups but it reflects differences between stroke and MI patients in general. 


\section{Introduction}

Emotional disturbances are commonly observed after stroke $[5,87]$. It is important to recognize post-stroke depression (PSD) as it can have a negative impact on survival, cognitive, and functional outcome [86, 101-103]. Frequency rates described in the literature vary extensively, mostly because of methodological or population differences between studies and because studies use different diagnostic criteria. These differences make comparison difficult. This impedes a better understanding of the pathophysiology and characteristics of PSD.

In particular, it remains unclear whether PSD should be seen as a 'general' depression or as a condition characterized by a specific pathophysiology, symptomatology and course, and whether specific symptoms (for instance tiredness) are related either to depression or to co-morbidity.

In this study, the symptomatology of PSD was of interest. Several studies have been performed to investigate the phenomenology of PSD.

Mostly, symptoms of depressed and non-depressed stroke patients have been compared. Alternatively, comparisons have been made between PSD and primary depression. In order to study symptoms of PSD, the choice of the comparison group is essential. PSD comprises patients who were not depressed before an acute and disabling event, in contrast to patients suffering from primary depression, which can be regarded as a chronic disease caused by various factors.

Depression is also commonly observed after myocardial infarction (MI), which is an acute and life-threatening event, tends to become chronic and can have a great impact on quality of life [104]. Moreover, stroke and MI are similar regarding their pathogenesis since they share a vascular aetiology and risk factors [105].

In this study, the symptom profile for depression observed after stroke was compared to that of depression observed after MI. It was hypothesized that if there is a difference in the symptom profile between the two, this could imply that perhaps PSD is a different disease entity with a specific pathogenesis.

\section{Patients}

In this study, 190 subsequently referred patients with a first-ever hemispheric infarction and 198 patients with first-ever myocardial infarction were included. Population characteristics have been described in detail elsewhere [5]. The 
patients were recruited from the Emergency Department at Maastricht University Hospital in the Netherlands.

Stroke was diagnosed by a neurologist according to the WHO criteria [106]. Computed tomography (CT) differentiated between hemispheric infarction and other causes and types of stroke. Patients with other types of stroke (e.g. recurrent stroke, brainstem stroke or hemorrhage) were excluded from the study in order to increase the homogeneity within each of the two cohorts. Patient data were entered into a prospective stroke registry, which has been described elsewhere [107]. MI was diagnosed by a cardiologist on the basis of evaluation, electrocardiography, and the presence of a maximum value of at least $80 \mathrm{U} / \mathrm{L}$ of the enzyme aspartate aminotransferase (ASAT) [5].

Exclusion criteria were: a) the presence of a major psychiatric disorder other than affective disorders (e.g. dementia, schizophrenia, or a current psychotic episode); b) co-morbid intra-cerebral disease (e.g. a brain tumor or Parkinson's disease); and c) the inability to understand the informed consent procedure (e.g. because of severe aphasia or cognitive dysfunction). All participants provided written informed consent and the study was approved by the local Medical Ethics Committee.

\section{Methods}

Both patient groups were studied using a similar study design and psychiatric assessment. All patients were screened one month after stroke or $\mathrm{MI}$ and were followed-up for the first year following the event. At one month after the stroke or $\mathrm{MI}$, all patients were subjected to a psychiatric assessment conducted by two welltrained research physicians (I.A. and J.S.) using the Structured Clinical Interview of DSM-IV (SCID-I-R) [71]. On the basis of the assessment, the research physicians determined, for each participant, whether the patient suffered no depression, minor depression or major depression. Patients were diagnosed as having major depression if they had one core symptom (i.e. depressed mood or loss of interest) and at least four other symptoms of depression that had lasted for at least 2 weeks. Patients were diagnosed as having minor depression if they had at least one core symptom and two or more but less than five symptoms in total of depression [73]. To determine the severity of the depressive symptoms, the 17item Hamilton Depression Rating Scale (Ham-D) was administered [72]. Furthermore, patients were asked to complete three depression self-rating scales; 
the Beck Depression Inventory (BDI), the 90-item Symptom Checklist (SCL-90), and the Hospital Anxiety and Depression Scale (HADS-A and HADS-D) [68, 69, 108-110]. At the 3, 6, 9, and 12 months' follow-up after stroke or MI, patients were screened for depressive symptoms using the previously mentioned self-rating scales. Patients who exceeded the cut-off values of one of these scales were considered possibly depressed and were re-interviewed by the physician using the SCID-I-R and the Ham-D.

Cut-off values for these rating scales have been defined previously. For the BDI, the cut-off value was $9 / 10$. For the HADS, this was $7 / 8$ for both subscales. With respect to the SCL-90, the cut-off was $22 / 23$ for men and $27 / 28$ for women. If patients scored above the cut-off score on one of these scoring lists, depression was suspected. The sensitivity of this screening procedure has previously been proven to be high. For stroke patients, the sensitivity was $91.2 \%$ and, for $\mathrm{MI}$ patients, it was $85.7 \%[76,111]$.

One month after stroke or MI, disability and handicap were measured using the Barthel Index and Rankin Score.

\section{Statistical analysis}

Cross-sectional prevalence rates for PSD and post-MI depression were analyzed using descriptive statistical methods. Cases with missing data were excluded from the analyses. With respect to the demographic characteristics, comparisons between the two cohorts, Student's t-test was used for continuous variables. For dichotomous variables, the Chi-square test was applied.

In order to test whether stroke patients had a different (overall) score than $\mathrm{MI}$ patients on the Hamilton-D rating scales, mean scores were compared using independent t-tests in depressed and non-depressed subgroups separately. Furthermore, the separate items were dichotomized into 'symptomatology present' or 'symptomatology absent/doubtful'. Items with a scoring range of 0-2 were defined 'symptoms present' if they were scored 1 or 2 . Items with a rating range of 0-4 were defined 'symptoms present' if they were scored 2, 3 or 4 . Then, the presence of signs and symptoms was compared between cohorts in the depressed (e.g. major and minor depressed patients together) stroke and depressed MI patients using Chi-square tests. These analyses were repeated in the non-depressed stroke and MI patients. 
Then, group differences were analyzed by logistic regression analyses. Ham-D items were entered as independent variables and age, gender and Rankin score were entered as dependent variables. To study the influence of stroke or MI on the symptomatology of depression, the interaction between depression diagnosis (depressed /non-depressed) and cohort (stroke or MI) was entered into this model These analyses were only performed for the scorings at one month. No Ham-D scores of the non-depressed stroke and $\mathrm{Ml}$ patients at later follow-ups were available. Then, post hoc analyses of differences in the frequency of the core criteria of depression (depressed mood and anhedonia) based on SCID data were performed using Chi-square tests.

The same analyses were then repeated for the HADS. The HADS subscales for anxiety and depression were studied separately.

For all analyses, statistical significance was defined as $\mathrm{p}<0.05$ (two-tailed). All analyses were calculated using SPSS for Windows version 15.

\section{Results}

From 1997 to 1999, 444 consecutive patients were diagnosed with a first-ever supratentorial cerebral infarction. From this sample, 193 patients were excluded, mainly because of the presence of severe physical or cognitive co-morbidity, death within one month or other reasons such as living too far from Maastricht. Furthermore, 61 eligible patients refused participation. In the end, a total of 190 stroke patients were included in this study.

In the same study period, 415 consecutive patients were diagnosed with a firstever MI. Of these 415 patients, 95 were excluded mainly because of severe comorbidity, death within the first month, or logistic reasons such as not living in Maastricht and the surrounding area or not being traceable. Of the eligible patients, 120 refused participation. Two MI patients suffered a stroke during their hospital admission and were depressed one month after the event. They were also excluded from the analyses. In the end, a total of $198 \mathrm{MI}$ patients were included in this study.

During the follow-up period, more stroke patients than $\mathrm{Ml}$ patients were lost to follow-up. Patients who refused participation were somewhat older in both the stroke and $\mathrm{MI}$ cohort (72.4 vs. $68.8(p=0.01)$ and 65.6 vs. $60.1 \quad(p=<0.0001)$ respectively). Depression scores between non-participants and participants did not 
differ significantly. Detailed information about patient selection and follow-up has been provided in a previous publication [5].

Table 1. Demographic characteristics of stroke and myocardial infarction (MI) patients.

\begin{tabular}{|c|c|c|c|c|}
\hline & & Stroke $(N=190)$ & $\mathrm{MI}(\mathrm{N}=198)$ & $\mathrm{p}$ \\
\hline Sex (female) & & $46.8 \%$ & $23 \%$ & $<0.0001$ \\
\hline Age mean (SD) & & $68.6(11.7)$ & $60.0(10.8)$ & $<0.0001$ \\
\hline \multirow[t]{7}{*}{ Education } & & & & $<0.0001$ \\
\hline & primary school & $42.2 \%$ & $25.5 \%$ & $<0.0001^{*}$ \\
\hline & junior secondary vocational & & & \\
\hline & education & $25.8 \%$ & $19.4 \%$ & \\
\hline & senior secondary vocational & & & \\
\hline & education & $19.0 \%$ & $31.7 \%$ & \\
\hline & (pre-)university education & $13.0 \%$ & $23.4 \%$ & \\
\hline MMSE & & $26(16-30)$ & - & - \\
\hline FAST & & $27(9-30)$ & - & - \\
\hline Barthel mean (SD) & & $17.4(4.7)$ & $20.0(0.2)$ & $<0.0001$ \\
\hline Antidepressants & & $6.2 \%$ & $6.1 \%$ & \\
\hline Rankin mean (SD) & & $2.4(1.2)$ & $1.2(0.7)$ & $<0.0001$ \\
\hline \multicolumn{5}{|l|}{ HADS score (SD) } \\
\hline & Non-depressed§ & $8.1(5.7)$ & $7.0(5.5)$ & 0.10 \\
\hline & Depressed§§ & $16.1(7.5)$ & $15.8(5.7)$ & 0.88 \\
\hline \multicolumn{5}{|c|}{$\begin{array}{l}\text { Hamilton score baseline } \\
\text { mean (SD) }\end{array}$} \\
\hline & Non-depressed** & $7.4(4.8)$ & $7.4(4.3)$ & 0.08 \\
\hline & Depressed*** & $16.3(7.1)$ & $15.5(6.4)$ & 0.61 \\
\hline
\end{tabular}

MMSE: Mini Mental State Examination (minimum score: 0, maximum score: 30);

FAST: Frenchay Aphasia Screening Test (minimum score: 0, maximum score: 30); Barthel Index (completely dependent in Activities of Daily Living: 20, no disabilities: 0); Rankin (no handicaps: 0, bedridden: 5 ).

*Difference between lower (e.g. primary school and junior secondary vocational education) and higher (e.g. senior secondary vocational education) education.

$\S \mathrm{N}=132$ in the stroke cohort and $\mathrm{N}=154$ in the $\mathrm{Ml}$ cohort.

$\S \S \mathrm{N}=31$ in the stroke cohort and $\mathrm{N}=24$ in the $\mathrm{Ml}$ cohort.

** N=142 in the stroke cohort and N=160 in the Ml cohort, respectively, at 1 month after the event.

*** $\mathrm{N}=40$ in the stroke cohort and $\mathrm{N}=26$ in the Ml cohort, respectively, at 1 month after the event.

The characteristics of both cohorts are summarized in Table 1. Stroke patients were older, less educated, and more often female than MI patients. Stroke patients showed more physical handicap and were more severely disabled in their daily activities. Stroke patients were more often in a nursing care facility than MI patients, albeit not significantly. Institutionalized stroke patients were slightly more often depressed than stroke patients living at home, albeit not significantly. 
After one month, 41 (21.6\%) of the stroke patients and $26(13.2 \%)$ of the $\mathrm{MI}$ patients were diagnosed as suffering from major or minor depression $(p=0.05)$. Major depression was more frequently diagnosed in the stroke cohort than in the MI cohort (13.2\% and $7.0 \%$ of the depressed patients, respectively). However, this difference was not significant.

In $38.5 \%$ and $50 \%$ of depressed stroke and $\mathrm{MI}$ patients at 1 month the depression was persistent at three months. Major depression in stroke and myocardial infarction was averagely present at 2.0 and 2.5 consecutive follow-up assessments respectively. Minor depression was averagely present in 1.1 and 1.5 follow-up assessments. These differences in duration were not significant. One month after the event, the mean overall score on the Hamilton Depression Scale was 16.3 (SD 7.1) for depressed stroke patients and 15.8 (SD 6.3) for depressed MI patients. Non-depressed patients showed a mean score of 7.4 in both cohorts (SD 4.8 in the stroke cohort and SD 4.3 in the MI cohort, respectively).

After one month, non-depressed stroke patients showed more feelings of guilt ( $7.4 \%$ and $0.6 \%$ of the non-depressed stroke and $\mathrm{MI}$ patients, respectively; $\mathrm{p}=0.001)$, loss of interest $(57.7 \%$ and $10.7 \%$, respectively; $\mathrm{p}<0.0001)$, and psychomotor retardation ( $14.1 \%$ and $2.9 \%$, respectively; $p<0.0001$ ) on the Ham-D. Non-depressed MI patients exhibited more middle-of-the-night insomnia (26.2\% and $40.7 \%$, respectively; $\mathrm{p}=0.001)$, tiredness $(59.7 \%$ and $82.5 \%$, respectively; $p=<0.0001)$, and sexual complaints ( $10.5 \%$ and $30.2 \%$, respectively; $p<0.0001$ ).

Figure 1 shows the percentage of depressed stroke and MI patients who scored complaints as 'present' on each Hamilton item separately at 1, 6, and 12 months after the event, respectively. After one month, depressed stroke patients experienced more often depressed mood $(82.9 \%$ and $61.5 \%$ of the depressed stroke and $\mathrm{Ml}$ patients, respectively; $\mathrm{p}=0.05)$, suicidal thoughts $(24.4 \%$ and $3.8 \%$, respectively; $p=0.028)$, loss of interest $(87.8 \%$ and $53.8 \%$, respectively; $p=0.002)$, psychomotor retardation $(48.8 \%$ and $11.5 \%$, respectively; $p=0.002)$, and gastrointestinal symptoms $(61.0 \%$ and $30.8 \%$, respectively; $p=0.016)$, whereas depressed $\mathrm{MI}$ patients experienced more sexual disturbances $(20.0 \%$ and $57.7 \%$ respectively; $p=0.002)$, and showed less insight into their disease $(9.8 \%$ and $34.6 \%$, respectively; $p=0.012$ ). 

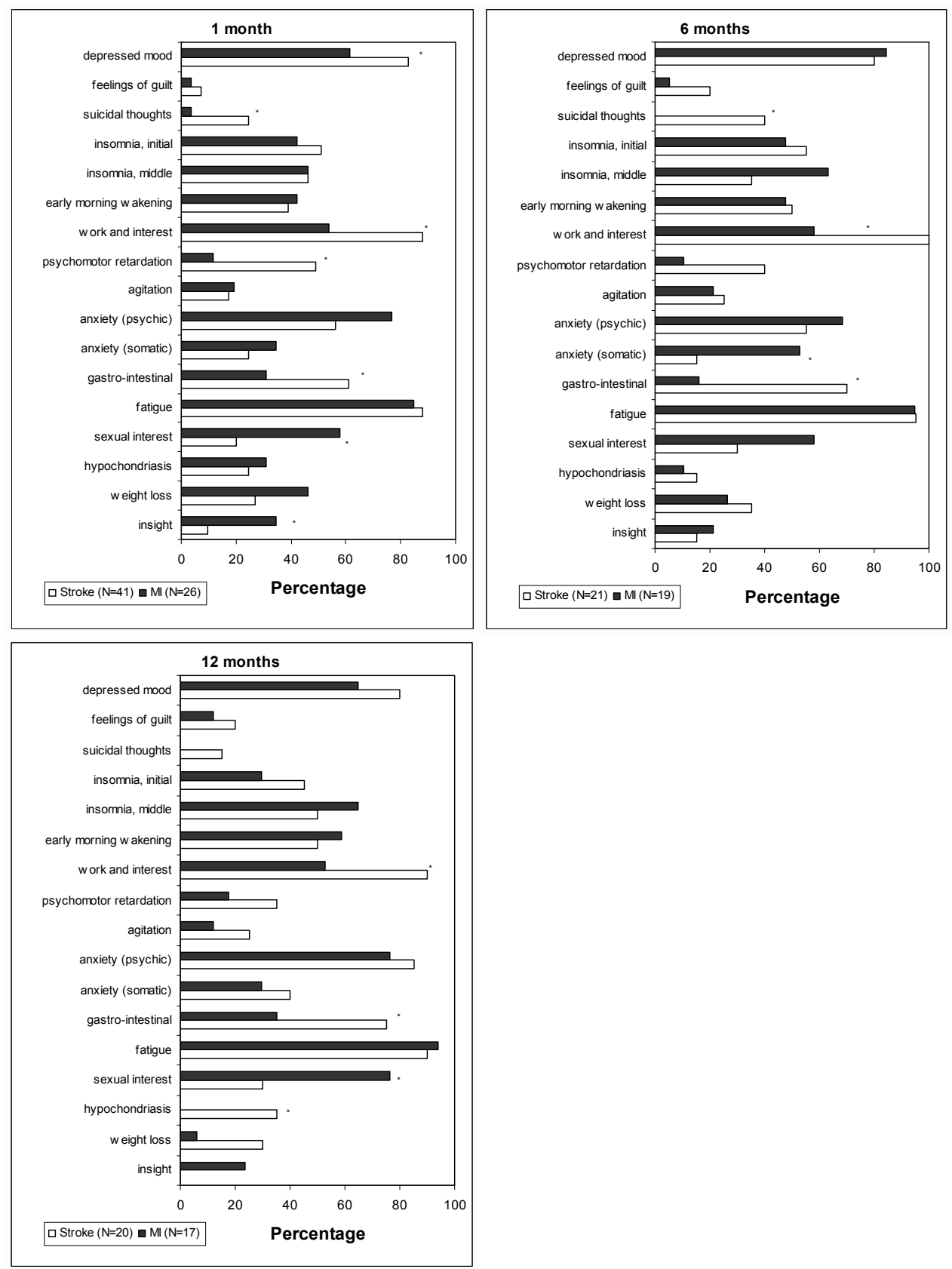

Figure 1. Percentage of depressed stroke and MI patients with complaints on the Hamilton 17 Item Depression Scale. ${ }^{*} p<0.05$ 
Using logistic regression analyses including data from both depressed and nondepressed patients, the group differences (stroke vs. MI) in loss of interest, psychomotor retardation, sexual disturbances, and insight remained significant after adjustment for age, gender, and Rankin score. However, after entering the diagnosis (depressed/non-depressed) and the interaction cohort*diagnosis into the model, none of the above mentioned symptoms showed to be specifically associated with depression in either stroke or MI. Subsequent re-analyses by removing the interaction term from the equation revealed that loss of interest was the only symptom that occurred significantly more frequently in stroke patients (independent from a diagnosis of depression). Furthermore, the diagnosis of depression was based on the symptom criteria for both depressed mood and loss of interest (according to the SCID) in $63.4 \%$ and $30.8 \%$ of depressed stroke and $\mathrm{MI}$ patients, respectively, while in $17.1 \%$ of depressed stroke and $38.5 \%$ of depressed MI patients, it was based on the symptom criterion depressed mood only and in $19.5 \%$ of depressed stroke and $30.8 \%$ of depressed MI patients, it was based on the symptom criterion loss of interest only. $\left(\chi^{2}(d f=2)=7.1, p=0.02\right)$.

Comparisons between depressed stroke and MI patients at 3, 6 and 12 months revealed similar findings to those at 1 month after stroke or MI.

Table 2 shows the mean scores for each cohort separately for the HADS anxiety and depression subscales at each follow-up. The overall HADS scores were, for the depressed stroke patients, slightly higher, albeit this result was not significant (Table 2). Depressed stroke patients attained a higher score on the HADS-D, whereas MI patients attained a higher score on the HADS-A. However, only the difference in the depression subscale after six months was significant (11.0 for the stroke cohort and 8.3 for the Ml cohort; (t-test, $\mathrm{p}=0.009$ ) but this effect disappeared after correction for age, gender, Rankin score, and diagnoses. Moreover, there was no interaction effect (cohort*diagnosis). 
Table 2. Mean scores of Hospital Anxiety and Depression Subscales (HADS) for depressed (D) patients after stroke and myocardial infarction (MI).

A. HADS: Anxiety Subscale.

\begin{tabular}{lllllll}
\hline & Stroke & \multicolumn{5}{c}{ MI } \\
month & D & Major & minor & D & major & minor \\
\hline 1 & 7.24 & 7.72 & 6.45 & 8.23 & 9.73 & 6.70 \\
3 & 9.00 & 11.0 & 7.00 & 10.4 & 10.8 & 9.75 \\
6 & 9.47 & 10.57 & 6.40 & 8.32 & 9.60 & 6.89 \\
9 & 7.38 & 8.76 & 4.17 & 8.14 & 8.85 & 7.23 \\
12 & 7.44 & 8.82 & 5.29 & 8.38 & 9.86 & 7.22 \\
\hline
\end{tabular}

B. HADS: Depression Subscale.

\begin{tabular}{lllllll}
\hline & Stroke & & & MI & \\
month & D & major & minor & D & major & minor \\
\hline 1 & 8.97 & 9.32 & 8.35 & 7.58 & 8.49 & 6.50 \\
3 & 10.37 & 12.22 & 8.52 & 9.40 & 9.25 & 9.63 \\
6 & 11.03 & 11.76 & 9.00 & $8.26^{*}$ & 9.90 & 6.44 \\
9 & 9.65 & 9.93 & 9.00 & 8.52 & 10.28 & 6.27 \\
12 & 9.39 & 9.45 & 9.29 & 9.63 & 10.42 & 9.00 \\
\hline
\end{tabular}

$\mathrm{p}<0.05$

Mean scores for the HADS-A and HADS-D subscales for depressed stroke and MI patients (D; e.g. major and minor depression) and subdivision in major depression and minor depression according to the DSM-IV criteria.

\section{Discussion}

In this study, the clinical symptomatology of depressed stroke patients was compared to that of depressed MI patients every three months after the stroke or MI.

During the follow-up period of 12 months, almost $40 \%$ of stroke patients and almost $30 \%$ of $\mathrm{MI}$ patients were diagnosed at one time as suffering from major or minor depression.

Depressed stroke patients exhibited more loss of interest, suicidal thoughts, psychomotor retardation, and gastro-intestinal complaints. In contrast, $\mathrm{MI}$ patients showed more anxiety, loss of sexual interest, and loss of insight into their illness. Both depressed stroke patients and depressed MI patients frequently suffered from fatigue. This profile was persistent over all follow-up assessments. 
Using a multivariate statistic approach including both depressed and nondepressed patients and adjusting for age, gender and level of handicap, only group differences for loss of interest and psychomotor retardation remained significant. However, we found no modulating effect of stroke or MI on the symptomatology of depression (by studying the interaction between cohort (stroke vs. MI) and diagnosis (depressed vs. non-depressed). This indicates that stroke itself has a more pronounced influence on the pathogenesis of these symptoms in depressed patients than depression. Non-depressed stroke patients showed a similar profile albeit that in non-depressed patients these symptoms were less severe.

Cognitive, psychological and autonomic deficits are frequently observed in poststroke depression and in depression that is not related to stroke. However, these deficits may be due to the direct brain-damaging effect of the stroke itself. For instance, in this study, psychomotor retardation and loss of interest could be interpreted as apathy, which is often found in stroke patients [112-116]. Although depressed stroke patients more frequently suffered from anhedonia than MI patients, most of these patients had a lowered mood in addition to loss of interest based on the SCID-I-R findings. Therefore, it is not probable that we misdiagnosed apathy as a condition that is distinct from depression. It could rather be argued that stroke patients were more severely depressed. However, although more pronounced in major depression, we found the coexistence of mood disturbance and loss of interest also in the minor depressed. Furthermore, severity of depression as measured by the Hamilton and the HADS did not differ between stroke and MI patients. Our findings indicate that when diagnosing post stroke depression it should be considered that stroke patients often suffer from autonomic and cognitive complaints which possibly mimic a depression. As in patients with depression due to other causes, examining mood is of diagnostic importance in stroke patients.

In stroke patients, interpretation of neuropsychological and physical symptoms to differentiate depressed from non-depressed patients remains difficult. Fedoroff et al. [89] compared the symptomatology of 44 depressed stroke patients to 25 and 12 depressed patients with $\mathrm{MI}$ and acute spinal cord injury, respectively. They found significantly more anxiety symptoms in the stroke population. In this study, MI patients more often showed signs of anxiety. After adjusting for confounding factors, this difference was, however, not significant.

Symptoms of depression have been previously compared to symptoms in nondepressed stroke patients or patients with a functional depression. Lipsey et al. [88] and Andersen et al. [87] found that PSD had no specific features when 
compared to functional depression. Spalletta et al. [10] found a higher frequency of depressed mood, lack of interest, fatigue, insomnia, and psychomotor agitation in depressed stroke patients when compared to non-depressed stroke patients. In another study, 124 patients were assessed either 2, 6, or 12 months after stroke and then compared to healthy subjects. They found a higher frequency of depression, irritability, eating disturbances, agitation, apathy, and anxiety in stroke patients. In the post acute phase, patients exhibited essentially more apathy and night-time disturbances [117]. Paradiso et al. [11] found more vegetative and psychological symptoms in patients with post-stroke depression as compared to non-depressed stroke patients throughout a follow-up period of two years. Autonomic anxiety, morning depression, fatigue, and loss of interest were more frequently reported throughout the period of two years, whereas loss of libido, self-depreciation, feelings of guilt, and irritability decreased over time. Tateno et al. [118] found that patients with early-onset post-stroke depression had more vegetative symptoms than patients with late-onset post-stroke depression. Gainotti et al. [119] found no differences between early and late onset depressive symptoms after stroke. Compared to patients suffering from endogenous depression, they found more anxiety, catastrophic reaction, hyper-emotionalism, and reactive diurnal mood variations in post-stroke depression whereas patients with functional depression showed more suicidal thoughts and anhedonia. These studies do not differentiate how symptoms of post stroke depression are related to stroke. Somatic disease often is related to depression in later life and the accompanying symptom profiles differ between disease causes and also from functional depression [120,121]. Therefore, in the comparison of depressive signs and symptoms in post stroke depression the choice of the control group is essential. In this study, the symptomatology of depressed stroke patients was compared to depressed patients suffering from $\mathrm{MI}$, which is also an acute life threatening event. Furthermore, stroke and MI both share vascular risk factors and pathophysiology, which are related to depression [105, 122, 123].

There are some limitations of this study that should be mentioned. First of all, demographic characteristics between the two cohorts differed significantly. Stroke patients were more often female, lower educated, and older. As stated before, the risk of developing a post-stroke depression or depression after $\mathrm{MI}$ is dependent not only on biological factors but also on psychosocial factors $[25,124,125]$. To compensate for this limitation, the analyses were adjusted for these potential confounders. It is conceivable that these characteristics influence symptoms differently between the two patient groups. Matched patient cohorts would be 
preferred to rule out the influence of such confounders. However, this was not possible due to the design of our study which included consecutive first-ever stroke and MI patients who visited the emergency department. By doing so our cohorts do represent daily clinical practice.

Furthermore, we excluded severely aphasic and cognitively impaired patients. So, this study population contained the cognitively better part of stroke patients as this generally applies to studies that require long-term cooperation. Since level of handicap and cognitive dysfunction are predictive of depression, we cannot exclude the possibility that the prevalence of depression among patients is actually higher, especially in the stroke cohort, or that certain symptoms have a higher prevalence. In addition, there were more non-participants in the stroke cohort than in the $\mathrm{Ml}$ cohort. Therefore, the two cohorts may differ with regard to selection bias. Non-participants did not differ in prevalence or severity of depressive symptoms from participants at the time of patient recruitment.

In literature general depression self-rating scales in stroke such as the HADS are debated [126]. In stroke patients administration of stroke specific depression scales is preferable [16]. General depression scales are commonly used in research and sufficient validity in stroke patients is reported $[127,128]$. In our study we used self-rating scales (BDI, SCL-90, and HADS) to screen for signs and symptoms that could be due to a depression. Diagnosis however was based on DSM-IV criteria for depression using the SCID-I-R, which is the gold standard in diagnosing depression. Since depression in stroke patients was compared to depression in patients not suffering from stroke (MI patients) we administered these general scales. By doing so the incidence of depression in stroke patients in our study perhaps is underestimated because of insufficient sensitivity. However, we studied the validity of these three self-rating scales in a previous study and found these scales to be highly accurate in the screening for depression in stroke patients [76]. In this study no distinction was made between patients suffering from major or minor depression since the severity of depressive symptoms is considered to be a continuum rather than two entities.

In this study, depressed stroke patients showed more often loss of interest, psychomotor retardation, and vegetative symptoms when compared to another group of depressed patients with a sudden, life-threatening, vascular disease. Although the pathophysiology of stroke itself is related to the pathogenesis of these symptoms a clinician should consider that they can conceal depression since these symptoms are more severe in depressed than in non-depressed patients. Although the difference between apathy and anhedonia due to depression can be 
difficult, accurate diagnosis is achieved when lowered mood is considered. Because diagnosis and interpretation of possible depressive symptoms in stroke patients is difficult, these patients should be evaluated by well-trained physicians, taking individual patients' predisposing factors such as personality traits and information given by carers or family into account. Furthermore, stroke specific diagnostic measurements for depression after stroke are advisable [129] and future studies should be directed to developing more specific rating scales and diagnostic criteria for depression in stroke patients, including patients suffering from aphasia. In the diagnosis of post stroke depression insight into the specificity and predictive value of symptoms would be interesting. It's important to have a better understanding of the course of depressive symptoms in time to have a better understanding of how long after the event patients should be screened and perhaps treated.

It must be considered that, in stroke patients, vegetative, cognitive, and psychological signs can conceal a depression [10,90]. This should especially be considered during the rehabilitation process since the susceptibility to negative symptoms and lack of initiative may explain why stroke patients have difficulties rehabilitating and why stroke tends to have a high morbidity and mortality rate. 



\section{A one-year follow-up study into the course of depression after stroke}

Adapted from:

A. Bour, S. Rasquin, I. Aben, A. Boreas, M. Limburg, F. Verhey. A one-year follow-up study into the course of depression after stroke. Journal of Nutrition, Health \& Aging. 2010; 14 (6): 488-493 


\section{Abstract}

Background: Stroke patients commonly suffer from neuropsychiatric disorders, such as depression, that negatively influence stroke outcome. Diagnosis, treatment and prevention of post-stroke psychiatric disorders including depression are under debate.

Objective: To study the course of depression after stroke.

Methods: One hundred and ninety first-ever stroke patients were screened for depressive symptoms at $1,3,6,9$, and 12 months after stroke. Diagnosis of depression was made according to the DSM-IV criteria of major and minor depression.

Results: Follow-up was completed in 138 patients. The cumulative incidence of post-stroke depression (PSD) in 1 year was $36.2 \%$. One month after stroke the prevalence of PSD was $18.8 \%$. Thirty percent of patients who were depressed in the first three months did not reach cut-off levels on depression screening instruments at the following assessments. In $44 \%$ of these patients symptoms recurred. Recurrent cases were older than patients with limited disease. In $40 \%$ of PSD patients depression persisted for at least two consecutive following follow-up visits. Persistent cases were more disabled and suffered more often from major depression.

Conclusion: Half of PSD patients become depressed within the first month after stroke. Although most patients recover, a clinician has to be aware that symptoms can recur especially in older patients and that in patients with major depression symptoms may be persistent. In these patients treatment should be considered, whereas in patients with limited disease an observational approach may suffice. 


\section{Introduction}

Not only does stroke cause physical inability but it also has essential emotional and cognitive consequences which can be severely debilitating. Depression is among the most commonly reported neuropsychiatric complications of stroke. In the long term, depressive symptoms are highly prevalent in stroke survivors $[2,117,130$, 131]. Depression has a negative impact on stroke mortality, recovery, and quality of life in stroke survivors [8].

Identification of patients at risk focuses on bio-psychosocial risk factors such as female sex, living alone, older age, level of handicap, and a neurotic personality, but study findings are inconsistent [29, 30, 132-134]. The anatomical influence of lesion size or location as a risk factor is controversial [18, 92]. For the optimal identification and treatment of PSD patients knowledge about the course of depressive symptoms after stroke is of necessity. How PSD evolves over time remains to be explored more extensively. Despite much research into PSD only a few studies focus on the natural course and reported results are inconsistent. Most of these studies comprise long follow-up periods with long intervals between assessments. Some studies state that PSD most frequently develops shortly after stroke but that it also can appear months or years after the event. Patients who develop PSD shortly after the event may differ in characteristics and symptomatology from patients in whom PSD develops later. Tateno et al. state that early symptoms of PSD are associated with the direct consequences of stroke, whereas late symptoms are more related to psycho-social functioning [118]. Some studies demonstrate that most cases of PSD recover spontaneously within months without any treatment whereas other studies report that depression also may take a chronic course [27, 29, 30, 135]. Knowledge of risk factors enables a clinician to identify patients who are at risk of developing a more chronic course of PSD and who would benefit from intervention. However, for efficient management of PSD, knowledge about the natural course of symptoms is warranted, especially since the effects of treatment of PSD with anti-depressive drugs and nonpharmacological interventions are debatable [136, 137]. The aim of this study is to describe the natural course of depression after stroke in first-ever stroke patients. We hypothesized that patients in whom depression takes a chronic course are more severely affected and have more risk factors for developing PSD than patients in whom PSD recovers spontaneously in the short term. Furthermore, we were interested whether patients with early PSD differed from patients who develop PSD later in the course of stroke recovery. 


\section{Patients}

In this study 190 consecutive patients with a first-ever hemispheric cerebral infarction and without depression in the weeks prior to the infarction were included. The patients were recruited from the Emergency Department of the University Hospital of Maastricht, The Netherlands. Patients and study design have been described previously [5]. In short, first-ever ischaemic stroke patients were examined every three months after stroke in a prospective longitudinal study design during the first year after stroke.

Stroke was diagnosed by a neurologist according to the WHO criteria [106]. Computed tomography (CT) differentiated between hemispheric infarction and other causes and types of stroke. If patients had another type of stroke (e.g. recurrent stroke, brainstem stroke or haemorrhage) they were not included in order to increase homogeneity of groups. Patient data were entered into a prospective stroke registry, described elsewhere [107].

Exclusion criteria were any major psychiatric disorder other than a depressive disorder (e.g., schizophrenia or a current psychotic episode), the onset of a current depressive episode before stroke, co-morbid intracerebral disease (e.g., a brain tumour or Parkinson's disease), and inability to understand the informed consent procedure (e.g., because of severe aphasia or cognitive dysfunction). Furthermore, in order to obtain a homogenous population patients with other stroke types such as haemorrhage, recurrent stroke, or brainstem infarction were excluded from the study. All participants gave written informed consent and the study was approved by the local Medical Ethics committee.

\section{Assessments}

One month follow-up after stroke all patients had a psychiatric assessment by a well-trained research physician (IA) using the Structured Clinical Interview of DSMIV (SCID-I-R), to diagnose them as non-depressed, major or minor depressed. To determine severity of depression and symptoms, the 17-item Hamilton Depression Rating Scale (Ham-D) was administered [72]. Furthermore, patients were asked to fill out three depression self-rating scales, i.e. the Beck Depression Inventory (BDI) [68], the 90-item Symptom Checklist (SCL-90) [70], and the Hospital Anxiety and Depression Scale (HADS-A and HADS-D) [69]. Cut-off values for these rating scales and their sensitivities have been defined previously [76]. For the BDI the cut-off 
value was $9 / 10$, for the HADS $7 / 8$ for both subscales, and for the SCL-90 the cut-off was $22 / 23$ for men and 22/28 for women.

At 3, 6, 9, and 12 months after stroke, patients were screened for depressive symptoms using the previously mentioned self-rating scales. Patients who exceeded the cut-off values on one of these scales were re-interviewed by the physician using the SCID-I-R and Ham-D for diagnosis.

Neuroticism was evaluated 1 month after stroke using the Dutch translation of the Neo Five Factor Inventory (NEO-FFI) $[138,139]$. This is a self-report questionnaire consisting of 60 statements comprising the five main domains of personality: neuroticism, extraversion, openness to new experiences, agreeableness, and conscientiousness. Each statement is rated on a 5-point scale ranging from 'strongly disagree' to 'strongly agree', resulting in a total dimension score of 12-60. Neuroticism is defined as the tendency to experience negative emotions and to have poor coping skills and it is highly associated with psychopathology, including depression [140].

Disability and handicap were measured at baseline using the Barthel Index and Rankin Score. Global cognitive functioning and aphasia were determined at baseline by the MMSE and FAST. Patients who were excluded from the study or who refused participation were not assessed.

\section{Statistical analyses}

For demographic variables descriptive analyses were administered. Survival techniques were used to study the cumulative incidence.

Cases with persistent depression were defined as having a diagnosis of depression during at least 2 consecutive follow-up visits and who reported symptoms of depression between these visits. Cases were defined as transient if they were depressed at one follow-up. Recurrent cases were diagnosed with depression during more than one visit, with sub-threshold symptom levels in between.

Early onset depression was defined as a diagnosis of depression at 1 month. Late onset depression was defined as a depression that debuted at subsequent followup visits after $3,6,9$, or 12 months.

To compare descriptive characteristics Student's t tests were applied in the case of continuous variables and Chi-square tests were used in the case of dichotomous variables. For non-parametrically distributed characteristics Mann-Whitney $U$ tests were applied. 
For the comparison of group variances between non-depressed, persistent, transient, and recurrent cases one-way ANOVA was applied with post-hoc Bonferroni and Scheffé correction in the case of significance in between-group variances. In the case of not -normally distributed characteristics, Kruskall-Wallis tests were applied. In the case of dichotomous variables Chi-square tests were applied.

To study the relationships between cognitive and functional deficits and depression, correlation coefficients were estimated for the MMSE, FAST, Barthel Index, and Rankin score.

All statistical analyses were performed using the Statistical Package for Social Sciences version 15 (SPSS15).

\section{Results}

Patients were selected from 444 consecutive patients who visited the emergency department with a first-ever hemispheral infarction. One hundred and ninetythree patients were excluded (37 died within 1 month after stroke, 54 patients had severe aphasia or other cognitive deficits, 38 had severe somatic co-morbidity, 12 had combined somatic and cognitive co-morbidity, 12 patients had a current psychiatric disorder including depression, 40 were excluded because of miscellaneous reasons such as living too far from the hospital, not native Dutch speaking or untraceability), and 61 patients refused participation. Of the 190 patients who entered the study at baseline, 52 were lost to follow-up during the 1 year follow-up period (11 died, 27 refused further participation, 14 were incapable of further participation or untraceable). Table 1 shows the population characteristics. Patients lost to follow-up were older, lower educated, had lower MMSE scores and were more handicapped. There was no difference in prevalence of depression. 
Table 1. Demographic characteristics

\begin{tabular}{llll}
\hline & $\begin{array}{l}\text { Total cohort } \\
(\mathrm{N}=190)\end{array}$ & $\begin{array}{l}\text { Patients with complete } \\
\text { follow-up (N=138) }\end{array}$ & $\begin{array}{l}\text { Drop-outs } \\
(\mathrm{N}=52)\end{array}$ \\
\hline Sex (F) & $46.8 \%$ & $48.1 \%$ & $55.1 \%$ \\
Age mean (SD) & $68.6(11.7)$ & $66.1(11.4)$ & $75.1(9.7)^{*}$ \\
Education (high) & $32.0 \%$ & $37.7 \%$ & $17 \% *$ \\
MMSE median (range) & $26.0(16-30)$ & $27.0(19-30)$ & $25.0(16-30)^{*}$ \\
FAST median (range) & $27.0(9-30)$ & $26.0(4.1)$ & $23.8(4.8)^{*}$ \\
Barthel median (range) & $20.0(0-20)$ & $18.0(0-20)$ & $20.0(1-20)^{*}$ \\
Rankin mean (SD) & $2.4(1.2)$ & 2.2 & $2.9 *$ \\
Hamilton score mean (SD) & $9.5(6.5)$ & $8.7(6.4)$ & $11.5(6.4)$ \\
PSD & $35.8 \%$ & $36.2 \%$ & $36.2 \%$ \\
Neuroticism mean (SD) & $30.1(7.3)$ & $30.0(6.8)$ & $30.4(9.1)$ \\
Cortical infarction & $44.9 \%$ & $44.9 \%$ & $45.1 \%$ \\
Left-sided lesion & $46.8 \%$ & $44.9 \%$ & $51.9 \%$ \\
History of depression & $21.5 \%$ & $21.3 \%$ & $22.0 \%$ \\
Family history of depression & $20.1 \%$ & $19.4 \%$ & $22.2 \%$ \\
\hline
\end{tabular}

MMSE: Mini Mental State Examination (minimum score: 0, maximum score: 30 );

FAST: Frenchay Aphasia Screening Test (minimum score: 0, maximum score: 30); Barthel Index (completely dependent in Activities of Daily Living: 20, no disabilities: 0); Rankin (no handicaps: 0, bedridden: 5$)$. ${ }^{*} p<0.05$ : for comparisons between patients who completed the follow-up and dropouts.

Table 2 shows the cumulative incidence and prevalence rates of PSD at each assessment during the follow-up period of 1 year. In total, 50 patients were diagnosed with depression during the follow-up (1 year cumulative incidence of $36.2 \%)$. Thirty patients were diagnosed suffering from major depression and 20 patients were diagnosed suffering from minor depression. In 52\% of PSD patients depression was already apparent after 1 month. In 14 patients (28\%) depression was recurrent. 

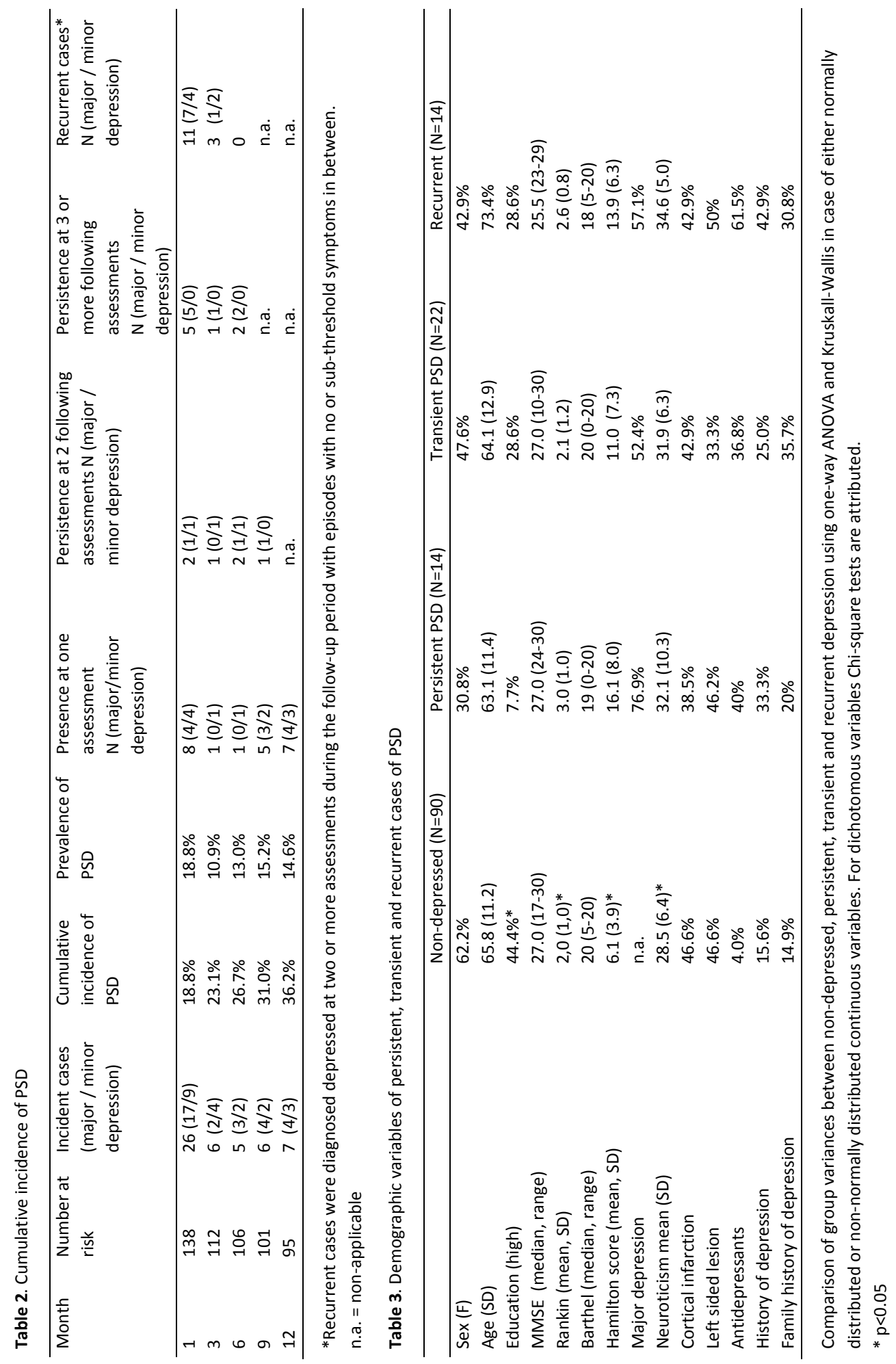
Table 3 shows the characteristics of non-depressed patients and patients with persistent, transient and recurrent depression. Persistently depressed patients had higher Rankin scores (Kruskall Wallis $\mathrm{X}^{2}=11.58, \mathrm{df}=3, \mathrm{p}=0.009$ ). Recurrent cases were older than transient cases and persistent cases $(73.4,64.1$, and 63.1 years respectively), albeit not significantly $(F=2.49, d f=3, p=0.06)$. Non-depressed patients more often were highly educated $\left(X^{2}=8.00, d f=3, p=0.04\right)$. Nondepressed patients had lower neuroticism scores than recurrent cases $(F=4.5$, $d f=3, p=0.004)$. There were no differences in severity of depression, location of the lesion, cognition, and sex. Recurrent cases were prescribed antidepressants more often albeit not significantly. There was no significant correlation between cognitive and functional deficits and persistent, transient or recurrent depression. Four patients received an intravenous thrombolytic treatment. These patients were distributed equally among the non-depressed, incidentally, persistently and recurrently depressed patient groups.

Table 4. Demographic variables of early and late PSD patients.

\begin{tabular}{lll}
\hline & Early (N=26) & Late (N=24) \\
\hline Sex (v) & $51.9 \%$ & $30.4 \%$ \\
Age (mean, SD) & $66.2(12.2)$ & $66.3(11.8)$ \\
Education (high) & $14.8 \%$ & $39.1 \% *$ \\
MMSE (median, range) & $26.0(19-29)$ & $27.0(18-30)$ \\
Barthel (median, range) & $18.0(0-20)$ & $20(0-20)$ \\
Rankin (mean, SD) & $2.74(1.1)$ & $2.13(1.1)$ \\
Hamilton score (mean, SD) & $16.7(7.0)$ & $8.8(5.4)^{*}$ \\
Major depression & $66.7 \%$ & $52.2 \%$ \\
Neuroticism mean (SD) & $33.2(6.3)$ & $32.6(7.4)$ \\
Cortical infarction & $51.9 \%$ & $34.8 \%$ \\
Left sided lesion & $40.7 \%$ & $47.8 \%$ \\
Antidepressants & $40.0 \%$ & $41.7 \%$ \\
History of depression & $56.5 \%$ & $28.6 \%$ \\
Family history of depression & $37.0 \%$ & $13.6 \%$ \\
\hline
\end{tabular}

${ }^{*} p<0.05$

Table 4 shows the characteristics of patients diagnosed as depressed at the first follow-up visit or later. Patients suffering from late-onset PSD were more highly educated $\left(X^{2}=3.82, d f=1, p=0.05\right)$. They more often were men albeit not significantly. Early and late PSD patients did not differ in level of neuroticism. 


\section{Discussion}

In this study of PSD in first-ever stroke patients during a one year prospective follow-up, we examined the persistency of PSD. The cumulative incidence of PSD was $36.2 \%$. Half of these patients were diagnosed as depressed within 1 month after stroke and incidence rates declined after the acute phase after stroke. The prevalence rates at subsequent follow-up times initially tended to decrease and at 6 months to increase but all in all remained comparable to baseline throughout the follow-up period. In approximately $30 \%$ of patients who were depressed within the first three months the duration of disease was restricted to 1 follow-up visit. In $44 \%$ of these patients, PSD recurred within the first year after stroke. Patients in whom disease recurred were older than patients in whom disease was either restricted to 1 visit or persisted over a longer period. Patients with persistent symptoms of PSD more often met criteria for an episode of major depression somewhere in their disease course. This is in accordance with the findings of Morris et al. [141]. Moreover, these patients were more severely handicapped than patients with a transient disease episode. In our study, nondepressed patients were more often highly educated (44\%), whereas $7.7 \%$ of patients in whom disease persisted were highly educated. Also, patients with a late onset of PSD were more often highly educated compared to patients with an early onset of PSD.

In the literature, PSD is often studied in longitudinal prevalence studies of several years with long time periods between follow-up visits [28]. A strong point of our study is that patients were examined every 3 months, providing insight into incident depressive cases and the dynamic character of depression in the course of PSD during the first year. In a longitudinal study performed by Astrom et al., in 80 stroke patients followed over 3 years the investigators found a prevalence of $25 \%$ at the acute stage, and that $60 \%$ of these patients recovered within 1 year [29]. Berg et al. found that $54 \%$ of 100 stroke patients were at least mildly depressed during an 18 month follow-up and approximately half of the patients with depressive symptoms within the first 2 months also had depressive symptoms after 12 and 18 months [30]. In our study we found that most patients diagnosed with PSD in the acute stage recovered. However, in our study patients were screened for the DSM-IV diagnosis of minor and major depression. Thus our study comprised only the more severely affected patients and patients with mild depressive symptoms were excluded. It is conceivable that the incidence of patients with persistent depressive symptoms is actually higher. Two studies 
reported that patients with less severe depression suffered longer [28, 142]. Moreover, in our study PSD recurred in $28 \%$ of patients. Patients with recurrence of disease were older. There was no difference in frequency of antidepressive drug treatment between patients who recovered or in whom disease persisted or recurred.

Thus far the causality of PSD has not been fully elucidated. Although the literature reports no association between the vulnerability for PSD and level of education we found that PSD patients were less often highly educated than non-depressed stroke patients [133]. Furthermore patients suffering from late onset PSD were often more highly educated than patients suffering from early onset PSD. However, due to small numbers, a conclusion should be taken with caution. Biopsychosocial and social factors all account for the occurrence of PSD at all stages in stroke rehabilitation [25, 132, 133, 143]. Some authors report a time-dependent causality [118]. In the early disease stage especially the course of symptoms is dynamic. Whereas most recovery is seen in the first weeks after stroke this is also the period in which the incidence of PSD is highest [144, 145]. Tateno et al reported a time-related change in symptomatology and concluded that early depressive symptoms are related to biological causality provoked by the brain damage itself, whereas in late onset symptoms social and psychological markers may attribute more importantly to the cause of depression [118]. Berg et al. found that early symptoms were associated with older age and late onset symptoms were associated with stroke severity and male sex [30]. Astrom found that in the early stage, dependence in activities of daily living predicted PSD, whereas in the long term having few social contacts was more predictive [29].

Our study has some limitations. First of all since we had a limited follow-up period of one year we cannot be specific about the persistency of PSD in patients who were diagnosed depressed at follow-up visits later than the first 3 months. Secondly, we used screening instruments to identify patients at risk of suffering from PSD. Although our screening method was shown to be highly sensitive, some patients with (major or minor) depression using the SCID-I on the previous measurement, may falsely have scored below threshold on the next screening since patients were dichotomized depressed or non-depressed [76]. It is conceivable that analysis of the severity of depression on a continuous scale would have increased the sensitivity of our methods. Small numbers limited the detection of significant relationships between the diagnosis of depression and demographic variables, especially between subgroups. A study into the causality and risk factors for depression, however, would require a different study design 
and was beyond the scope of this study. Additionally, the prescription of antidepressant agents may have influenced the conclusions drawn about the persistency and recovery of PSD. However, there were no differences in the number of patients who were prescribed antidepressants in the disease course between persistent and recovered PSD patients.

Furthermore, since we excluded patients with most severe cognitive deficits and severe aphasia or somatic morbidity we cannot rule out bias. Since excluded patients were not assessed we cannot determine how this affected our results. However, it is conceivable that patients with more handicap and cognitive deficits more often suffer from depression and that the incidence of PSD in our study is actually higher. The informed consent warranted that patients would understand the procedure. This generally applies to studies warranting a long term cooperation. In a previous study we reported that patients with lower MMSE scores experienced more problems fulfilling the rating scales and had higher dropout rates [76].The course of depression in such patients may differ from those studied. In literature these patients often are excluded due to the lack of sufficient diagnostic measures. Nowadays, there are screening measurements to define the severity of depression in aphasic patients [16].More insight into PSD in these patients is of great necessity. It is important to know how cognitive and functional deficits influence the course of PSD. In our study patients who did not exceed the cut-off scores of the attributed screening measures for depression were not assessed. Therefore, we cannot determine how these deficits evolve over time and how they influenced our results over time. However, there were no significant correlations between baseline cognitive or functional deficits and the subgroups of PSD in our study. Finally, we had a substantial amount of drop-outs. These patients however did not differ in frequency or severity of depressive symptoms so it is not likely that this affected our results.

A better understanding of the spontaneous course of PSD may assist in deciding if and when a patient should be treated with antidepressants or non-pharmacological interventions.

To date there is little evidence to support routine use of antidepressants in the prevention and treatment of PSD, only a few studies report a beneficial effect of antidepressants on depressive symptoms and stroke outcome [31, 100,137].

In our study, we found that PSD is most frequent in the acute phase after stroke. Prevalence rates remained more or less stable throughout the first year after stroke. Most patients recovered spontaneously within 3 months. Since a treatment effect of antidepressants is not obvious until weeks after initiation, drug treatment 
will not be attributive to recovery. So, in most cases it will be acceptable to wait and see. However, in a substantial number of patients depression recurred. These patients were older. Furthermore, chronic diseased patients more often suffered from major depression. These patients may benefit from antidepressants and nonpharmaceutical interventions if symptoms persist for 3 months. More research into the treatment of PSD is warranted. 



\section{Chapter \\ Depressive symptoms and executive functioning in stroke patients. A follow-up study.}

Adapted from:

A. Bour, S. Rasquin, M. Limburg, F. Verhey. Depressive symptoms and executive functioning in stroke patients. A follow-up study. International Journal of Geriatric Psychiatry. In press. 


\section{Abstract}

Background: Cognitive and emotional sequellae are commonly observed in stroke patients and these symptoms often co-occur. Diagnosis can be difficult since symptoms of depression and executive dysfunction overlap.

Objective: To study the longitudinal relationship between depressive symptoms and executive dysfunction in stroke patients.

Methods: The study comprises of 116 first-ever stroke patients who were followed-up for two years and who were assessed for emotional and cognitive sequellae after 1, 6, 12, and 24 months. Emotional disturbances were evaluated using the SCL-90 depression subscale. Executive functions were assessed using compound scores of a combination of the interference scores of the Stroop Colour Word Test and the Concept Shifting Test.

Results: 25 patients suffered from both depressive symptoms and executive dysfunction, 28 patients were depressed with no signs of executive dysfunction, and 13 patients showed executive dysfunction with no depressive symptoms. Patients with executive dysfunction had higher mean SCL-90-D scores compared to patients with no executive dysfunction (30.9 (SD 11.7) versus 26.2 (SD 11.1, $p=0.037$ ). Depressive symptoms were predictive for executive dysfunction. After two years $66.6 \%$ and $53.3 \%$ of patients with both depressive symptoms and executive dysfunction at baseline still had depressive symptoms and executive dysfunction respectively and had worse prognostic outcome than patients with depressive symptoms or executive dysfunction alone.

Conclusions: Symptoms of depression and executive dysfunction are highly prevalent in stroke patients and often co-occur. These patients are more at risk for poor stroke outcome, chronic depression and cognitive deterioration. 


\section{Introduction}

Stroke not only results in considerable physical impairments but often causes cognitive and emotional sequellae which also have a negative impact on stroke survival and recovery. The prevalence of cognitive impairment is increased in stroke patients compared to normal elderly individuals and $20 \%$ will become demented $[3,33,146]$. Depression is observed in $20-60 \%$ of stroke patients in the acute phase and in the long term $[2,5,6,147,148]$.

In the elderly depressive symptoms and cognitive impairment commonly co-occur $[149,150]$. Elderly suffering from depression may be more prone to developing mild cognitive impairment ( $\mathrm{MCl}$ ), which may be a pre-dementia state [151], and dementia, compared to healthy contemporaries, and tend to deteriorate cognitively more swiftly than non-depressed controls. Those suffering from chronic and mild depressive symptoms show more cognitive impairments than elderly who are more severely affected [149, 152]. Alternatively, depression is highly prevalent in the pre-dementia stages of Alzheimer's disease (AD) and particularly in vascular cognitive impairment (VCI) [149, 152-154]. Moreover, symptoms of impaired psychomotor speed and executive functioning are characteristic for depression in the elderly, especially in the presence of cerebrovascular risk factors [155-159].

In stroke patients symptoms of dysexecutive functioning and depression are highly prevalent and these patients have a worse stroke outcome [41, 160-163].The dysexecutive syndrome is characterized by disturbances in initiation, complex attention, and planning. A number of patients suffering from these symptoms may suffer from depression and may benefit from antidepressive treatments. This also applies to patients in whom both diagnoses prevail, since treatment of depression results in better cognitive performance in these patients [164, 165]. Others, however, will suffer from $\mathrm{VCl}$ and are possibly prone to become demented in time [149, 166].

This bidirectional relationship between symptoms of depressed mood and cognitive dysfunction is complex and not fully understood. According to the vascular depression hypothesis, depression in late life and dysexecutive functioning co-occur since they both evolve from frontostriatal dysfunction caused by vascular lesions in the frontal lobes and basal ganglia [21, 157, 167-169]. Vataja et al. described this depression-executive dysfunction in stroke patients and found this to be related to damage to the frontal subcortical circuits. These patients had more severe depressive symptoms and more difficulties in daily living [22]. 
The aim of this study was to study the temporal relationship between depressive symptoms and dysexecutive dysfunction in stroke patients. This is important in order to better understand the clinical and physiological relationship between these entities and to develop improved treatment strategies.

\section{Methods}

\section{Patients}

Patients included in this study were selected from CODAS and comprised of consecutive first-ever supratentorial stroke patients who visited the emergency department between January 2000 and August 2001. Study design and participants of CODAS have previously been described in more detail [80]. All participants gave informed consent and the study was approved by the local medical ethics committee of the Maastricht University.

In short, patients were asked to participate within 48 hours after stroke and were administered a clinical and complete neuropsychological examination at $1,6,12$, and 24 months after stroke.

All participants underwent a structured medical and neurological assessment including medical history, physical examination, laboratory studies, and brain CT scan. Stroke was diagnosed by a neurologist by clinical evaluation and a brain CT scan. CT was performed on the day of admission or the day after. These data were registered in the Maastricht Stroke Register (MSR). Location of the symptomatic lesion was determined on clinical syndrome and CT-scan information. Two neurologists blinded for neurological signs examined the scans independently ( $\mathrm{k}$ of 0.69). Brain atrophy was scored in a semi-quantitative way as none, mild, moderate, and severe by comparison of the CT-scans of the study participants to four control scans which were selected by experts [78].

Inclusion criteria were a first hemispheral stroke, age over 40 years, adequate post-stroke language abilities in Dutch, and an initial post-stroke MMSE score $>15$. Exclusion criteria were severe aphasia, pre-stroke dementia, and other major neurological or psychiatric disorders that could interfere with the neuropsychological testing. 


\section{Neuropsychological assessment}

At $1,6,12$, and 24 months after stroke, patients were administered a complete neuropsychological test battery including the MMSE [50] and CAMCOG [170, 171]. Compound scores for executive functioning were calculated from a combination of the interference score of the Stroop Colour Word Test (SCWT) [172] and the interference score of the Concept Shifting Test (CST) [173]. Patient scores were converted to standardized scores with the formula: $Z=$ (patient score-norm score) / SD of the norm group. The interference scores of the Stroop and the CST were defined by the following formula: Part III-mean (Part I and Part II). Cognitive functioning was compared to that of a norm group of healthy elderly inhabitants embedded in the Maastricht Aging study (MAAS). Norm tables are stratified according to age, sex, and level of education. A score lower than the $10^{\text {th }}$ percentile of the z-scores of the norm group defined a deficit in executive functioning. Higher compound scores for executive functions indicates worse performance.

Symptoms of depression were assessed at each follow-up using the depression subscale of the Symptom Check List (SCL-90-D) [70] . The SCL-90 is a self-rating scale comprising a wide range of psychological complaints. Patients were asked to indicate on a 5-point scale how much hindrance they experienced from psychiatric complaints in the last week. The validity of the SCL-90-D for diagnosing depression in stroke patients was demonstrated previously by our team [76]. A cut-off score of 24/25 was administered.

Functions in daily living were assessed by the Interview for Deterioration in Daily life in Dementia (IDDD) [83], which was completed by the caregivers. The IDDD assesses self-care and complex activities, with attention being paid to lack of initiative and impaired performance. The amount of initiative the patient still shows and the amount of help needed are rated on a 4-points scale.

Pre-stroke cognitive functioning was assessed by means of a semi-structured interview based on the DSM-IV diagnostic criteria for dementia with a patients' caregiver. The caregiver was asked whether the patient had memory problems or other cognitive impairments before stroke and whether these impairments interfered with daily living.

Diagnosis of dementia was based on data including information about medical history, test performance, structured information from a patient's informant about daily life functioning, and clinical observation. Two experienced clinicians independently made the diagnosis according to the DSM-IV criteria for dementia, blind to MMSE data. The diagnosis of VaD was based on the NINDS-AIREN criteria. 
Agreement between the two raters was $k=0.88 ; p=0.01$. If there was a discrepancy in diagnosis a consensus meeting was arranged. If consensus was not reached, a patient was not considered to be demented.

\section{Statistical Analyses}

Statistical analyses were performed with the Statistical Package for Social Sciences version 16 (SPSS-16). For the comparison of characteristics between patients who completed the follow-up and who did not complete the follow-up Students' t-tests were performed in case of longitudinal variables. For dichotomous variables $\mathrm{X}^{2}$ tests were performed. For further analyses only patients with complete results for all assessments were analyzed.

To measure the group variance between patients with no depressive symptoms or dysexecutive functioning (ND-NE), patients with only depressive symptoms (D-NE), patients with only dysexecutive functioning (ND-E) and patients with both depressive symptoms and dysexecutive functioning (D-E) multi comparison ANOVA was performed with post-hoc Bonferroni correction. For dichotomous variables $\mathrm{X}^{2}$ tests were performed.

For cross-sectional analyses of depression in patients with and without executive dysfunction mean scores on the SCL-90 depression subscale were compared. Repeated measurements ANOVA was performed to compare mean scores between follow-ups.

To study whether the SCL-90 predicted executive functioning a multiple regression analysis was performed, with executive functioning as dependent variable, and the SCL-90 depression subscale score, age, level of education, and sex as independent variables. To study whether the compound score for executive functioning predicted depressive symptoms a multiple regression analysis was performed with the SCL-90 depression subscale score above the cut-off score as dependent variable and the compound score for executive functioningage, level of education, and sex as independent variables. A p-value of $\leq 0.05$ was defined as statistically significant.

\section{Results}

One hundred and forty patients completed the two year follow-up period. Table 1 presents the patient characteristics of the patients who completed the follow-up 
and who did not. After two years 27 patients had died, 24 refused further participation, 3 were too ill, and 2 were untraceable.

Table 1. Demographic variables at baseline.

\begin{tabular}{llll}
\hline & $\begin{array}{l}\text { Complete follow-up } \\
(\mathrm{N}=140)\end{array}$ & $\begin{array}{l}\text { No complete follow- } \\
\text { up (N=54) }\end{array}$ & \\
\hline Age (SD) & $65.8(12.0)$ & $74.6(11.5)$ & 0.000 \\
Sex (F) \% & 45.7 & 42.6 & \\
Education \% & $50.7 / 37.1 / 12.1$ & $70.4 / 16.7 / 13.0$ & 0.019 \\
MMSE (SD) & $26.0(3.3)$ & $24.1(3.7)$ & 0.001 \\
SCL-D score (SD) & $28.0(11.8)$ & $28.5(13.8)$ & \\
Rankin (SD) & $3.0(1.34)$ & $3.2(1.42)$ & 0.022 \\
Diagnosis dementia \% & 7.9 & 20.4 & 0.021 \\
IDDD-initiative (SD) & $26.7(9.9)$ & $22.6(11.3)$ & \\
IDDD-help (SD) & $9.4(12.4)$ & $13.5(14.2)$ & \\
Left sided lesion \% & 39.6 & 52.8 & \\
Lacunar lesion \% & 61.8 & 50.0 & \\
Pre-existent asymptomatic vascular lesions \% & 32.1 & 35.4 & \\
Leukoaraiosis \% & 19.4 & 35.4 & \\
Brain atrophy \% & 65.0 & 80.4 & \\
Diabetes Mellitus \% & 14.0 & 16.7 & \\
Hypertension \% & 38.3 & 35.8 & \\
Ischemic heart disease \% & 25.0 & 30.2 & \\
Executive dysfunction \% & 35.4 & 41.0 & \\
Score above SCL-D cut off \% & 40.0 & 24.1 & \\
\hline
\end{tabular}

Educational level: low = primary school / junior vocational education, medium = senior vocational education, high $=$ Higher professional education / university. MMSE: Mini Mental State Examination (minimum score: 0, maximum score: 30). SCL-D: 90-items Symptom Checklist, depression subscale (minimum score 16, maximum score: 80, cut-off score 24 / 25). Rankin (no handicaps: 0, bedridden: 5). IDDD-initiative: Interview for Deterioration in Daily Living activities in Dementia, initiative subscale (minimum score: 0, maximum score: 36). IDDD-h: Interview for Deterioration in Daily Living activities in Dementia, help subscale (minimum score: 0 , maximum score: 44 ).

Patients who did not complete the follow-up were older, lower educated, had lower MMSE scores at baseline and more often exceeded the cut-off score of the SCL-90 depression scale compared to patients who completed the follow-up.

At baseline we had complete test results on both the SCL-90 and the executive functioning of 116 patients. The 24 patients for which we had incomplete data did not differ in age, MMSE-score or level of handicap to those patients who completed all tests. Of these 116 patients, twenty five (22\%) exceeded the SCL-90 depression scale cut-off and scored above the $10^{\text {th }}$ percentile of the norm group for executive dysfunctioning. Twenty eight patients (24\%) showed depressive symptoms but had no signs of executive dysfunction whereas thirteen patients 


\section{Chapter 5}

(11\%) showed executive dysfunctioning without depressive symptoms and fifty patients (43\%) had neither depressive symptoms nor executive dysfunctioning (Table 2). Table 2 shows the baseline patient characteristics of these four patient groups.

Table 2. Baseline characteristics of patients without depressive symptoms or executive dysfunction, with depressive symptoms without executive dysfunction, without depressive symptoms with executive dysfunction, with depressive symptoms and executive dysfunction

\begin{tabular}{lllll}
\hline & ND-NE (n=50) & D-NE (n=28) & ND-E (n=13) & D-E (n=25) \\
\hline Age (SD) & $65.1(10.8)$ & $62.9(12.2)$ & $66.2(11.6)$ & $69.5(13.8)$ \\
Sex (F) \% & 36.0 & 50.0 & 30.8 & 52.0 \\
Education (low/medium/ high) \% & $46.0 / 32.10 / 22.0$ & $53.6 / 42.9 / 3.6$ & $46.2 / 53.8 / 0.0$ & $48.0 / 40.0 / 12.0$ \\
MMSE (SD) & $27.0(3.1)$ & $26.8(2.8)$ & $26.4(2.8)$ & $24.0(3.8)^{*}$ \\
SCL-D score (SD) & $19.8(2.3)$ & $37.5(11.7)^{*}$ & $19.8(2.5)$ & $36.6(10.4)$ \\
Rankin (SD) & $2.6(1.5)$ & $3.2(1.2)$ & $3.3(1.3)$ & $3.3(1.3)$ \\
Diagnosis dementia \% & 2.0 & 7.1 & 0.0 & $24.0 \#$ \\
IDDD-initiative (SD) & $30.2(7.8)$ & $25.2(10.7)$ & $26.1(8.7)$ & $24.8(10.8)$ \\
IDDD-help (SD) & $4.5(8.6)^{*}$ & $12.0(14.1)$ & $5.6(10.4)$ & $13.5(13.1)$ \\
Left sided lesion \% & 42.9 & 29.6 & 41.7 & 38.9 \\
Lacunar lesion \% & 61.9 & 64.0 & 58.3 & 63.6 \\
Frontal region or basal ganglia & 37.5 & 42.3 & 46.2 & 32.0 \\
$\quad$ lesion \% & & & & \\
Pre-existent asymptomatic vascular & 23.4 & 25.9 & 30.8 & 39.1 \\
$\quad$ lesions \% & & & & \\
Leukoaraiosis \% & 10.6 & 25.9 & 7.7 & 17.4 \\
Diabetes Mellitus \% & 6.1 & 7.4 & 30.8 & 12.5 \\
Hypertension \% & 39.6 & 42.3 & 33.3 & 37.5 \\
Ischemic heart disease \% & 20.4 & 22.2 & 53.8 & 25.0 \\
Brain atrophy \% & 74.4 & 48.0 & 69.2 & 71.4 \\
\hline
\end{tabular}

Comparison of group variances between groups using one-way ANOVA with post-hoc Bonferroni corrections. For dichotomous variables Chi-square tests are attributed. ${ }^{*} \mathrm{p}<0.05, \# \mathrm{X} 2=12.6, \mathrm{df}=3$, $\mathrm{p}=0.005$. Educational level: low $=$ primary school $/$ junior vocational education, medium $=$ senior vocational education, high $=$ Higher professional education / university. MMSE: Mini Mental State Examination (minimum score: 0, maximum score: 30). SCL-D: 90-items Symptom Checklist, depression subscale (minimum score 16, maximum score: 80, cut-off score: 24 / 25). Rankin (no handicaps: 0 , bedridden: 5). IDDD-initiative: Interview for Deterioration in Daily Living activities in Dementia, initiative subscale (minimum score: 0 , maximum score: 36 ). IDDD-h: Interview for Deterioration in Daily Living activities in Dementia, help subscale (minimum score: 0 , maximum score: 44 ).

Patients in the D-E group had lower MMSE scores on average (24.0 (SD 3.8) than patients in the D-NE group (26.8 (SD 2.8), $p=0.01$ ) and patients in the ND-NE group (27.0 (SD 3.1), $p=0.001$ ). Moreover, patients in the $D-E$ group were more often demented $(24.0 \%$ versus $0.0 \%, 7.1 \%$, and $2.0 \%$ in the ND-E, D-NE and ND-NE groups respectively, $\left.\mathrm{X}^{2}=12.6, \mathrm{df}=3, \mathrm{p}=0.005\right)$. Patients in the $\mathrm{D}-\mathrm{E}$ and patients in 
the D-NE groups had higher scores on the IDDD-help measurements than patients in the ND-NE group (13.5 (SD 13.1), 12.0 (SD 14.1) versus 4.5 (SD 8.6) respectively, $p=0.009$ and $p=0.037)$.

Patients with executive dysfunction were older, had lower scores on the MMSE and Camcog, were more often demented, were more often depressed, and had more difficulties in daily life than patients without executive dysfunction. They more often had diabetes mellitus compared to patients without executive dysfunctions $\left(20.0 \%\right.$ and $\left.7.3 \%, X^{2}=4.8, d f=1, p=0.04\right)$. There were no differences in amount of pre-existent vascular brain damage or lesion location. Patients with executive dysfunction had higher mean SCL-90-D scores compared to patients with no executive dysfunction (30.9 (SD 11.7) versus 26.2 (SD 11.1, $p=0.037$ ) and more often scored above the cut-off score of the SCL-90-D (65.8\% versus $35.9 \%, X^{2}=9.2$, $d f=1, p=0.002)$. After correction for age, gender, and education level this difference was still significant.

Patients with depressive symptoms had lower scores on the MMSE and Camcog, were more often demented, more often had executive dysfunction, performed worse in activities of daily living compared to non-depressed patients. Depressed patients did not differ in vascular risk factors, pre-existent vascular brain damage or lesion location compared to non-depressed patients. Depressed patients more often showed executive dysfunction compared to non-depressed patients $(47.2 \%$ versus $20.6 \%, X^{2}=9.2, d f=1, p=0.002$ ). However, after correction for age, gender, and education level compound scores for executive dysfunction did not significantly predict depression.

Figure 1 shows the cross-sectional mean scores on the SCL-90 depression subscale at 1 and 2 years after the event of the depressed patients with and without executive dysfunction. 


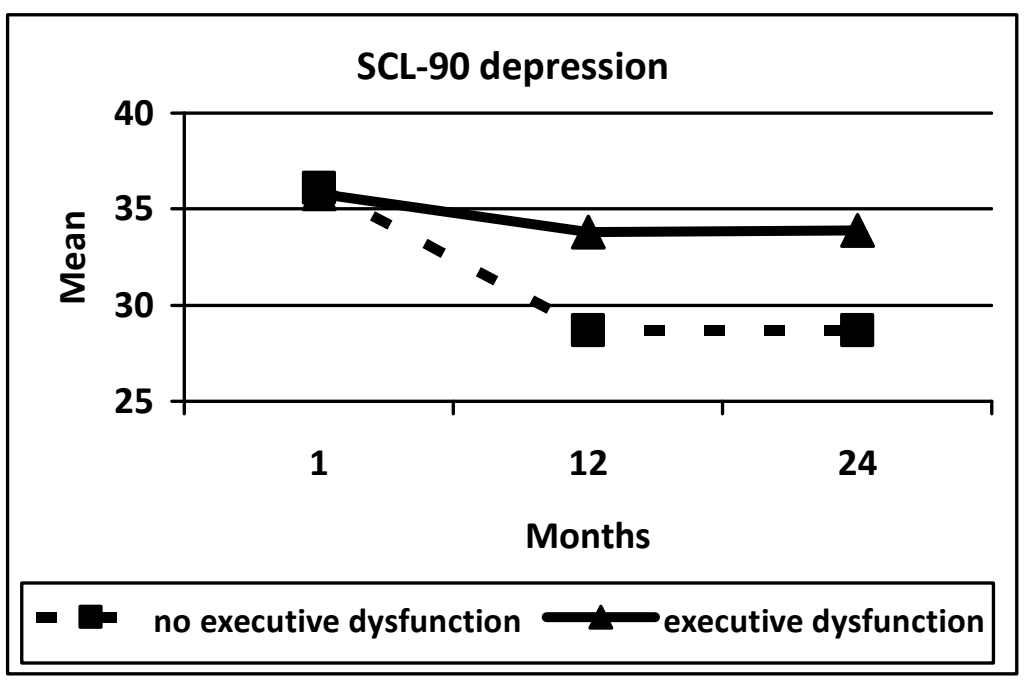

Fig. 1. Mean score on the SCL-90 depression subscale for depressed patients with and without executive dysfunction

At baseline and later assessments cross-sectional SCL-90 scores of depressed patients with executive dysfunction did not differ significantly from patients without executive dysfunction. In patients without executive dysfunction however SCL-90 depression scores improved significantly after 12 months compared to baseline. In patients with executive dysfunction SCL-90 depression scores did not change significantly.

Table 3. Follow-up of patients per baseline diagnostic category (ND-NE, D-NE, ND-E, and D-E).

\begin{tabular}{llllll}
\hline \multicolumn{5}{c}{ Diagnosis at 24 months follow-up } & \\
& & ND-NE & D-NE & ND-E & D-E \\
\hline Diagnosis at & ND-NE $(n=50)$ & $79.2 \%$ & $18.8 \%$ & $2.1 \%$ & $0.0 \%$ \\
baseline & D-NE $(n=28)$ & $36.4 \%$ & $40.9 \%$ & $13.6 \%$ & $9.1 \%$ \\
& ND-E $(n=13)$ & $25.0 \%$ & $25.0 \%$ & $50.0 \%$ & $0.0 \%$ \\
& D-E $(n=25)$ & $13.3 \%$ & $33.3 \%$ & $20.0 \%$ & $33.3 \%$ \\
\hline
\end{tabular}

Table represents the percentages of patients diagnosed as ND-NE, D-NE, ND-E, and D-E after 24 months per baseline diagnostic category.

$X^{2}=48.0, d f=9, p<0.000$

Table 3 shows the follow-up of patients per baseline category after two years. Of the patients in the D-E group $13.3 \%$ recovered completely whereas $33.3 \%$ still had depressive symptoms and a dysexecutive syndrome. Fifty percent of patients in 
the D-NE group and $67 \%$ of patients in the D-E group still suffered from depression after two years. After two years $50 \%$ of the ND-E group and $53 \%$ of the D-E group still showed executive dysfunction.

\section{Discussion}

In this study we examined the relationship between depressive symptoms and the dysexecutive syndrome in first-ever stroke patients. In accordance with previously reported findings by Vataja et al. 4 groups of patients could be distinguished: Patients with no neuropsychological or emotional complaints, patients suffering from depressive symptoms, patients suffering from executive dysfunction, and patients suffering from both depressive symptoms and executive dysfunction [22]. We found that depressive symptoms and executive dysfunctioning were highly prevalent and co-occurred in $22 \%$ of patients 1 month after stroke. Patients suffering from depression and executive dysfunction had lower MMSE scores and were more often demented. Vataja et al. found that patients with the depressionexecutive dysfunctioning syndrome after stroke had more severe depressive symptoms and performed worse in daily living [22]. In our cohort these characteristics were comparable between the subgroups. Patients suffering from executive dysfunction had higher scores on the SCL-90 depression subscale and more often had diabetes mellitus. Patients with depression had higher compound scores for executive functioning than patients without depression. In regression analyses after correction for age, sex, and diabetes mellitus, depressive symptoms as measured by the SCL-90 predicted executive dysfunction. Executive dysfunctioning did not predict depression in a regression analysis. This is in line with findings reported by Cui et al. in geriatric depression [174]. A previous study among patients with post-stroke depression reported deficits in memory, attention, problem solving and mental speed [147]. Other studies reported that cognitive deficits and executive dysfunction are risk factors for depression [169, 175] or found no relationship [157]. Pohjasvaara et al. studied patients with dysexecutive syndrome after stroke and found higher Beck Depression Inventory scores in patients with the dysexecutive syndrome but no higher incidence of depression [102]. SCL-90 scores improved in depressed patients without executive dysfunction compared to patients with executive dysfunction. After two years $33 \%$ of patients suffering from depressive symptoms and executive dysfunction still 
suffered from these sequellae. Furthermore, $57 \%$ of the depressed patients and $50 \%$ of the patients with executive dysfunction had not recovered after two years. The relationship between cognitive impairment and depression after stroke in the elderly in general is well established and literature reports bidirectional bonding $[147,153,168,176]$. Symptoms of mental slowing, lack of initiative, difficulties in planning and decision making overlap [158, 177, 178]. The relationship between executive dysfunction and depression in stroke patients is recognized but most studies focus on global cognitive functioning. In late life depression and poststroke depression symptoms of executive dysfunction may result from damage to the frontal subcortical circuits according to the vascular depression hypothesis comparable to vascular cognitive impairment $[167,179]$. This theory is supported by studies that found an association between executive dysfunctioning in depression and frontally located lesions $[22,162,179]$. These studies state that the depression-executive syndrome is a distinguishable entity in vascular affected patients. Although symptoms show a vast similarity it is possible to distinguish depression from a dysexecutive syndrome $[102,161,168,180]$. This is important since these entities differ in prognosis and therapeutic interventions [22, 181, 182]. Hama et al. studied the functional outcome of stroke patients with depression or apathy and found that especially the latter predicted worse functional outcome and interfered with stroke recovery [183]. Another study among geriatric patients found that patients with executive dysfunction had a worse quality of life [157]. We found that patients with both depression and executive dysfunction had more difficulties in daily living, albeit not significantly. Depressive symptoms in patients with vascular dementia are highly prevalent and more persistent than depressive symptoms in subjects with Alzheimer's disease and mild cognitive impairment and react less well to medication. Furthermore, depressive symptoms in vascular compromised brains can result in dementia [149, $166,184]$. A follow-up study in stroke patients reported that depression especially in the acute stage predicted cognitive decline. Patients who remained depressed and patients with both depressive and cognitive disturbances had worse stroke outcomes [162]. Narushima et al. found that cognitive deficits were reversible in patients in whom depressive symptoms recovered [180]. Alternatively, Saxena et al. found that functional recovery was more swift after treatment of depression post-stroke, but that the level of outcome was determined by neurological and cognitive deficits [185].

Despite our finding that depressive symptoms and executive dysfunction in stroke share demographic characteristics and depressive symptoms predicted executive 
dysfunction, we support the idea of distinguishing separate disease entities in stroke patients with symptoms of executive dysfunction. Patients suffering from both depression and executive dysfunction showed worse cognitive performance and more dementia than patients with depression or executive dysfunction alone. Moreover, we found less recovery from symptoms after two years in patients with depressive symptoms and executive dysfunction than in patients with depression or executive dysfunction alone.

A strong point of this study is that it is one of the few studies that distinguishes executive dysfunction from depression and that symptoms are followed-up crosssectionally over two years. There are some limitations which need mentioning. Our selection criteria required the exclusion of patients with severe cognitive deficits and patients with severe aphasia. Our results may not be referable to the cognitively severely impaired group of stroke patients. Furthermore, patients who did not complete the follow-up were older and lower educated than patients who completed the follow-up. Since age is related to executive dysfunction, the incidence of patients with executive dysfunction with or without depressive symptoms is possibly higher [186]. We used a self-rating scale to screen for depressive symptoms and did not diagnose depression by psychiatric clinical assessment. The SCL-90 is a questionnaire to assess psychiatric symptoms. In the screening for depression in stroke patients however, the SCL-90 is found to be a valid tool for the diagnosis of depression [76]. In addition, this enabled us to study the relationship between depressive symptoms and executive dysfunction in relatively mildly affected patients. It has to be mentioned that we did not dispose of information about the use of antidepressant agents. The effectiveness of antidepressant agents in these patients is yet to be explored more extensively. Nevertheless, it would be interesting to know how symptoms of depression and executive dysfunctions are influenced by antidepressant drugs. It is conceivable and that the incidence of depression in our population is higher and that our results are weakened. Neuroradiological information is based on CT-scan findings as study data were collected several years ago $[78,80]$. Location of the lesion was determined by the CT-image and a patients clinical presentation. We found no relationship between lesion location, the presence of pre-existing nonsymptomatic vascular brain damage or atrophy and depression or executive dysfunctioning. Studying such potential effects however, would require an other study design including brain MRI which is more sensitive. Therefore, our results must be interpreted with caution. In this study we focused on the clinical 


\section{Chapter 5}

presentation of symptoms and their temporal evolvement and explaining the pathophysiology and causes was beyond our aims.

In this study we examined depressive symptoms and executive dysfunction in stroke patients and found that these entities often co-occurred. Furthermore, depressive symptoms predicted executive dysfunction. Symptoms of depression and executive dysfunction remained highly prevalent after two years. Patients who suffer from both depression and executive dysfunction are most at risk for chronic psychiatric and neuropsychological sequellae and a poor stroke outcome. Further research is needed to examine if appropriate treatment may alter the prognosis and improve outcome. 


\section{How predictive is the MMSE for cognitive performance after stroke?}

Adapted from :

A. Bour, S. Rasquin, A. Boreas, M. Limburg, F. Verhey. How predictive is the MMSE for cognitive performance after stroke? Journal of Neurology. 2010 April: 257(4): 630-637 


\section{Abstract}

Background: Cognitive deficits are commonly observed in stroke patients. Neuropsychological testing is time-consuming and not easy to administer after hospital discharge. Standardized screening measures are desirable. The Mini Mental State Examination (MMSE) is the most applied test to screen for cognitive deficits. Despite its broad use, predictive characteristics after stroke have not been investigated exhaustively.

Purpose: To define whether the MMSE is able to adequately screen for cognitive impairment and dementia after stroke and whether or not the MMSE can predict further deterioration or recovery in cognitive function over time.

Methods: One-hundred and ninety-four first-ever stroke patients without prestroke cognitive deterioration underwent an MMSE and a neuropsychological test battery at 1, 6, 12, and 24 months after stroke.

Results: The MMSE score 1 month after stroke predicted cognitive functioning at later follow-up visits. It could not predict deterioration or improvement in cognitive functioning in time. The cut-off score in the screening for 1 cognitive disturbed domain was $27 / 28$ with a sensitivity of 0.72 . The cut-off score in the screening for at least 4 impaired domains and dementia were 26 / 27 and 23 / 24 with a sensitivity of 0.82 and 0.96 respectively.

Conclusion: The MMSE has modest qualities in screening for mild cognitive disturbances and is adequate in screening for moderate cognitive deficits or dementia in stroke patients 1 month after stroke. Poor performance on the MMSE is predictive for cognitive impairment in the long term. However, it cannot be used to predict further cognitive deterioration or improvement over time. 


\section{Introduction}

Over half of stroke patients suffer from disturbances in cognition such as problems in memory and mental slowness. In the acute phase after stroke these cognitive deficits often remain unrecognised because at that time a patient frequently is not fully aware of possible cognitive dysfunction. Furthermore, a thorough neuropsychological evaluation is not routinely administered. After a longer period after stroke however, cognitive impairment is considerably prevalent $[3,5,187]$. These so called 'invisible' consequences can have a great negative impact on rehabilitation and outcome $[188,189]$.

Over the last decade, stroke care has become much more efficient, costs have lowered and hospital stay shortened. Most functional recovery occurs within the first months after stroke, and starting a rehabilitation course in an early stage is beneficial for a patient's outcome. Also cognitively impaired elders benefit from admission to an acute stroke and rehabilitation unit [190, 191]. Usually patients undergo physical and cognitive screening in an early stage to arrange the most effective rehabilitation program after hospital discharge, and to arrange appropriate home adjustments. Therefore, valid screening measurements are warranted as is screening for cognitive problems later in stroke recovery. At that time most stroke care often is provided by a general practitioner.

The Mini Mental Stage Examination (MMSE) is the most widely used instrument in screening for cognitive problems in hospitalised patients and in outpatient settings. It comprises thirty items providing information about orientation, attention, learning, calculation, delayed recall, and construction [50]. Several studies report an acceptable validity of the MMSE as a screening instrument and its relationship to functional outcome in stroke patients [53-55, 192]. Others conclude that it is not an accurate screening tool for cognitive deficits in stroke patients. It cannot differentiate between focal and diffuse lesions, it is language, age, and education dependent, it and is insensitive to right-sided lesions [40, 193, 194].

Although the value of the MMSE in the screening for cognitive dysfunction in stroke patients is still under debate, it is widely used and it has become a clinical standard. It is brief and easily applicable, and has a low interrater variability [194, 195].

The aims of this study were to see whether the MMSE is sufficiently accurate in the screening for mild and moderate cognitive disturbances and to determine whether it can be used in a two step approach, selecting patients with no cognitive 
deficits from those would need thorough cognitive testing, by a neuropsychological test battery. It is conceivable that patients with a poor score on the MMSE are prone to problems with rehabilitation and further deterioration and therefore would need closer follow-up. Therefore we studied whether the MMSE in an early phase could predict cognitive performance and cognitive deterioration over time.

\section{Materials and methods}

\section{Patients}

Between January 2000 and August 2001194 consecutive patients with a supratentorial stroke, admitted to the Neurology Department of the University Hospital Maastricht, were enrolled in this study. Study and patient characteristics have been described previously [42].Patients were asked to participate in the study within 48 hours after stroke.

All participants underwent a structured medical and neurological assessment including medical history, physical examination, laboratory studies, and brain CT. CT was performed on the day of admission or the day after. These data were registered in the Maastricht Stroke Register (MSR).

Stroke was diagnosed by a neurologist by clinical evaluation and a brain CT scan. Inclusion criteria were a first hemispheral stroke, age over 40 years, adequate post-stroke fluency in Dutch, and an initial post-stroke MMSE score $>15$. Exclusion criteria were severe aphasia, pre-stroke dementia, and other major neurological or psychiatric disorders, that could interfere with the neuropsychological testing.

Patients were followed-up 1, 6, 12, and 24 months after the event for clinical and neuropsychological evaluation.

The study was based on informed consent and was approved by the local medical ethics committee.

\section{Assessment of cognitive functioning}

Pre-stroke cognitive functioning was assessed by means of a semi-structured interview based on the DSM-IV diagnostic criteria for dementia with a patients' caregiver. The caregiver was asked whether the patient had memory problems or other cognitive impairments before stroke and how much these impairments 
interfered with daily living. This interview was repeated at each assessment. However, after the first assessment the focus shifted from pre-stroke functioning towards cognitive functioning compared to the previous assessment.

Within one month all participants were assessed by the standardised Dutch translation of the MMSE and a neuropsychological test battery by a well-trained neuropsychologist (SR) consisting of the following tests: CAMCOG, Concept Shifting Test, Stroop Colour Word Test, Auditory Verbal Learning test and the Groninger Intelligence Test. This battery assesses memory, language, mental speed, orientation, attention, praxis, executive functioning, calculation, and visuospatial functioning. Cognitive functioning was compared with that of a norm group from the Maastricht Aging Study (MAAS), which investigates normal cognitive ageing in healthy older volunteers. Norm tables are stratified according to age, sex, and educational level [196].

Table 1. Cognitive domains

\begin{tabular}{ll}
\hline Domain & Tests \\
\hline Memory & AVLT, total words direct recall and delayed recall \\
Mental speed & SCWT I, CST 0,I, and II \\
Executive functioning & Mean interference score SCWT, CST \\
Calculation & GIT, sums \\
Visuospatial & GIT, mental rotation \\
Orientation & CAMCOG items 139-148 (place, person, time) \\
Attention & Camcog items 178-179 (serial 7's, counting backward) \\
Praxis & Camcog items 183-186/188-189/191-193 (copying, ideational, ideomotor) \\
Reasoning & CAMCOG items 197-200, 201-203 (similarities, perception) \\
Language & CAMCOG items 149-163/181-182 (understanding, expression, writing) \\
\hline
\end{tabular}

AVLT: Auditory Verbal Learning Test / SCWT: Stroop Colour Word Test / CST: Concept Shifting Test / GIT: Groninger Intelligence Test

A score lower than the $10^{\text {th }}$ percentile of the score of the norm group defined a deficit on a cognitive domain [37]. Table 1 represents the ten cognitive domains tested. Patients were screened for disturbances in 1, 2, and at least 4 domains. These categories were chosen somewhat arbitrarily. Literature reports studies screening for at least 1 domain disturbed and in another study performed by Tatemichi et al. cognitive impairment was defined as a disturbance in at least 4 domains $[3,52,197]$.

Diagnosis of dementia was based on data including information about medical history, test performance, structured information from a patient's informant about daily life functioning, and clinical observation. Two experienced clinicians independently made the diagnosis according to the DSM-IV criteria for dementia, 
blind to MMSE data. The diagnosis of Vascular Dementia $(\mathrm{VaD})$ was based on the NINDS-AIREN criteria. Agreement between the two raters was $k=0.88 ; p=0.01$. If there was a discrepancy in diagnosis a consensus meeting was arranged. If consensus was not reached, a patient was not considered to be demented.

A change in cognitive performance was defined as an improvement or deterioration in the number of cognitive domains that were impaired.

\section{Statistical analyses}

For patient characteristics descriptive statistics were administered. To determine the prevalence of patients who improved or deteriorated cognitively during the follow-up period, only patients with complete follow-up data were studied.

Pearson's correlation coefficients were calculated to study a relationship between baseline MMSE-scores and MMSE-scores at later follow-up visits, and between baseline MMSE-scores and the number of cognitive domains disturbed. Spearman's Rho was calculated to study the relationship between baseline MMSEscore and the course of cognitive functioning at later follow-up visits.

Multivariate linear and logistic regression analyses adjusted for age, sex, and educational level were administered to see whether baseline MMSE-scores could predict a disturbance in cognitive domains tested, whether the MMSE could predict dementia, and whether baseline MMSE could predict the course of cognitive functioning at later follow-ups.

To assess a cut-off score of the MMSE in order to screen for impairment in at least one, two and four out of ten cognitive domains and to screen for dementia, Receiver Operating Characteristics (ROC) curves were obtained. The Area Under the Curve $(A \cup C)$ was calculated as a measure of the accuracy of the scale. The closer the value of $A U C$ is to 1,0 the more accurate a test is in determining impaired patients and non-impaired patients. Sensitivity, specificity, and the positive predictive value were calculated at the cut-off points.

Missing data were imputed by logistic regression. Data imputation was performed if there was another test covering the same cognitive domain available and if the missing test was administered previously or at a later follow-up.

All analyses were performed with the statistical Package for Social Sciences version 15 (SPSS-15). 


\section{Results}

Between January 2000 and June 2001194 first-ever stroke patients were included in this study. In this period, 592 stroke patients were diagnosed suffering from a stroke. Of these patients 80 died within 1 month after stroke and 363 were excluded ( 89 were not first-ever strokes, 57 had a stroke located in the brainstem or cerebellum, 46 MMSE <15, 34 had severe aphasia, 22 had comorbid neurological or psychiatric disorders, 6 were in a coma, 5 were not native Dutch speaking, 9 were younger than 40 years, 9 lived too far from the hospital, 6 were admitted too long after their stroke and 35 refused participation) [42].

Patient characteristics at baseline ( 1 month after the event) are presented in Table 2. During the follow-up 54 patients dropped out (27 died, 24 patients refused further participation, 1 patient was too ill at the 24 months follow-up, and 2 were untraceable.

Table 2. Demographic characteristics at baseline (1 month)

\begin{tabular}{lllll}
\hline & Total cohort & $\begin{array}{l}\text { Follow-up } \\
\text { completed }\end{array}$ & Drop outs & \\
\hline $\mathrm{N}$ & & & & $\mathrm{p}^{*}$ \\
Education (low) \% & 194 & 140 & 54 & \\
Sex (F) \% & 56.2 & 50.7 & 70.4 & 0.019 \\
Age mean (SD) & 44.8 & 45.7 & 42.6 & \\
Cortical lesion \% & $68.3(12.5)$ & $65.8(12)$ & $74.6(11.5)$ & $<0.000$ \\
Left sided lesion \% & 37.6 & 38.2 & 50.0 & \\
Rankin mean (SD) & 42.1 & 39.6 & 52.8 & \\
MMSE mean (SD) & $3.1(1.4)$ & $3.0(1.3)$ & $3.2(1.4)$ & \\
Diagnosis of dementia at baseline \% & $25.5(3.5)$ & $26(3.3)$ & $24.1(3.7)$ & 0.002 \\
\hline
\end{tabular}

* $\mathrm{p}$ values for significant differences in the comparison between patients who completed the follow-up and drop-outs are presented

SD: Standard Deviation

Rankin: no handicaps :0, bedridden: 5

MMSE: Mini Mental State Examination (minimum score: 0 , maximum score: 30 )

Drop outs were older than non drop outs (75.4 and 65.8 years respectively, $\mathrm{p}<0.001$ ), had lower baseline MMSE scores (24.1 and 26.0 respectively, $\mathrm{p}<0.001$ ), and were lower educated $(70.4 \%$ and $50.7 \%$ of patients were low educated respectively, $\mathrm{p}<0.001)$.

Table 3 shows the percentage of patients who deteriorated or improved at least in one domain of the cognitive domains tested compared to baseline at 12 and 24 
months follow-up. Patients were categorized into baseline MMSE scores over 27, between 27 and 24, or lower than 24 . Patients with MMSE scores below 24 were older than patients with scores higher than 27 (70.5 (SD 11.8) and 64.0 (SD 11.9) years respectively, $p=0.04)$ and more often low educated $(70 \%$ and $39.4 \%$ respectively, $\left.X^{2}=10.0, d f=4, p=0.04\right)$.

Table 3. Patients who improved or deteriorated in cognitive functioning according to baseline MMSEscore.

\begin{tabular}{lllll}
\hline & & Improved & No change & Deteriorated \\
\hline 12 months & Baseline MMSE $>=27$ & 40.8 & 40.8 & 18.4 \\
& $\begin{array}{l}\text { Baseline MMSE }<27 \text { and }>=24 \\
\text { Baseline MMSE }<24\end{array}$ & 65.6 & 18.8 & 15.6 \\
& & 58.6 & 10.3 & 31.0 \\
24 months & Baseline MMSE $>=27$ & 41.0 & 43.6 & 15.4 \\
& Baseline MMSE $<27$ and $>=24$ & 52.9 & 32.4 & 14.7 \\
& Baseline MMSE $<24$ & 51.7 & 17.2 & 31.0 \\
\hline
\end{tabular}

Numbers represent percentages.

Change in cognitive functioning means an improvement or deterioration of 1 in 10 previously described cognitive domains compared to baseline.

At 24 months follow-up the number of patients followed -up completely with baseline MMSE $>=27$ is $78, \mathrm{MMSE}<27$ and $>=24$ is 34 , and for MMSE $<24$ is 29

After 12 months most patients improved or remained stable in number of cognitive domains disturbed in each category of baseline MMSE score. Patients with baseline MMSE scores lower than 24 more often deteriorated. Patients with higher baseline MMSE scores more often were stable in the number of cognitive domains disturbed $\left(X^{2}=13.6, d f=4, p=0.009\right)$. After 24 months measures were comparable. Chi square tests between categories were not significant. There was no significant difference in mean baseline MMSE score, age, or educational level between patients who deteriorated cognitively during follow-up and patients who did not. These findings were comparable in patients with left-sided lesions and right-sided lesions and in demented and non-demented patients.

Baseline MMSE correlated well to scores on the MMSE at later follow-up visits 6, 12 , and 24 months: $r=0.77, r=0.76$, and $r=0.73$ respectively, $(p<0.01)$. The MMSE score at baseline related well to the number of cognitive domains disturbed at baseline and at later follow-up visits 6,12 , and 24 months: $r=-0.68, r=-0.70, r=-$ 0.62 , and $r=-0.69$ respectively $(p<0.01)$.

Table 4 shows the prevalence of patients suffering from at least 4 disturbances in cognitive domains and the prevalence of patients with a maximum of 1 disturbance based on their baseline MMSE score. 
To study whether baseline MMSE could predict the amount of disturbed cognitive domains multivariate regression analysis with adjustment for age, sex and level of education was performed. Baseline MMSE was a significant predictor for the amount of disturbed cognitive domains at baseline and later follow-up visits 6, 12, and 24 months after the event $(p<0.05)$. Furthermore, the MMSE was a significant predictor for the diagnosis of dementia in the multivariate model $(p<0.05)$.

Table 4. Percentages of patients with at least 4 cognitive domains and 1 or less domains disturbed.

\begin{tabular}{lllll}
\hline$\geq 4$ domains disturbed & & & & \\
& Baseline & 6 months & 12 months & 24 months \\
\hline Baseline MMSE & & & & \\
$<24$ & 80 & 80.0 & 75.9 & 79.3 \\
$24 \leq$ MMSE $<27$ & 53.1 & 28.1 & 25.0 & 29.0 \\
$\geq 27$ & 14.1 & 4.0 & 10.5 & 7.7 \\
\hline
\end{tabular}

\begin{tabular}{lllll}
\hline$\leq 1$ domains disturbed & & & & \\
& Baseline & 6 months & 12 months & 24 months \\
\hline Baseline MMSE & & & & \\
$<24$ & 6.7 & 3.3 & 17.2 & 10.3 \\
$24 \leq$ MMSE $<27$ & 9.4 & 25.0 & 46.9 & 29.0 \\
$\geq 27$ & 56.4 & 72.0 & 65.8 & 69.2 \\
\hline
\end{tabular}

At baseline the number of patients with a MMSE score $<24$ is 30, with a MMSE score between 24 and 27 is 32 , and with a MMSE score $\geq 27$ is 78 . Only patients with complete follow-up $(N=140)$ were studied.

Figure 1 shows the ROC of the MMSE in the screening for a disturbance in cognitive domains and for the diagnosis of dementia. At baseline, 163 patients suffered from at least 1 disturbed domain out of 10 cognitive domains, 137 patients from at least 2 and 85 patients suffered from at least 4 disturbed domains out of 10 cognitive domains. 


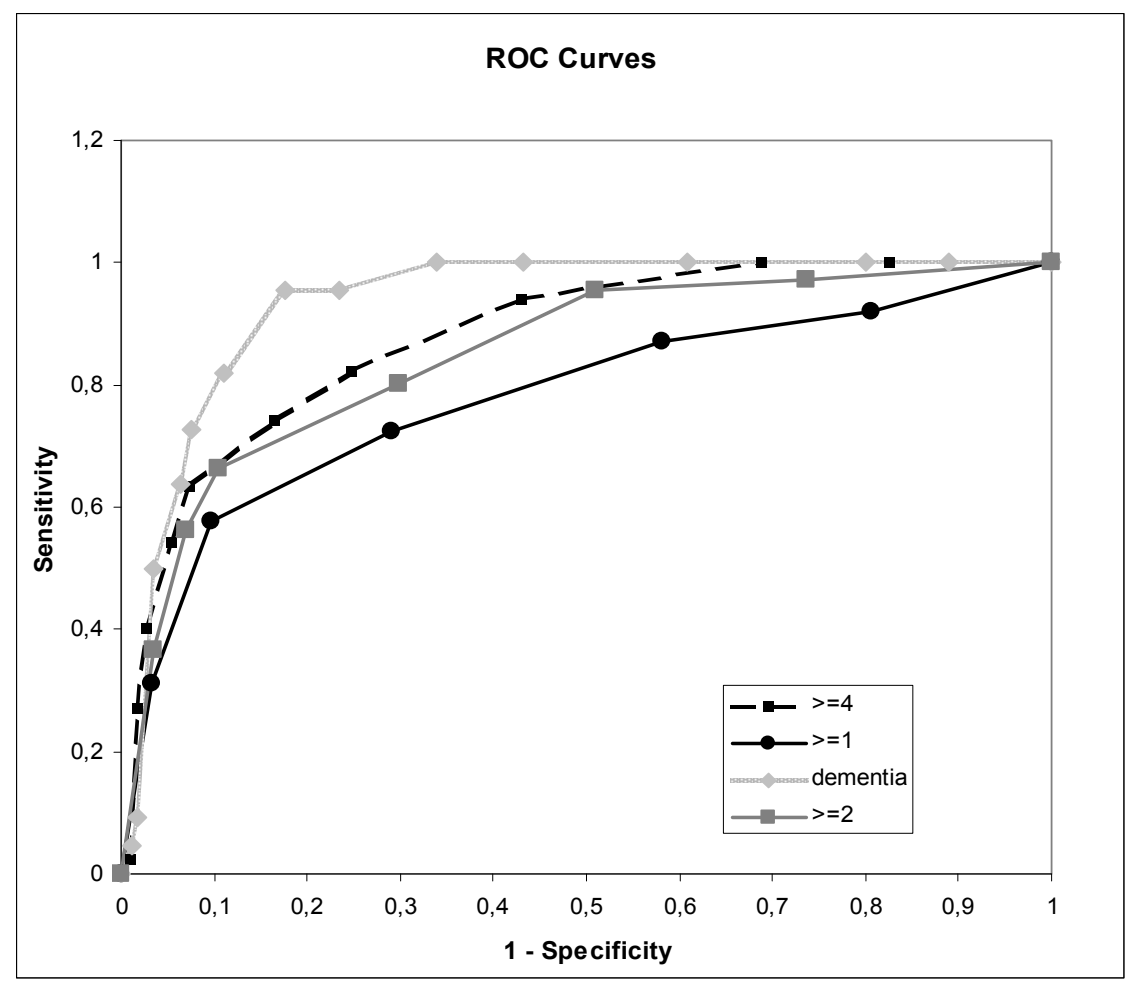

Figure 1. Receiver Operating curves of the MMSE in the screening for impaired cognitive domains and dementia

Table 5 shows optimum cut-off scores of the MMSE and their sensitivity and specificity in the screening for cognitive impairments and dementia.

In the screening for 1 impaired cognitive domain we found a moderate sensitivity of 0.72 at a cut-off score of $27 / 28$. At a cut-off of $28 / 29$ sensitivity increased to 0.87 but the specificity decreased to 0.42 . These values were comparable in patients with left-sided lesions and patients with right-sided lesions.

Table 5. Screening abilities of the MMSE at optimum cut-off points in the screening for impaired cognitive domains and dementia.

\begin{tabular}{llllll}
\hline Impaired domains & Cut-off & Sensitivity & Specificity & PPV & AUC \\
\hline$\geq 1$ & $27 / 28$ & 0.72 & 0.71 & 0.93 & 0.79 \\
$\geq 2$ & $27 / 28$ & 0.80 & 0.70 & 0.86 & 0.86 \\
$\geq 4$ & $26 / 27$ & 0.82 & 0.75 & 0.72 & 0.88 \\
Dementia & $23 / 24$ & 0.96 & 0.83 & 0.41 & 0.94 \\
\hline
\end{tabular}

$\mathrm{PPV}=$ positive predictive value, $\mathrm{AUC}=$ area under the curve 
To study whether baseline MMSE-scores could predict further deterioration or an improvement in cognitive functioning, correlations and regression analyses were performed using the MMSE as a continuous scale and after categorizing patients into baseline MMSE scores over 27, between 27 and 24, or lower than 24. There was no correlation between the baseline MMSE-score and an improvement or deterioration in cognitive functioning. Neither could baseline MMSE-scores predict an improvement or deterioration in cognitive functioning in a multivariate regression model adjusted for age, sex and education. A deterioration in MMSE score correlated with deterioration in cognitive functioning in time.

\section{Discussion}

The aim of this study was to investigate whether 1 month after stroke the MMSE could predict cognitive impairment and its course over 2 years. We found significant correlations between the MMSE score one month after the event and cognitive deficits at each follow-up visit 6, 12, and 24 months after. Furthermore, the MMSE predicted cognitive impairment and dementia in a multivariate regression model adjusted for age and education. These findings are comparable to a study by Tatemichi and co-workers, who found that a poor performance on the MMSE after one week after stroke was an important indicator for poor performance in cognitive functioning after three months [3].

After 1 year about $60 \%$ of patients with baseline MMSE scores less than 27 and $40 \%$ of those with MMSE scores of at least 27 showed an improvement in their cognitive performance. Patients with low MMSE scores more often deteriorated, whereas patients with higher scores more often were stable in the number of disturbed cognitive domains. However, baseline MMSE scores were not sensitive in the screening for a change in cognitive performance. Deterioration or improvement in the number of disturbed cognitive domains at later follow-up visits were not predicted by the MMSE 1 month after the event. A deterioration in MMSE score correlated with deterioration in cognitive functioning in time.

We found MMSE cut-off scores of 27 / 28 with a good sensitivity in the screening for at least 2 disturbed domains, and a cut-off of 26 / 27 in the screening for at least 4 disturbed domains. In the diagnosis for dementia we found a cut-off score of 23 / 24 with a sensitivity and specificity of 0.96 and 0.83 respectively. However, in the screening for 1 disturbed domain we found a cut-off score of 27 / 28 with a moderate sensitivity and specificity of 0.72 and 0.71 . The cut-off scores in our 
study are comparable to those in the literature $[50,198,199]$. Our results suggest that an MMSE score of less than 27 possibly indicates substantial cognitive disturbances and thorough screening for cognitive decline is warranted. The MMSE is moderately sensitive in the mildly cognitively affected patients.

The validity of the MMSE in the screening for cognitive decline and dementia in stroke is still debated in the literature $[52,193,198]$. It is said to be sensitive only when a patient is already severely impaired.[16] In the screening for dementia in general a cut-off of 23 / 24 is administered. However, the literature advises to adjust this cut-off to an individual patient's confounders[24] or to increase the cutoff value [199]. In our study, based on the sensitivity at the optimum cut-off points, the MMSE had moderate properties in screening for mild cognitive disturbances but was a sensitive screening tool in screening for moderate cognitive deterioration and dementia.

Nys and co-workers studied the validity of the MMSE in the first week after stroke in the screening for any disturbance in 1 of 6 cognitive domains in 34 stroke patients and 34 age and education matched controls [52].They could not find an optimum cut-off point in the screening for at least 1 out of 6 disturbed domains. Our study included a larger sample and contained a comparable amount of subcortical and cortical lesions. We did not examine the patients cognitively in the first days after stroke. The MMSE was performed in the same session as the neuropsychological test battery.

In another study comprising 112 stroke patients, the MMSE had a moderate sensitivity (0.62) and specificity (0.88) at a cut-off point of 24 in the screening for cognitive impairment [51].The authors concluded that the MMSE is not sensitive to memory complaints. Time between the MMSE (first week after stroke) and cognitive testing (within 3 months after stroke) varied substantially. Therefore, the relationship between the two may have been weakened, since spontaneous recovery may have occurred between tests.

Several studies report limitations of the MMSE in the screening for dementia and cognitive impairment especially in subcortical infarctions and small vessel disease where it would not differentiate between focal and diffuse lesions. Furthermore, it would be insensitive to right-sided lesions [40,193, 194]. We found no differences in screening properties between patients with left-sided lesions and with rightsided lesions.

Other studies report the MMSE to be sufficiently accurate as a screening instrument for cognitive impairment and dementia in stroke patients in the clinical situation, and support the ability of the MMSE to follow-up cognitive performance 
over time $[46,191,192,198,200]$. Other studies found the MMSE to be a useful screening tool when taking confounders into account such as age and education $[53,55,201,202]$, or when another additional screening test is performed [194, 203] and state that the difference in screening ability between left and right-sided lesions is exaggerated [55]. We found the MMSE to be an independent predictor for cognitive functioning.

The standard criteria and the ease of use make the test attractive [191, 204]. Furthermore, all short screening tests share deficits in sensitivity [205].

This study has some limitations to be mentioned. We excluded a substantial amount of patients with our exclusion criteria. Our study comprised of the relatively cognitively better part of stroke patients, since patients with a severe aphasia and an MMSE score < 15 were excluded. However, this generally applies to follow-up studies that require long-term cooperation. Patients are required to understand the tasks to measure their cognitive performance accurately. Including these patients rather would have increased the relationship between the MMSE and cognitive dysfunction and would have improved the sensitivity values in our study and the accuracy of the MMSE in the screening for cognitive impairment and dementia. It is not conceivable that the exclusion of the other patients influenced our results.

The 54 patients lost to follow-up were older, lower educated, and had more cognitive deficits and lower MMSE scores. This may have rather weakened our results.

In the cross-sectional analyses we found that most patients improved in cognitive functioning, and that patients with low baseline MMSE scores deteriorated more often than patients with high scores. These findings indicate that there are some differences in cognitive functioning over time between patients with high and low MMSE scores. However, nor in patients with high baseline MMSE scores nor in patients with low scores this is a linear effect predicted by baseline MMSE scores. Patients with high baseline MMSE scores more often were stable in their cognitive performance whereas in the patient group with low baseline MMSE scores less patients stabilized and cognitive performance either improved or deteriorated, showing a more dynamic course. Moreover, a change in cognitive functioning could not be predicted by the MMSE scores in a regression model adjusted for age and education. As is shown in this and other studies it is conceivable that a number of patients with cognitive impairment will deteriorate and a number of patients will improve [206, 207]. Most improvement is seen in the first months after stroke. The cross-sectional measurements after 24 months will partially 
overlap with those found at the 12 months' visit. Thus, although we only analyzed patients from whom we had test results at each follow-up visit we cannot be precise in determining the temporal effect on cognitive functioning in an individual patient and the relationship with the baseline MMSE from our study design. Alternatively, linear mixed models analyses could be attributed to analyse the course of cognitive functioning in time. However, this was problematic since dropouts differed substantially from patients who completed the follow-up in demographics and in MMSE scores. A study into the risk factors for decline was beyond our goals. Furthermore, our study was rather focussed on validating the screening ability of the MMSE. For this purpose the utilization of a fully completed data set was preferred. Although we assessed pre-stroke cognitive functioning in a retrospective manner by means of a structured interview based on the DSM-IV criteria for dementia with the patients' caregiver and not by means of a measurement scale, we cannot precisely distinguish how pre-stroke cognitive performance influences post-stroke cognitive performance, cognitive recovery, or how stroke influences pre-stroke cognition. To rule out such an effect, for instance, the change in MMSE scores between 6 and 1 month visits could be used to predict further cognitive change. However, this was beyond the goals of our study. Studying the effects between pre-stroke cognitive performance, stroke and post-stroke cognitive functioning would require a different study design.

We did not adjust for age and education in calculating the optimum cut-off. However, defining confounding factors for the MMSE was beyond the aim of this study. In the multivariable model, age and educational level did not predict cognitive functioning, whereas the baseline MMSE did.

Despite its limitations reported in the literature we found that the MMSE, which is easy to use, is a useful instrument to screen for moderate to severe cognitive deficits in stroke patients and to screen patients for more detailed neuropsychological assessment. A score on the MMSE of $<27$ one month after stroke is related to poor cognitive performance also in the longer term. However, it cannot be used to predict whether an individual patient will recover or deteriorate. 
The effect of the APOE- $\varepsilon 4$ allele and ACE I/D polymorphism on cognition during a two year follow-up in first-ever stroke patients

Adapted from:

A.M.J.J. Bour, S. M.C. Rasquin, L.Baars, M. P. J. van Boxtel, P.J. Visser, M. Limburg, F.R.J. Verhey. The effect of the APOE- 44 allele and ACE I/D polymorphism on cognition during a two year follow-up in first-ever stroke patients. Dementia and Geriatric Cognitive Disorders. In press. 


\section{Abstract}

Background: Cognitive impairment is commonly observed after stroke and has a negative impact on survival and rehabilitation. Some stroke patients deteriorate in cognitive functioning whereas others do not. Environmental and demographic risk factors cannot fully explain this. There is growing evidence that a genetic predisposition plays a role in the pathogenesis of post-stroke cognitive decline.

Objective: To study the influence of the APOE- $\varepsilon 4$ allele and the ACE-I/D polymorphism on cognitive functioning after stroke.

Methods: We included 194 first-ever stroke patients of whom information about APOE genotyping and ACE-I/D polymorphism was available in 92 and 129 patients, respectively. Patients were cognitively assessed at 1, 6, 12, and 24 months after the event. Linear mixed models with slope estimates were used to study the influence of the APOE- $\varepsilon 4$ allele and the ACE-I/D polymorphism on the MMSE score, CAMCOG, executive functioning, psychomotor speed, and verbal memory function during follow-up.

Results: Patients carrying the APOE- $\varepsilon 4$ allele more often suffered a lacunar infarction than non-carriers. The APOE- $\varepsilon 4$ allele had no effect on cognitive functioning during the follow-up. ACE-DD homozygosity was associated with a worse performance in executive functioning compared to patients with neither an APOE $\varepsilon 4$ allele nor the ACE DD genotype. There was no interaction between the APOE- $\varepsilon 4$ allele and the ACE-DD phenotype in the prediction of cognitive decline.

Conclusion: The ACE-DD genotype may be associated with post-stroke cognitive decline while the APOE-e4 allele is not. Further research is needed to examine the role of genetic risk factors for post-stroke cognitive decline and to determine why some patients deteriorate cognitively after stroke but others do not. 


\section{Introduction}

Cognitive decline is a commonly observed consequence of stroke. It is associated with a high morbidity, loss of independence, and mortality. Although most patients show a recovery in the first weeks after stroke, 25 percent of patients deteriorate, or even become demented $[41,187]$. Cognitive impairment caused by cerebrovascular disease is the second leading cause of dementia in older persons, after Alzheimer's disease (AD) $[208,209]$. Since stroke survival nowadays has improved, prevalence rates of vascular cognitive impairment $(\mathrm{VCI})$ and vascular dementia (VaD) will increase in the future [41]. Therefore, it is important to identify patients who are at risk of further cognitive deterioration.

It has not been fully elucidated whether patients suffering from post-stroke cognitive decline have other or perhaps additional risk factors compared to stroke patients who do not decline in cognitive functioning. Whereas $\mathrm{VaD}$ is caused by cerebral vascular lesions and leukoaraiosis, the pathology of $A D$ is characterized by the deposition of amyloid and neurofibrillary tangles in the brain. However, especially older $A D$ patients more often show atherosclerotic changes than nondemented contemporaries. Both $A D$ and $\mathrm{VaD}$ share vascular risk factors such as hypertension and high plasma cholesterol levels [56, 210-214]. Some studies report evidence for genetic influences, additional to conventional demographic risk factors, such as age and lower level of education on cognitive decline in both these patient groups [215-217]. The presence of the apolipoprotein E (APOE)- $\varepsilon 4$ allele is known to be a risk factor for $\operatorname{AD}$ pathology[218, 219]. APOE-ع4 is also associated with atherosclerotic changes and cardiovascular disease and brain infarctions [60]. Although an association between the presence of APOE- $\varepsilon 4$ and $\mathrm{VaD}$ has been reported, evidence for a contribution in the pathophysiological mechanism of $\mathrm{VaD}$ still is equivocal $[215,220]$.

The renin-angiotensin system (RAS) is reported to play a role in the pathogenesis of dementia through multiple effects related to vascular and metabolic factors and amyloid metabolism [221]. One of the participating enzymes in the RAS system is the angiotensin-converting enzyme (ACE). The ACE Insertion / Deletion (ACE I/D) gene polymorphism is thought to contribute to vascular disease via its influence on hypertension, endothelial function and smooth muscle proliferation and tone. Patients carrying the ACE-DD genotype have higher rates of ACE in their plasma and more often suffer from myocardial infarction, hypertension, diabetes mellitus, leukoaraiosis and stroke $[222,223]$. Furthermore, the ACE-D allele has been associated with cognitive decline in older persons and Richard et al. report an 
increased negative effect of an ACE DD genotype in combination with the presence of APOE $\varepsilon 4$ on cognitive decline $[63,224]$. Purandare et al. report a higher load of white matter hyperintensities in the presence of an ACE DD homozygosity [225]. Reports on the importance of the ACE-D allele and the ACE-DD genotype in AD and $\mathrm{VaD}$, however, are conflicting and several studies have shown that the effect of the ACE I/D polymorphism on VaD is neutral [217, 226-228].

In this study we investigated whether the APOE $\varepsilon 4$ and the ACE-DD genotype have an influence on cognitive decline after stroke. Only few studies examined the genetic influence on cognition in stroke patients. Arpa et al. found no difference in the prevalence of APOE $\varepsilon 4$ or the ACE D allele between stroke patients with and without dementia. Baum et al. also reported a neutral effect of APOE $\varepsilon 4$ on cognition after stroke, whereas Slooter et al found that the APOE $\varepsilon 4$ allele was a risk factor for dementia after stroke $[227,229,230]$. We examined the influence of the APOE- $\varepsilon 4$ allele and the ACE-DD genotype on the Mini-Mental State Examination (MMSE) score, the Cambridge Examination for Mental Disorders in the elderly (CAMCOG) and on the cognitive domains mental speed, executive functioning, and verbal memory function at 1, 6, 12, and 24 months after stroke. Multiple cognitive domains were tested since cognitive impairment due to vascular lesions can be clinically heterogeneous [231, 232]. We hypothesized that carrying one or both of these genotypes would aggravate the negative effect of stroke on cognition causing these patients to deteriorate more than non-carriers.

\section{Methods}

\section{Patients}

Between January 2000 and August 2001194 patients admitted to the Neurology Department of the Maastricht University Medical Centre with a supratentorial stroke were enrolled in this study. Participants in this study were participants in a prospective cohort study into cognitive deficits after stroke CODAS. Study and patient characteristics have been described earlier [42]. Patients were asked to participate in the study within 48 hours after the stroke had occurred.

All participants underwent a structured medical and neurological assessment including medical history, physical examination, laboratory studies including genotyping for APOE and ACE-I/D polymorphisms, and a brain CT scan. Brain CT was performed within 24 hours. APOE and ACE-I/D genotyping were determined 
on genomic DNA extracted from EDTA-anticoagulated blood using the polymerase chain reaction (PCR) technique. As an evaluating side-arm study of CODAS we started the genotyping in a consecutive way from the second year of follow-up onward. Since approximately half the patients had already completed the followup we have information about APOE and ACE I/D of 92 and 129 patients respectively. Although genotyping was performed in a consecutive way patients of whom we did not have information about APOE and ACE I/D were older (70.1y (SD 12.1) vs. 66.6y (SD 12.8), $p<0.001$ and 70.0y (SD 12.6)vs. 67.7y (SD 12.4), $p=0.01$ ). They did not differ in level of education, sex, and mean MMSE and CAMCOG scores.

Stroke was diagnosed by a neurologist, based on clinical evaluation and the brain CT scan. Inclusion criteria were a first hemispheral stroke, age over 40 years, adequate post-stroke fluency in Dutch, and an initial post-stroke MMSE score > 15 . Exclusion criteria were severe aphatic disorder, pre-stroke dementia, or other major neurological or psychiatric disorders, that could interfere with the neuropsychological testing.

Patients were followed-up 1, 6, 12, and 24 months after the event for a clinical and neuropsychological evaluation. Informed consent was obtained and was approved by the local medical ethics committee.

\section{Assessment of cognitive performance}

Within one month all participants were assessed with the MMSE [50] and a neuropsychological test battery, administered by a well trained neuropsychologist (SR). In this study, in addition to total scores on the MMSE and the CAMCOG, measures for mental speed, executive functioning, and memory were also included.

Mental speed was evaluated using the Stroop I of the Stroop Colour Word Test (SCWT) [233]. The Stroop test probes selective attention and interference susceptibility. The test involves three test sheets displaying 100 stimuli each: Stroop part I contains colour names (testing reading speed), Stroop part II contains coloured patches (testing colour naming speed) and Stroop part III contains colour names printed in incongruously coloured ink. For the Stroop part III the colour of the ink has to be named instead of the printed colour name. The variable of interest is the time needed to complete each subtest. To measure executive functioning the mean interference scores of the SCWT were used [172]. This score was defined by the formula: Stroop part III - (Stroop part I + Stroop part II) / 2. 
Verbal memory was tested with the Auditory Verbal Learning Test (AVLT) [234, 235]. This test was used to measure episodic memory. After presenting 15 monosyllabic words verbally the patient is asked to recall as many words as possible. This procedure was repeated five times. The variable of interest was the number of spontaneously recalled words after 20 minutes (delayed recall). To minimise a possible learning effect we used parallel versions of the AVLT.

Overall scores of the Dutch version of the CAMCOG were used to test global cognitive performance. This test has been shown to be sensitive for cognitive disorders in stroke patients [170]. The CAMCOG is the cognitive part of the Cambridge Examination for Mental Disorders of the Elderly (CAMDEX). The CAMCOG can be subdivided into the subscales orientation, language expression and understanding, long and short term memory, attention, calculation, praxis, abstract thinking and abstract perception.

A diagnosis of dementia was based on all available clinical data, including information about medical history, test performance, structured information from a patient's informant about daily life functioning, and clinical observation. Two experienced clinicians who were blinded to the MMSE test outcome independently made the diagnosis according to the DSM-IV criteria for dementia [73]. A diagnosis of VaD was based on the NINDS-AIREN criteria [84]. Agreement between the two raters was determined by kappa statistics. In this study agreement between the raters was excellent (kappa=0.88; $p=0.01$ ) [236]. If there was a discrepancy between raters a consensus meeting was arranged. If no consensus could be reached, a patient was considered as being not demented.

\section{Statistical Analyses}

Demographic variables were analyzed using descriptive statistics. For the comparison of variables between APOE $\varepsilon 4$ carriers and non-carriers, t-tests were used for continuous variables, and in case of dichotomous variables Chi-square tests were used. For the comparison between ACE I/D genotypes, multiple comparisons ANOVA were used in case of continuous variables and Chi-square tests were used for dichotomous variables.

Longitudinal effects of the APOE $\varepsilon 4$ allele and the ACE-DD genotype on cognitive performance were tested using slope analysis with Linear Mixed Models [237]. The analyses included the baseline and follow-up scores. The final model included fixed terms for intercept (baseline performance for an individual with a value of zero on all covariates), age, age (to control for quadratic age trends in the longitudinal 
data), time (in months since baseline), sex ( $0=$ female, $1=$ male), education (low and intermediate or high), the presence of one or more APOE $\varepsilon 4$ allele, the ACE-DD genotype, or both. Patients with neither the APOE- $\varepsilon 4$ allele nor the ACE-DD genotype were the reference group to which all other groups (only APOE4 present, only ACE DD present, both APOE4 and ACE DD present) were compared. Interactions between time and the presence of APOE- $\varepsilon 4$, time and the presence of ACE-DD genotyping, and between time and the presence of both APOE- $\varepsilon 4$ and the ACE-DD genotype were examined. Post-hoc analyses were repeated including the presence / absence of APOE- $\varepsilon 4$ and the presence / absence of ACE-DD separately. Furthermore, analyses were repeated including the presence / absence of preexistent asymptomatic vascular lesions. Because serial correlation was likely to be present between the repeated measurements, an unstructured covariance structure for the responses was chosen. P-values of 0.05 or smaller were considered statistically significant.

\section{Results}

Table 1 presents the patients characteristics of the study cohort.

Table 1. Patients characteristics of the study sample.

\begin{tabular}{ll}
\hline $\mathrm{N}=194$ & \\
\hline Sex (female) & $44.8 \%$ \\
Age (mean + SD) & $68.3(12.5)$ \\
Education (low / intermediate / high) & $56.2 \% / 31.4 \% / 12.4 \%$ \\
Cortical lesion & $41.4 \%$ \\
MMSE (mean + SD) & $25.5(3.5)$ \\
Camcog (mean + SD) & $83.2(12.2)$ \\
Rankin (mean + SD) & $3.1(1.4)$ \\
Diabetes & $14.4 \%$ \\
Smoking & $40.1 \%$ \\
Hypercholesterolaemia & $16.9 \%$ \\
Alcohol & $37.3 \%$ \\
Transient ischemic attack & $18.0 \%$ \\
Ischemic heart disease & $26.5 \%$ \\
Peripheral vascular disease & $10.0 \%$ \\
Asymptomatic cerebral vascular lesions & $44.0 \%$ \\
Cerebral atrophy & $62.2 \%$ \\
Diagnosis of dementia & $11.4 \%$ \\
\hline
\end{tabular}

Note. Rankin: handicap scale ( 0 no complaints -5 bedridden) 


\section{Chapter 7}

Information about the APOE- $\varepsilon 4$ allele was available for 92 patients (Table 2). APOE- $\varepsilon 4$ homozygosity occurred in 1 patient and was not analyzed separately. APOE- $\varepsilon 4$ carriers more often suffered a lacunar infarction compared to noncarriers (70.5 percent and 45.2 percent respectively, $\chi^{2}=4.8, \mathrm{df}=1, \mathrm{p}=0.03$ ). There were no other differences in demographic characteristics at baseline between APOE- $\varepsilon 4$ carriers and non-carriers.

Table 2. Patients characteristics at baseline according to APOE- $\varepsilon 4$ presence or absence.

\begin{tabular}{lll}
\hline APOE- $\varepsilon$ 4 allele (N=92) & Present ( $=55)$ & Absent (n=37) \\
\hline Sex (female) & $43.6 \%$ & $43.2 \%$ \\
Age (mean + SD) & $66.2(13.6)$ & $67.2(11.5)$ \\
Education low / intermediate / high & $50.9 / 32.7 / 16.4 \%$ & $51.4 / 37.8 / 10.8 \%$ \\
Cortical lesion & $29.5 \%$ & $54.8 \%$ \\
MMSE (mean + SD) & $26.0(3.1)$ & $25.6(3.4)$ \\
CAMCOG (mean + SD) & $84.7(11.7)$ & $84.1(11.2)$ \\
Rankin (mean + SD) & $3.0(1.4)$ & $2.4(1.5)$ \\
Diabetes Mellitus & $5.7 \%$ & $16.7 \%$ \\
Smoking & $47.4 \%$ & $34.4 \%$ \\
Hypercholesterolemia & $13.9 \%$ & $13.8 \%$ \\
Alcohol & $35.3 \%$ & $30.0 \%$ \\
Transient ischemic attack & $11.3 \%$ & $19.4 \%$ \\
Ischemic heart disease & $21.2 \%$ & $32.4 \%$ \\
Peripheral vascular disease & $9.6 \%$ & $14.3 \%$ \\
Asymptomatic cerebral vascular lesions & $45.1 \%$ & $44.1 \%$ \\
Cerebral atrophy & $72.0 \%$ & $71.0 \%$ \\
Diagnosis of dementia & $9.1 \%$ & $13.5 \%$ \\
\hline
\end{tabular}

Note. For the comparison of continues variables independent samples t-tests were used. For the comparison of dichotomous variables Chi square tests were used.

Rankin: handicap scale ( 0 no complaints -5 bedridden)

${ }^{*} \mathrm{Chi}^{2}=4.8, \mathrm{df}=1, \mathrm{p}=0.028$

Information about the ACE-I/D polymorphism was available for 129 patients (Table $3)$. There were no differences in demographic characteristics at baseline between patients with the II, ID, or DD genotype.

In the final model, the longitudinal effects of both the APOE- $\varepsilon 4$ allele with the ACEDD genotype, the APOE- 44 allele, and the ACE-DD genotype were analyzed compared to the reference group (patients with neither an APOE- $\varepsilon 4$ allele nor the ACE-DD genotype), in addition to the effects of age, age ${ }^{2}$, educational level and sex as covariates (Table 4). Slope estimations indicate the change during follow-up and $\Delta$ refers to the difference at the end of follow-up compared to the reference group. 
Table 3. Patients characteristics at baseline according to ACE I/D polymorphism.

\begin{tabular}{llll}
\hline ACE genotype (N=129) & DD $(\mathrm{n}=44)$ & ID $(\mathrm{n}=52)$ & II $(\mathrm{n}=33)$ \\
\hline Sex (female) & $43.2 \%$ & $50.0 \%$ & $39.4 \%$ \\
Age (mean + SD) & $68.4(11.1)$ & $66.6(13.0)$ & $68.4(13.6)$ \\
Education low / intermediate / & $50.0 / 40.9 / 9.1 \%$ & $51.9 / 32.7 / 15.4 \%$ & $66.7 / 27.3 / 6.1 \%$ \\
high & & & \\
Cortical lesion & $40.0 \%$ & $38.1 \%$ & $35.7 \%$ \\
MMSE (mean + SD) & $25.0(4.1)$ & $25.8(3.1)$ & $26.0(3.1)$ \\
Camcog (mean + SD) & $82.5(12.9)$ & $84.4(12.2)$ & $83.9(11.3)$ \\
Rankin (mean + SD) & $2.9(1.6)$ & $3.2(1.3)$ & $2.7(1.4)$ \\
Diabetes Mellitus & $13.6 \%$ & $6.1 \%$ & $12.1 \%$ \\
Smoking & $34.3 \%$ & $45.0 \%$ & $37.5 \%$ \\
Hypercholesterolaemia & $10.3 \%$ & $10.5 \%$ & $31.8 \%$ \\
Alcohol & $35.5 \%$ & $25.0 \%$ & $41.7 \%$ \\
Transient ischemic attack & $9.3 \%$ & $18.4 \%$ & $21.2 \%$ \\
Ischemic heart disease & $23.3 \%$ & $26.0 \%$ & $25.0 \%$ \\
Peripheral vascular disease & $14.3 \%$ & $8.5 \%$ & $9.1 \%$ \\
Asymptomatic cerebral vascular & $46.5 \%$ & $44.0 \%$ & $44.8 \%$ \\
lesions & & & \\
Cerebral atrophy & $65.9 \%$ & $69.6 \%$ & $76.9 \%$ \\
Diagnosis of dementia & $14.0 \%$ & $11.0 \%$ & $9.1 \%$ \\
\hline
\end{tabular}

Note. For the comparison of continues variables ANOVA with multiple comparisons was used. For the comparison of dichotomous variables Chi square tests were used.

Rankin: handicap scale ( 0 no complaints -5 bedridden)

Patients with a higher education performed better, whereas higher age had a negative effect on cognitive performance. CAMCOG scores and executive functions improved significantly over time during the follow-up in the reference group. There was no significant effect of the APOE- $\varepsilon 4$ allele on cognitive performance. Patients with ACE-DD homozygosity showed significantly less improvement in executive functioning compared to the reference group. There was no interaction between the APOE- $\varepsilon 4$ allele and the ACE-DD genotype on cognitive performance.

Post-hoc analyses were performed to study the change in cognitive performance in the presence or absence of the APOE- $\varepsilon 4$ allele and the presence or absence of the ACE-DD genotype separately and to study a possible effect of pre-existent vascular damage. Stroke recurred in 2 patients during follow-up and these were not analyzed separately. There was no difference in cognitive performance over time between APOE- $\varepsilon 4$ carriers and non-carriers. Patients with the ACE-DD genotype improved less in executive function than patients with one of both other genotypes (i.e. II or ID), but in other cognitive domains performance was comparable. 


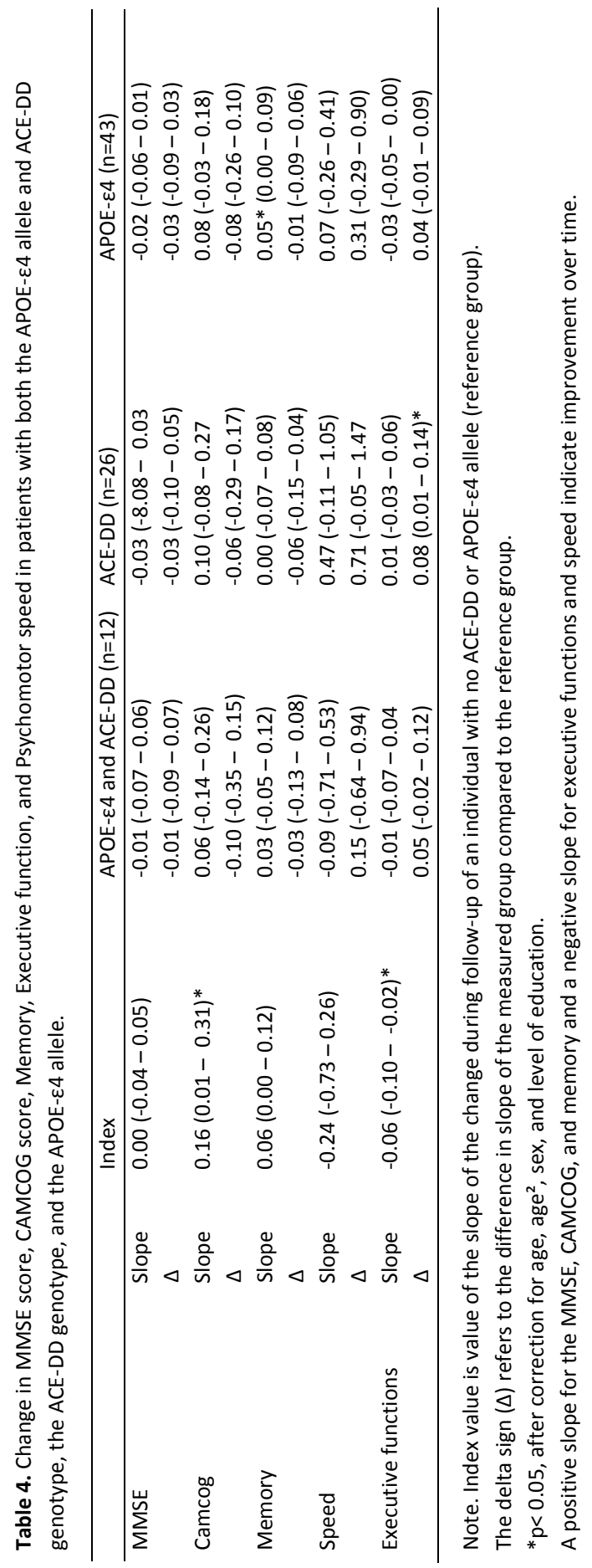


The presence of pre-existent asymptomatic lesions did not affect the longitudinal cognitive performances as measured by the assessment scales nor the separate domains.

\section{Discussion}

In this study we examined the effects of the APOE- $\varepsilon 4$ allele and the ACE-D allele on cognitive performance after stroke during a two year follow-up period. Patients carrying the APOE- $\varepsilon 4$ allele more often had a lacunar infarction. Presence of the APOE- $\varepsilon 4$ allele did not affect cognitive performance after stroke. There was a negative effect of the ACE-DD genotype on executive functioning.

This is the first study that focussed on cognitive functioning after stroke, using a relatively long follow-up period of two years. In the extant literature, most studies to date examined the effect of APOE- $\varepsilon 4$ status and the ACE I/D polymorphism on the pathogenesis of $A D$, mixed dementia or $\mathrm{VaD}$. Only few studies examined the effect of these genetic factors on cognitive decline and dementia in stroke patients finding conflicting results [227, 229, 230, 238-240]. Arpa et al. found no association between APOE or the ACE I/D polymorphism on post-stroke dementia in 150 stroke patients three months after the event [229]. The authors indicated that in case of stroke factors other than the APOE or ACE I/D polymorphism are of greater pathophysiological importance with respect to cognitive deterioration.

The pathogenesis of $A D$ is characterized by the deposition of amyloid protein in the brain and the formation of neurofibrillary tangles, whereas $V a D$ is characterized by vascular lesions and leukoaraiosis. However, nowadays there is growing evidence of shared environmental and genetic risk factors between AD and $\mathrm{VaD}$ like hypertension, diabetes mellitus, and atherosclerosis [56, 212, 241]. Moreover, histopathological studies report the involvement of vascular lesions in $A D$ and in $\mathrm{VaD}$ there is evidence for an increased accumulation of cerebral amyloid protein [61, 220, 242].

Whereas the relationship between APOE- $\varepsilon 4$ and $A D$ is extensively established, studies on the effect of $A P O E-\varepsilon 4$ on vascular dementia and the relationship to post-stroke cognition report ambiguous results [240, 243-245]. APOE is a lipoprotein which can be synthesized in the brain. APOE- $\varepsilon 4$ plays a role in the metabolism of the amyloid- $\beta$ protein, which accumulates in AD. It is negatively associated with various neurodegenerative brain diseases like dementia with Lewy bodies, tauopathies and Parkinson's disease, multiple sclerosis, and with brain 
injury. Furthermore, APOE-ع4 plays a role in lipid metabolism and cholesterol transport and is associated with atherosclerosis [60]. It is hypothesized that in the pathophysiology of $A D$ associated with disease onset late in life cognitive deterioration is caused by vascular pathology as a 'second hit' in addition to (a genetic predisposition for) increased amyloid deposition [64, 65]. In the pathogenesis of $\mathrm{VaD}$ and mixed dementia an interaction between a facilitating effect of APOE- $\varepsilon 4$ is suggested through an interaction between APOE- $\varepsilon 4$ and AD type pathology and vascular risk factors such as hypertension, atherosclerosis and diabetes [61, 220, 246, 247, 272]. In stroke APOE- $\varepsilon 4$ carriers are reported to be more vulnerable to the damaging effect of ischaemia causing larger infarct sizes [248]. Ballard et al. studied the effect of APOE- $\varepsilon 4$ on cognition in older stroke patients and found that APOE- $\varepsilon 4$ carriers performed cognitively worse and showed more cognitive deterioration after stroke compared to non-carriers [273]. Another study among 152 stroke patients found the presence of one or two APOE- $\varepsilon 4$ alleles to increase the risk of post-stroke cognitive decline. They found no association between APOE- $\varepsilon 4$ and pre-stroke cognition [274].

Several studies report a higher prevalence of the APOE- $\varepsilon 4$ allele in VaD patients, patients with mixed $A D$ and $\mathrm{VaD}$, and in post-stroke dementia [216, 230, 249, 250]. Other studies found no evident role of APOE- $\varepsilon 4$ in the pathophysiology of VaD or dementia after stroke [238-240, 244, 275-277], which is comparable to our results. Although neither the 'subcortical' domains mental speed and executive functioning were influenced by the APOE- $\varepsilon 4$ allele, it is possible that a potential relationship between cognitive performance and the APOE- $\varepsilon 4$ allele is masked in our cohort since APOE- $\varepsilon 4$ carriers less often had a cortical lesion, which is assumed to be stronger related to cognitive performance.

It has been suggested that in the pathogenesis of white matter lesions the effect of APOE4 may become important in the presence of the ACE-DD genotype [225, 251]. Richard et al. reported a synergistic negative effect of the ACE-DD genotype in combination with the APOE- $\varepsilon 4$ allele on cognitive decline in a large communitydwelling elderly cohort, whereas Gustafsen et al. reported a relationship between the ACE-II genotype, the presence of a APOE- $\varepsilon 4$ allele, and age having a negative effect on cognition [63, 252].

The ACE-I/D polymorphism is suggested to be related to the pathogenesis of memory impairment, cognitive decline and dementia, albeit that results are conflicting $[63,217,224,228,253]$. Whereas some studies report the association of the ACE-II genotype to be associated with dementia, particularly in VaD, the 
ACE-DD genotype is reported to be of pathophysiological influence on cognitive decline [217, 222, 224, 252, 254].

The renin-angiotensin system plays a major role in blood pressure homeostasis. ACE converts angiotensin I into angiotensin II, which is a vasoconstrictor. Moreover, ACE has an influence on endothelial function and smooth muscle proliferation in blood vessels. Patients exhibiting the DD genotype have higher levels of ACE in their plasma and more often suffer from cardiovascular disease. In the brain, the ACE-DD genotype is related to small-vessel pathology, leukoaraiosis, lacunar infarctions, and white matter lesions [222, 223]. This may explain why we found a slightly negative effect of the ACE-DD genotype on executive functioning in our study, since this is a feature of cognitive impairment in subcortical vascular pathology $[47,255]$.

A strong point of our study is that we examined the longitudinal effect of the APOE- $\varepsilon 4$ allele and ACE-DD genotype on cognitive performance with time, whereas other studies have a cross-sectional design focussing on the diagnosis of dementia or scores on a general cognitive screening tool. The diagnosis dementia may be too rough an endpoint thus missing patients with less severe cognitive impairment who do, however, experience problems in their daily routine. We used a very thorough cognitive assessment and explored the effect of the genetic factors on the cognitive domains memory, speed, and executive function separately, as we know that particularly in relation to stroke mild cognitive impairment is clinically heterogeneous $[41,255,256]$. Moreover, cognitive decline is not a stable entity but more of a dynamic spectrum in which dementia is the most extreme endpoint and not every patient suffering from cognitive decline will eventually become demented [208, 257].

One of the shortcomings of our study is that we did not collect genetic information concerning the APOE and ACE-I/D genotyping of the entire cohort. We started to collect genetic information at a later stage of the study as an evaluating sub-study. This results in a relatively small sample size of patients. Although genetic information was collected in a consecutive way, the patients of whom we lack this information were older compared to the patients of whom we did dispose of genetic information. Baseline and end-point cognitive performance as measured by the MMSE and CAMCOG did not differ. It is not conceivable that the distribution of the genetic variables of interest differed from the patients in the study, which makes bias of the contribution of a genetic predisposition to cognitive deterioration not likely. Our results may however be biased towards a better functioning group of patients, due to our exclusion criteria which excluded 
severely cognitively affected patients. A certain level of cognitive functioning is needed to complete a long follow-up period. These issues may have led rather to an underestimation of our results. The effect of progressive brain damage on cognition was reported earlier by our group was therefore not examined here. Patients with progressive brain damage did not deteriorate more in cognitive performance indicating more complex relations between ongoing vascular damage and cognitive deterioration [78]. Moreover, pre-existent vascular lesions did not influence further decline and stroke recurrence rates were low.

This is the first study examining the longitudinal effects of the APOE4 gene and the ACE-I/D polymorphism on cognitive functioning in stroke patients. We found a negative effect of the ACE-DD genotype on executive functioning, which is characteristic for cognitive decline post-stroke [258]. Our sample however was relatively small. Nevertheless, this may show new pathways towards a better understanding of the pathophysiology of post-stroke cognitive decline and treatment options. The effect of a genetic predisposition to cognitive decline in case of vascular brain damage should be evaluated more extensively. Higher age had a negative influence on cognitive performance whereas a higher educational level was protective. These and other results from the literature indicate that in case of stroke factors other than age, education, and lesion site are of greater importance in the pathophysiological mechanism for ongoing post-stroke cognitive decline. More longitudinal research in larger, well-defined follow-up samples is needed to evaluate the pathogenesis of post-stroke cognitive dysfunction in order to determine why some patients deteriorate but others do not. 


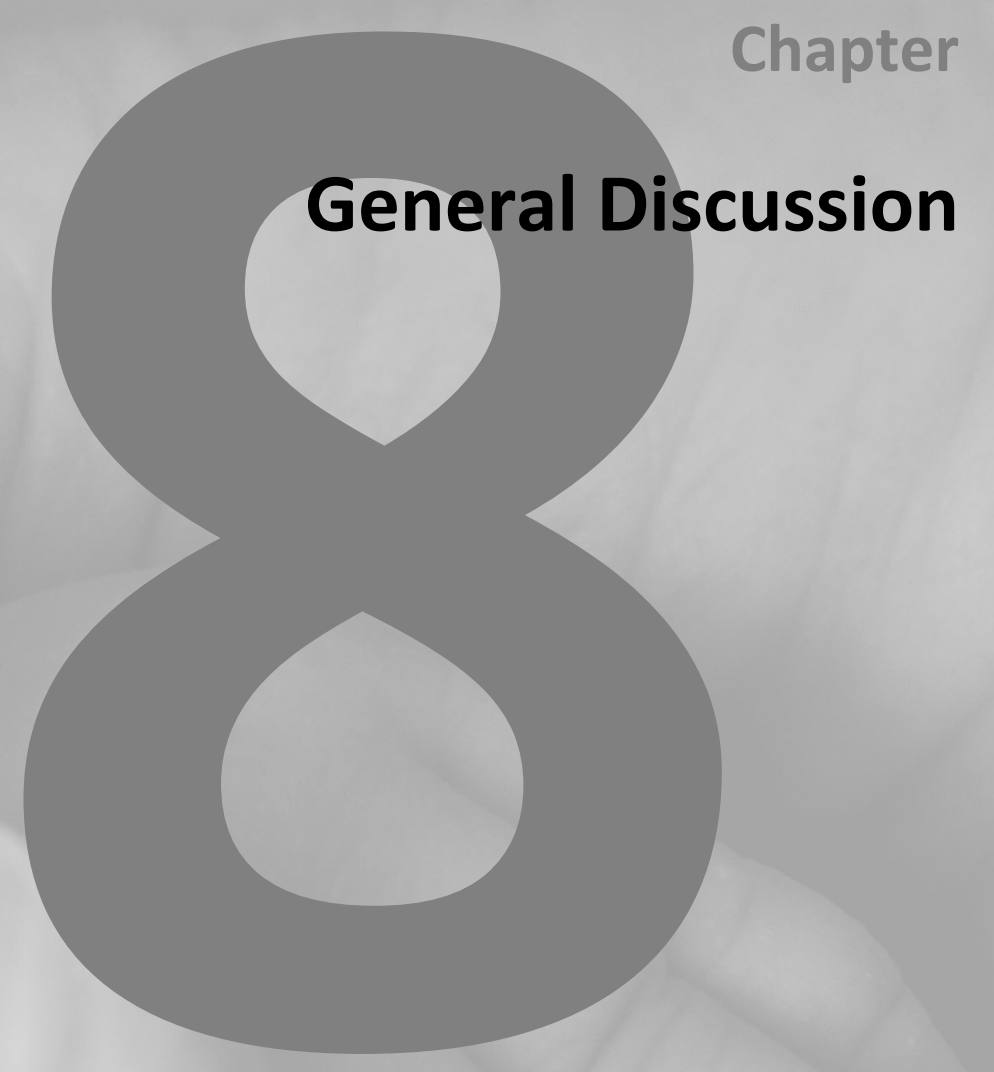


Chapter 8 
There is a great deal of literature on research into depression and cognitive decline after stroke which focuses on aetiology, pathogenesis, and demographic characteristics. Psychiatric and cognitive consequences of stroke are generally considered as two distinct clinical entities. Thus, there is quite a gap between the literature on post stroke depression and that on post stroke cognitive impairment. Both on the conceptual level and in clinical practice however, signs and symptoms of depression and cognitive deterioration overlap substantially. The aim of this thesis was to study symptoms of depression and cognitive decline, their course, how they interact and can be diagnosed clinically. In this chapter the main findings of our study and their clinical and research implications are discussed.

\section{Methodological considerations}

Before the findings and implications of this study can be discussed there are some limitations to be mentioned. Although ever more attention is being raised on neuropsychiatric sequelae after stroke most studies tend to focus either on depression or on cognitive deficits after stroke. There are only few studies that integrate these two entities [22, 102]. For this thesis, patients' data from two separate cohorts that were composed previously were available [76, 103]. One cohort studied depression after stroke and did not yield information about cognitive performance during follow-up. Several diagnostic tools were attributed including an experts clinical opinion. The other cohort focused on cognitive decline after stroke. Patients were administered a comprehensive neuropsychological test battery at four follow-up moments during two years and psychiatric symptoms were screened for by administering the SCL-90 self-rating scale to the patients at every follow-up, the validity of which has been demonstrated. Although both cohorts did not focus on the overlap and course of neuropsychiatric symptoms, both cohorts included assessments on both affective and cognitive changes, thus allowing for a valid post-hoc exploration.

Both cohorts used strict exclusion criteria thereby excluding patients too severely disabled in our opinion to be capable of participating in all assessments. Patients with severe generalized cognitive deficits and aphasia were excluded so that both cohorts consisted of mildly severely affected stroke patients hampering generalization of our findings to all stroke patients. Since depression is associated with level of handicap it is conceivable that we ruled out depressive patients. To date better screening instruments have been developed to better evaluate 
depressive complaints in aphasic patients [16]. Patients that were lost to follow-up in the $\mathrm{VCl}$ cohort were older, lower educated and had lower scores on the MMSE at baseline. However, inclusion of these possibly more severely affected patients would perhaps have resulted in more robust conclusions. Severely affected patients will be recognized in practice. However, these so-called mildly affected patients to whom this study is applicable, may be the ones in whom neuropsychiatric symptoms seem 'invisible' initially but who may experience difficulties later when trying to take up and continue their normal daily living. In this thesis we rather focused on depressive complaints, instead of the diagnosis of depression by the DSM-IV. In the chapters where the DSM-IV criteria for major and minor depression were utilized (Chapters 2, 3, and 4) the diagnosis was also mainly based on a patient's experience. Furthermore, we did not really differentiate major from minor depression. The DSM-IV utilizes this separation to give an indication of the severity of the depression. According to the DSM-IV major depressive episode is defined as having at least one of two core symptoms (i.e. depressed mood and loss of interest) in combination with at least four other symptoms of depression (sleep difficulties, loss of appetite / weight, fatigue, concentration difficulties, psychomotor retardation / agitation, low self-esteem / guilt, and thoughts of suicide) that last at least two weeks. Minor depression is defined as having at least one core symptom and at least two (but less than five) symptoms of depression [73]. As depression constitutes a dimensional phenomenon rather than a categorical disorder, such an approach may be too narrow. Complaints may seem subtle nevertheless causing great distress to a given subject. Also minor depression can cause loss of functionality in daily life and take a chronic course. Clinical practice and research settings however are confronted with the situation that subjective complaints preferably need to be quantified in order to measure them and make them objective. So, a certain categorization becomes almost inevitable. It is conceivable that this impedes sensitivity. Therefore, in our opinion in the evaluation of depression complaints should be handled rather as a continuum. We focused on depressive complaints, not so much on a depressive disorder, since a patient who exhibits depressive complaints can be hindered substantially while diagnostic criteria for depression are not strictly met. 


\section{Phenomenology of depression after stroke}

In depression after stroke not only psychological complaints but also physical symptoms such as gastro-intestinal complaints and fatigue are commonly observed. However, these symptoms are also commonly observed in nondepressed stroke patients, and thus it is not easy to determine how these symptoms should be attributed. This makes diagnosis of depression in this population difficult. The conclusion that stroke increases the risk of depression and causes a certain symptomatology is not informative about the nature of stroke as a risk factor. The pathophysiological mechanism behind these signs and symptoms raises the question whether certain symptoms observed in depressed stroke patients are due to a brain damage induced depression, which would implicate that PSD is a disease in itself, or to the stroke itself. In the literature the phenomenology of PSD is mainly investigated by comparing signs and symptoms to non-depressed stroke patients or to patients suffering from a primary depression $[10,11,119,127]$. These studies stress the importance of physical and vegetative symptoms but study results vary. No distinct symptom profile is evident. These studies, however, do not relate the observed phenomena to stroke. Stroke patients are at a higher risk of becoming depressed because of their vascular risk profile and the chronic character of the disease. To better understand the role of stroke in these depressed patients this should be considered. We compared signs and symptoms of 190 stroke patients to another vascular and chronically diseased cohort consisting of $200 \mathrm{MI}$ patients (Chapter 3). We found that depressed stroke patients more often suffered from loss of interest, psychomotor retardation, and gastro-intestinal complaints. After additional analyses including the non-depressed stroke and $\mathrm{MI}$ patients, these differences appeared to be stroke related. Non-depressed stroke patients exhibited the same symptoms as the depressed stroke patients, albeit less pronounced. This implies that probably other factors, such as personality traits, play an additional role in the pathophysiology of post-stroke depression and perhaps determine why some stroke patients are more at risk for depression after stroke than others (Chapter 2).

Another difficulty in the diagnosis of PSD is the phenomenological overlap with cognitive deficits that are commonly observed in stroke patients. The interaction between these entities is complex and bidirectional relationships have been reported [259-262]. Cognitive deterioration after stroke often is characterized by an impairment in executive functions, which in return is also a core feature in 
vascular depression [157, 177, 232, 263]. Outcome is worse in the presence of executive dysfunction [264-266]. As stated before, in comparison to MI patients we also found more signs of apathy in the stroke patients. In the depressed stroke patients however, these symptoms were accompanied by feelings of lowered mood (Chapter 3).

In the literature depression and cognitive deterioration after stroke are commonly studied separately. We found that depressive symptoms and executive dysfunctions co-occurred in $22 \%$ of 116 stroke patients (Chapter 5). A correct attribution of these symptoms either to depression or to a degenerative cause is important since these differ in treatment strategy and outcome. Patients with both depression and executive dysfunctions had a worse outcome implying that in these patients more robust treatment is warranted.

\section{The course of depression after stroke}

PSD treatment results are debatable [100]. Not all depressed stroke patients benefit from antidepressants. On the other hand, not every stroke patient will need medication. To distinguish patients who need more aggressive treatment insight into the course of PSD is essential. Literature overall reports a chronic disabling condition $[29,30]$. Furthermore, 'early' depressed patients in whom depression is diagnosed in the first months after stroke are distinguished from 'late' depressed patients in pathophysiology and phenomenology [2, 118]. In early onset depression biological factors (i.e. the lesion itself) would dominate as a causal factor, whereas in late onset depression other factors would be of more importance. In a one-year follow-up study of depressed stroke patients we found that the incidence of PSD was highest one month after stroke: half of patients who became depressed during that year were diagnosed as such in the first month (Chapter 4). In one in three patients who were depressed early after stroke symptoms recovered spontaneously within weeks. If depression in these patients were to be due to the lesion per se spontaneous recovery would be less probable. In $44 \%$ symptoms recurred later during the follow-up year. A weakness of our study is the relatively short follow-up period to determine what would be the course of depression in patients who were diagnosed as depressed at a later stage. Furthermore, after the first evaluation after one month only patients who scored above the cut-off scores of our screening instruments were evaluated further according to the DSM-IV criteria of major or minor depression. Thus, our study 
included only the more severely affected patients. Therefore the actual incidence of PSD and especially of more chronic symptomatology may be higher.

Patients in whom symptoms recurred or had a more chronic course were older and more often suffered from major depression. As stated before, patients in whom executive dysfunctions co-occurred had a worse outcome (Chapter 5). These patients may benefit most from treatment whereas in other cases a more conservative approach is justifiable.

\section{How can patients at risk for cognitive deterioration be identified?}

Cognitive deficits are observed in more than half of stroke patients. Most patients will improve but one in four patients will deteriorate with time and become demented. In clinical practice detection of cognitive deficits is important since these are associated with poor outcome. However, extensive neuropsychological testing in every stroke patient is not desirable nor feasible although Nys et al. report differently. Short screening instruments such as the MMSE are generally not considered to be sensitive enough in the diagnosis of mild cognitive impairment and dementia after stroke $[39,52]$. Cognitive deficits after stroke can be heterogeneous dependent on the location of the brain damage and the MMSE is generally considered to be too language dependent and not sensitive enough for deficits located in the right hemisphere. However, there is a broad experience concerning the MMSE since it is the most widely used cognitive test in clinical practice and research. Moreover, the MMSE was invented as a screening tool, not a diagnostic tool $[50,267]$. We evaluated the sensitivity of the MMSE in the detection for cognitive deterioration after stroke verified with an extended neuropsychological test battery (Chapter 6) and found an acceptable sensitivity and specificity in the screening for moderate cognitive impairment and dementia. A poor performance on the MMSE predicted poor performance later but could not predict whether a patient would improve or deteriorate. In the examination of cognitive functioning categorization of domains, which hampers diagnostic sensitivity and specificity, cannot completely be overcome. Cognitive functions overlap and ever so often patients are affected more globally in cognitive functioning, having some problems in several domains but not severe enough to speak of a 'disturbance'. This may not be detected due to categorization into domains. Attribution of more tests improves sensitivity of course, but extensive testing in these patients is not desirable since this demands a lot of effort from the 
often seriously ill patient. In short we concluded that the MMSE can be used as a screening tool to determine whether more extensive testing is required.

In the aetiology of dementia, whether caused by $\mathrm{AD}$ or by $\mathrm{VCl}$, ever more attention to genetic risk factors arises [268]. Perhaps a genetic vulnerability explains why some stroke patients deteriorate with time whilst others stabilize or recover. In AD it is hypothesized that cognitive decline is caused by vascular brain damage in addition to a genetic predisposition for increased brain amyloid deposition [64, 65]. Increasing evidence for the importance of vascular pathology in AD led to the exploration of the role of Apolipoptrotein $E$, which plays a key role in $A D$ pathophysiology, in $\mathrm{VCl}[61,269]$. Another genetic risk factor for $\mathrm{VCl}$ reported in the literature is the Angiotensin Converting Enzyme which plays a role in blood pressure regulation. In patients suffering from $\mathrm{VCl}$ the ACE-DD genotype is reported as a risk factor. However, study results are conflicting and clear conclusions cannot (yet) be drawn [63, 228, 252]. Most studies investigated the influence of genetic factors on global cognitive function measured by global screening instruments. Since we do not know the precise effects of these genes on cognition in the case of vascular brain pathology these tests may not be sensitive enough to detect a change. We explored the effects of the APOE- $\varepsilon 4$ allele and the ACE-DD phenotype on overall cognition and mental speed, executive functions, and memory specifically in 94 and 129 stroke patients respectively (Chapter 7). We found that the ACE-DD phenotype was associated with a lower level of improvement in executive functions. Since executive dysfunctions are typical for cognitive impairment due to vascular brain damage this may lead to future research directions. However, our study was explorative and to make clear conclusions about this matter would require a larger study population.

\section{Clinical implications}

Our studies bear several clinical implications. First, a clinician should be alert to cognitive and psychiatric comorbidity after stroke, not only in the acute phase but also in the long term. Since these sequelae hamper stroke recovery and outcome an early diagnosis is favourable in order to start a proper treatment. Extensive psychiatric or neuropsychological testing is not desirable since this is time and effort consuming, also for the patient. Regular screening instruments such as depression self-rating scales or the MMSE, however, can be applied as screening instruments to identify patients who do need extensive neuropsychiatric testing. 
It must be noted that diagnostic criteria for depression or cognitive impairment may be too narrow due to categorization, which is nevertheless inevitable in order to objectify these so often subjective complaints. For instance, although stroke patients in general commonly exhibit vegetative complaints and slowness these symptoms can underlie a depression or $\mathrm{VCl}$ even when the criteria for these entities are not strictly met. Subjective complaints about forgetfulness should be taken seriously and require further exploration. Apart from the utilization of scales a clinical evaluation is warranted. Since cognitive impairment and depression after stroke share similarities in their clinical presentation especially concerning executive functions (mental processing and speed), mood and neuropsychological function should both be tested in a patient in an integrated manner. Although symptoms overlap, deterioration in executive functions and apathy can be distinguished from depression clinically by evaluating mood.

If a patient is diagnosed depressed early after stroke a wait-and-see policy is justified. In the treatment of PSD there is no evidence for a standard prescription of antidepressants [100]. A patient should be informed about the prognosis and treatment considerations and symptoms must be monitored over time. Cognitive functions should also be considered and monitored during treatment. A clinician should be aware that especially in older patients or in more severely affected patients symptoms can recur or be chronic. Schepers et al. found that if depressive symptoms do not recover within 6 months after stroke, there is a high risk of chronic PSD [259]. Also in the co-occurrence of apathy outcome is worse. These patients may benefit from more aggressive therapeutic interventions. In the treatment of PSD it should be considered that the stroke itself is not the only causal factor and that multiple factors of causality which vary between subjects demand a multifactorial treatment approach.

\section{Research implications}

The ultimate goal of research into the neuropsychiatric consequences of stroke is prevention of these sequelae and effective treatment options in case they do occur. Diagnosis is a prerequisite for treatment. In the diagnosis of neuropsychiatric sequelae after stroke more insight into the discriminative value of symptoms is warranted. Cognitive functions and psychiatric disorders should be explored in an integrated way since they often co-occur and share similarities in symptomatology and causality. The interaction between these sequelae is 
complex. Both in $\mathrm{VCl}$ and in PSD, symptoms of executive dysfunction and apathy may result from vascular damage to fronto-subcortical circuits. However, in previous research into PSD by our group we could not support the vascular lesion theory or the vascular depression theory as a risk factor for PSD. Perhaps in the aetiology of $\mathrm{VCl}$ also more insight is needed into the effects of personality traits, coping mechanisms, and social networks to explain why some patients deteriorate in cognition after stroke while other patients do not.

In treatment strategies cognition and psychiatric symptoms also should be integrated more. Some small studies report improvement in cognition after treatment for depression. Perhaps cognitive deterioration plays a role in the marginal effects of antidepressants in PSD [185, 270]. Most studies concerning both cognition and depression test these entities globally. Better insight into the clinical and causal interaction requires extensive testing of both cognition and depression. As a result integrated (screening) measurement tools can be developed for clinical practice. Also better diagnostic criteria may be developed, since the current criteria for depression and cognitive decline are too narrow to embrace the full range of these heterogeneous entities.

In the exploration of risk factors and pathophysiological mechanisms of cognitive deterioration in vascular brain damage and stroke the ever increasing evidence for an overlap with $A D$ pathology indicates new pathways. One of these pathways may be genetic predispositions. We performed a small explorative study into the role of the $\varepsilon-4$ allele of the Apolipoprotein $E$ and the DD genotype of the Angiotensin Converting Enzyme I / D polymorphism on cognitive decline after stroke. We found a small negative effect of ACE-DD on executive functions, which is a characteristic feature of $\mathrm{VCl}$. ACE plays a role in blood pressure control. The role of ACE in cognition and the possibly positive effect of ACE-inhibitors should be examined more extensively [271]. This may be an opening into better understanding the aetiology of $\mathrm{VCl}$ and provide new treatment options. There are, to date, several small studies on genetic risk factors reporting varying results. These studies utilize global cognitive measurements. Larger studies are required which test cognition in detail, since effects on cognitive domains may be heterogeneous. Since some studies report that APOE may also play a role in the onset of (vascular) depression in the elderly studies into the genetic predisposition for neuropsychiatric consequences of stroke should, as stated before, integrate cognition and mood. 
Summary 
Cerebrovascular accidents (CVA) or stroke are the most important cause of death after myocardial infarctions and cancer in western countries. Approximately 41,000 people per year suffer a stroke in The Netherlands and approximately 190,000 are living with its consequences. Apart from substantial physical handicaps stroke often causes mental suffering, which may be permanent. Depression and cognitive problems are the most common neuropsychiatric ailments after stroke. Despite the increased attention given to these sequelae in the last few years and an increase in knowledge about them, emotional and cognitive problems appear to be more "invisible" in daily practice than one may think, and they often remain unnoticed especially in patients in whom physical impairments seem surprisingly minor.

Research into depression after stroke has mainly focused on pathophysiological mechanisms concerning especially the biological aspects; first in the form of the lesion location theory, which stated that primarily a lesion located left and frontally in the brain would be causal, and more recently the concept of vascular depression, which states that more diffuse damage to the brain would disrupt subcortical circuits resulting in mood disorders. Apart from these biological causes increasing attention has been paid to social factors and personality traits which make a patient more vulnerable to depression after stroke.

Less is known about the symptomatology of depression after stroke and its course. It has not been well established which patients would profit from what kind of treatment. Study results about the effects of anti-depressive drugs are inconclusive. Perhaps pharmacological intervention is not required for every depressive stroke patient since some of them will recover spontaneously. Therefore, better insight into the course of this type of depression is warranted.

Given that depression has a negative impact on stroke recovery early recognition is desirable. Depressed stroke patients often show emotional complaints in addition to physical complaints which are also observed in non-depressed stroke patients. It is not clear whether such symptoms are induced by the stroke itself or by post-stroke depression. Insight into the symptomatology is of clinical and of pathophysiological importance. For instance, some symptoms such as psychomotor slowing are not only observed in connection with depression but also in connection with cognitive decline after stroke. The depression-executive dysfunction syndrome is drawing increasing attention in stroke patients. Recognition and interpretation of this syndrome is important considering the prognosis and possible treatment. 
Cognitive decline is observed in more than half of stroke patients. One in four stroke patients even becomes demented. As the traditional determinants of vascular dementia $(\mathrm{VaD})$ could not adequately describe the problem, the much broader term "vascular cognitive impairment" or $\mathrm{VCl}$ was introduced as an allembracing term for any level and kind of cognitive deterioration caused by vascular disease. $\mathrm{VCl}$ can be clinically heterogeneous, but a syndrome characterized by so-called subcortical dysfunction, such as slowness and executive dysfunction, seems to be most prevalent. Less is known about the course of such a syndrome, in particular when someone does not fulfil the criteria of dementia and especially not if there is perhaps a concomitant mood disorder.

Despite the ever increasing consensus considering the concept of $\mathrm{VCl}$ and an ever improving definition of the clinical syndrome, in daily practice diagnosis remains problematic. A complete neuropsychological evaluation is time consuming and demands a lot of cooperation which is very burdensome in the acute disease stage. After the acute stage a neuropsychologist often is not available. The most widely applied tests such as the Mini Mental State Examination (MMSE) are considered not to be sensitive enough in $\mathrm{VCl}$. However, different study results vary considerably. Nevertheless, this test is utilized the most in daily practice and research.

$\mathrm{VaD}$ is, after Alzheimer's disease (AD), the most common cause of dementia. Research into the risk factors and possible pathophysiological mechanisms shows a lot of overlap between these two disease entities. For instance, in AD a clear relationship with cardiovascular risk factors and vascular damage in cerebro is found. Moreover, certain genetic factors such as Apolipoprotein E4 are known to increase the risk for AD. Furthermore, in patients with vascular cognitive deterioration potentially genetic risk factors are under investigation. This could provide more insight into the pathogenesis of these two dementia types, and could perhaps in the future offer an opportunity to identify patients who are more at risk for developing cognitive problems.

Given these perspectives and with these questions in mind this study continued on from previous findings from two separate cohort studies. One of these studies focused on post-stroke depression and the other focused on cognitive disturbances after stroke (CODAS).

In Chapter 2 we give an overview of the insights into post-stroke depression to date and previous findings of our team are discussed. Earlier investigations mainly focused on incidence and risk factors. Apart from biologic factors, such as a strategically located lesion or more diffuse vascular damage to the brain, social 
factors and personality traits also play an important role in the onset of poststroke depression. In research into post-stroke depression and in the care for stroke patients all aspects of this bio-psychosocial model should be given consideration.

In Chapter 3 we compared the signs and symptoms of depression in 190 stroke patients to the signs and symptoms of 200 myocardial infarction patients, as measured by the Hospital Anxiety and Depression Scale and the Hamilton Depression Rating Scale. We assumed that if post-stroke depression is a disease entity on its own that the symptoms would differ from the depressive symptoms seen after other chronic and vascular determined disease. Depressed stroke patients exhibited more loss of interests, psychomotor retardation, and gastrointestinal complaints. However, in a multivariable model including depressed patients as well as non-depressed patients no specific symptom profile for poststroke depression was found. Depressed stroke patients show other complaints than depressed myocardial infarction patients but this difference is due to differences between stroke and myocardial infarction patients in general.

The course of post-stroke depression was studied in Chapter 4. During one year 138 stroke patients were evaluated for depression at 1, 3, 6, 9, and 12 months after the event. During that year depression was diagnosed in $36.2 \%$ of patients. In half of these cases the diagnosis was made already 1 month after the event and in $30 \%$ of this group of early depressed patients the patients recovered within a few weeks. Strikingly, the diseased recurred in $44 \%$ within the follow-up year. Forty percent were chronically depressed. We concluded that it is true that depression is common after stroke and noticed it often recovers spontaneously within weeks. Particularly, in the more severe cases one should be mindful that the disease can recur and that the condition can continue for a long period. This group may benefit more from more intensive care and may need therapeutic interventions.

As stroke patients often exhibit executive dysfunction which can also result from depression the relationship between executive dysfunction and depression and the course of the so-called depression-executive dysfunction syndrome were examined in Chapter 5 . One hundred and sixteen patients were followed during 2 years and after $1,6,12$, and 24 months a complete neuropsychological test battery was administered. Depressive complaints were evaluated using the depression subscale of the SCL-90. Twenty two percent of these patients showed both depressive symptoms and executive dysfunction after 1 month, 24\% were depressed, and $11 \%$ showed executive dysfunction. Executive dysfunction was predicted by depression. Patients with both depression and executive dysfunction 
had a worse prognosis compared to patients with solely depression or executive dysfunction.

In Chapter 6 we examined the accuracy of the MMSE as a screening tool for cognitive deterioration in stroke patients. In general this test is said not to be of use to establish cognitive deterioration in this patient group. Results from previous investigations vary. However, it is the most widely utilized scale in daily practice and in research settings. In 194 stroke patients a complete neuropsychological test battery and an MMSE were performed 1 month after the stroke. These patients were again tested neuropsychologically completely after 6, 12, and 24 months. The MMSE score 1 month after the event predicted cognitive functioning later. However, improvement or deterioration was not predicted. We found a cut-off score of 27 / 28 with a sensitivity of 0.72 in the screening for 1 disturbed cognitive domain. In screening for 4 disturbed domains or dementia we found a cut-off score of 26 / 27 and 23 / 24 respectively with sensitivities of 0.82 and 0.96 . We concluded that the MMSE is useful as a screening tool for moderately severe cognitive decline and dementia after stroke and that a low score predicts a low score in the long term, but that it cannot predict improvement or deterioration.

Because of ever increasing evidence for an overlap in the pathophysiology between Alzheimer's disease and $\mathrm{VCl}$ we examined the effect of the APOE- 4 allele on cognitive functioning after stroke in time (Chapter 7). The effects on the MMSE, CAMCOG and the domains memory, speed, and executive function were tested. As several studies had found a relationship between $\mathrm{VCl}$ and the DD phenotype of the Angiotensin Converting Enzyme (ACE-DD) this effect on cognitive functioning was also explored. In addition, it was investigated whether there perhaps would be a synergistic effect on cognitive function in case a patient was an APOE- $\varepsilon 4$ carrier and also had the ACE-DD phenotype. We found that patients with an ACE-DD phenotype showed less improvement in executive function. The APOE- $\varepsilon 4$-allele had no influence on cognitive performance. There was no interaction between the APOE-ع4-allele and the ACE-DD phenotype. Future research should focus on whether or not there is perhaps a genetic explanation why some people do and others do not deteriorate cognitively after stroke.

In Chapter 8 the findings and shortcomings of this thesis and their implications for daily practice and future research are discussed. 



\section{Samenvatting}


Cerebrovasculaire accidenten (CVA) ofwel beroerten vormen op hartinfarcten en kanker na de belangrijkste doodsoorzaak in Westerse landen. Per jaar maken in Nederland ongeveer 41.000 mensen een beroerte door en ongeveer 190.000 mensen leven met de gevolgen ervan. Behalve aanzienlijke lichamelijke handicaps veroorzaakt een beroerte vaak ook mentaal leed, al dan niet blijvend. Depressie en cognitieve problemen zijn de meest voorkomende neuropschychiatrische aandoeningen na een beroerte. Ondanks dat er in de laatste jaren steeds meer aandacht is besteed aan deze sequelen en er meer over bekend is, blijken emotionele en cognitieve problemen in de dagelijkse praktijk toch "onzichtbaarder" te zijn dan we denken en blijven ze vaak onopgemerkt, vooral in patiënten bij wie de lichamelijke gebreken lijken "mee te vallen".

Onderzoek naar depressie na een beroerte heeft zich vooral gericht op pathofysiologische mechanismen en dan met name de biologische kant daarvan, aanvankelijk in de vorm van de laesie locatie theorie welke inhield dat met name een strategische laesie links frontaal in het brein oorzakelijk zou zijn en tegenwoordig in de vorm van het concept vasculaire depressie welke inhoudt dat meer diffuse schade in het brein subcorticale circuits zouden verstoren waardoor stemmingsstoornissen zouden ontstaan. Naast deze biologische oorzaken is er steeds meer aandacht gekomen voor sociale factoren en persoonlijkheidskenmerken welke een patiënt gevoeliger maken voor het krijgen van een depressie na een beroerte.

Minder is er bekend over de symptomatologie van depressie na een beroerte en het beloop ervan. Het is niet goed bekend welke patiënten baat bij welke vorm van behandeling. Studieresultaten naar het effect van antidepressiva bij deze patiëntengroep zijn niet conclusief. Wellicht is een medicamenteuze interventie niet in iedere depressieve CVA patiënt noodzakelijk omdat een deel van deze patiënten spontaan zal herstellen. Derhalve is meer inzicht in het beloop van deze vorm van depressie nodig.

Aangezien depressie het herstel na een beroerte negatief beïnvloedt is vroege herkenning gewenst. CVA patiënten met een depressie tonen naast emotionele klachten veelal lichamelijke klachten welke ook gezien worden bij niet-depressieve CVA patiënten. Het is niet duidelijk of dergelijke symptomen door het CVA op zich of door de post-CVA depressie worden geïnduceerd. Meer inzicht in de symptomatologie is niet alleen van klinisch belang maar ook van pathofysiologisch belang. Zo worden bepaalde symptomen zoals traagheid in doen en denken niet alleen in het kader van een depressie gezien maar ook in het kader van cognitieve achteruitgang na een beroerte. Het depressie-executieve disfunctie syndroom is 
een beeld dat steeds meer aandacht krijgt in CVA patiënten. Herkenning en interpretatie van dit beeld is belangrijk met het ook op de prognose en eventuele behandeling.

Cognitieve achteruitgang wordt gezien in meer dan de helft van alle CVA patiënten. Een vierde van alle CVA patiënten wordt zelfs dement. Omdat de klassieke determinanten voor vasculaire dementie $(\mathrm{VaD})$ niet het gehele probleem konden omvatten werd de veel ruimere term "vascular cognitive impairment " ofwel $\mathrm{VCl}$ geïntroduceerd als een allesomvattend begrip voor cognitieve achteruitgang in elke vorm en mate door vaatlijden bepaald. $\mathrm{VCl}$ kan zich klinisch op velerlei manieren uiten, maar een beeld dat bepaald wordt door zogenaamde subcorticale functiestoornissen zoals traagheid en executieve functiestoornissen lijkt het meest voor te komen. Over het beloop van een dergelijk beeld met name wanneer iemand dus niet voldoet aan de criteria van dementie is niet veel bekend. Met name niet als er mogelijk tevens sprake is van stemmingsproblematiek.

Ondanks dat er steeds meer consensus bereikt wordt over het concept $\mathrm{VCl}$ en het beeld steeds beter gedefinieerd wordt blijft de diagnostiek in de klinische praktijk een probleem. Een volledig neuropsychologisch onderzoek is tijdrovend en vergt veel coöperatie waardoor het in het acute stadium van de ziekte erg belastend is. $\mathrm{Na}$ het acute stadium is er vaak geen neuropsycholoog voorhanden. Van de meest gebruikte testen zoals bijvoorbeeld de Mini Mental State Examination (MMSE) wordt verondersteld dat ze niet gevoelig genoeg zijn voor $\mathrm{VCl}$. Resultaten uit verschillende studies wisselen echter sterk. Toch wordt deze test het meest gebruikt in de dagelijkse praktijk en in onderzoek.

$\mathrm{VaD}$ is op de ziekte van Alzheimer na de meest voorkomende oorzaak van dementie. Onderzoek naar de risicofactoren en mogelijke pathofysiologische mechanismen toont echter veel overlap tussen deze twee ziektebeelden. Zo wordt er bij de ziekte van Alzheimer een duidelijke relatie gevonden met risicofactoren voor hart- en vaatziekten en met vaatschade in cerebro. Verder is van bepaalde genetisch bepaalde factoren zoals het Apolipiproteïne E4 bekend dat ze het risico op de ziekte van Alzheimer vergroten. Ook bij patiënten met vasculair bepaalde cognitieve achteruitgang wordt gezocht naar eventuele genetisch bepaalde risicofactoren. Dit zou meer inzicht kunnen brengen in het pathofysiologisch mechanisme van deze beide dementieën, maar biedt in de toekomst wellicht ook de mogelijkheid mensen die meer risico lopen op cognitieve problemen te identificeren.

Met deze achtergrond en vragen bouwde deze studie verder op bevindingen die reeds eerder gedaan zijn uit twee verschillende cohortstudies. De ene studie 
richtte zich op post-CVA depressie en de andere op cognitieve stoornissen na een beroerte (CODAS).

In hoofdstuk 2 geven we een overzicht van de inzichten in post-CVA depressie op dit moment en worden de bevindingen uit eerder door ons team verricht onderzoek besproken. Eerder onderzoek heeft zich vooral gericht op incidentie en risicofactoren. Behalve biologische factoren zoals een strategische laesie dan wel meer diffuse vaatschade in het brein spelen ook sociale en persoonlijkheidskenmerken een invloedrijke rol in het ontstaan van post-CVA depressie. In onderzoek naar post-CVA depressie en in de begeleiding van CVA patiënten moet er meer rekening gehouden worden met alle aspecten van dit biopsychosociale model.

In hoofdstuk 3 vergeleken we de depressiesymptomen van 190 CVA-patiënten gemeten met de Hospital Anxiety and Depression Scale en de Hamilton Depression Rating Scale met de depressiesymptomen van 200 myocardinfarctpatiënten. We veronderstelden dat als post-CVA depressie een op zich zelf staand beeld zou zijn dat de symptomen zouden verschillen van de depressieve symptomen gezien na een eveneens chronische en vasculair bepaalde ziekte. Depressieve CVA-patiënten toonden meer interesseverlies, psychomotore retardatie en gastro-intestinale klachten. Echter, in een multivariabel model waarin naast depressieve patiënten ook niet-depressieve patiënten werden geanalyseerd werd geen specifiek symptomenprofiel gevonden voor post-CVA depressie. Depressieve CVA patiënten tonen wel andere symptomen dan depressieve myocardinfarctpatiënten maar dit verschil wordt veroorzaakt door verschillen tussen CVA-patiënten en myocardinfarctpatiënten algemeen.

Het beloop van post-CVA depressie werd onderzocht in hoofdstuk 4. Gedurende een jaar werden 138 CVA patiënten $1,3,6$, 9, en 12 maanden na de gebeurtenis onderzocht op een depressie. Gedurende dat jaar werd in $36.2 \%$ van de patiënten de diagnose depressie gesteld. In de helft van deze gevallen werd reeds na 1 maand de diagnose gesteld en in $30 \%$ van deze groep vroeg depressieve patiënten was de ziekte binnen enkele weken hersteld. Opvallend genoeg recidiveerde de aandoening binnen dat jaar in $44 \%$. Veertig procent was langdurig depressief. We concludeerden dat depressie na een CVA weliswaar vaak voorkomt, maar vaak vanzelf herstelt binnen enkele weken. Met name in de meer ernstige gevallen moet men erop bedacht zijn dat de aandoening terug kan komen en dat de aandoening lang kan voortduren. Deze groep zal meer baat hebben bij een intensievere begeleiding en therapeutische interventies behoeven. 
Omdat in CVA-patiënten vaak executieve functiestoornissen gezien worden die ook een gevolg van een eventuele depressie kunnen zijn werden in hoofdstuk 5 het verband tussen executieve functiestoornissen en depressie onderzocht en het beloop van het zogenaamde depressie-disexecutief syndroom. Honderd zestien mensen werden gedurende twee jaar gevolgd en na 1, 6, 12, en 24 maanden kregen zij een volledig neuropsychologisch onderzoek. Depressieve klachten werden geëvalueerd met de depressieschaal van de SCL-90. Tweeëntwintig procent van deze patiënten toonden na 1 maand zowel depressieve klachten als executief disfunctioneren, $24 \%$ was depressief en $11 \%$ toonde een executief disfunctioneren. Executief disfunctioneren werd voorspeld door depressie. Mensen met en depressieve klachten en executief disfunctioneren hadden een slechtere prognose dan mensen met alleen een depressie of alleen executief disfunctioneren.

In hoofdstuk 6 onderzochten we de gevoeligheid van de MMSE als screeningsinstrument voor cognitieve achteruitgang in CVA-patiënten. Over het algemeen wordt gezegd dat deze niet bruikbaar is om cognitieve achteruitgang vast te stellen in deze patiëntengroep. Resultaten uit eerder onderzoek zijn wisselend. Toch is het de meest gebruikte schaal in de klinische praktijk en in onderzoeksverband. Bij 194 CVA-patiënten werden 1 maand na het CVA een volledig neuropsychologisch onderzoek en een MMSE verricht. Deze mensen werden na 6, 12 en 24 maanden wederom volledig neuropsychologisch getest. De MMSE score zoals gemeten 1 maand na het CVA voorspelde het cognitieve functioneren later. Echter, vooruitgang of achteruitgang kon er niet mee worden voorspeld. We vonden een cut-off score van 27 / 28 met een sensitiviteit van 0.72 als gescreend werd voor 1 gestoord cognitief domein. Als gescreend werd voor 4 gestoorde domeinen of dementie vonden we een cut-off score van respectievelijk 26 / 27 en 23 / 24 met een sensitiviteit van 0.82 en 0.96 . We concludeerden dat de MMSE goed bruikbaar was als screeningsinstrument voor matig ernstige cognitieve achteruitgang en dementie na een CVA en dat een lage score voorspellend is voor een lage score op termijn, maar niet kan voorspellen of een patiënt zal verbeteren of verslechteren.

Gezien de toenemende aanwijzingen voor een overlap in de pathofysiologie tussen de ziekte van Alzheimer en $\mathrm{VCl}$ onderzochten we in hoofdstuk 7 wat het effect is van het APOE- $\varepsilon 4$-allel op het cognitieve functioneren na een CVA in de tijd. Getest werd het effect op de MMSE, de Camcog en de domeinen geheugen, snelheid en executieve functies. Aangezien er in eerdere studies een verband is gevonden tussen $\mathrm{VCl}$ en het DD fenotype van het Angiotensin Converting Enzyme (ACE-DD) 
werd ook het effect hiervan onderzocht op het cognitieve functioneren. Tevens werd er gekeken of er een synergistisch effect op het cognitieve functioneren zou bestaan indien een patiënt en drager is van het APOE- $\varepsilon 4$ allel en het ACE-DD fenotype heeft. We vonden dat het ACE-DD fenotype een negatieve invloed had op het executieve functioneren. Het APOE- $\varepsilon 4$ allel had geen invloed op het cognitieve functioneren. Er was geen interactie tussen het APOE- $\varepsilon 4$ allel en het ACE-DD fenotype. Toekomstig onderzoek zal zich er op moeten richten of er misschien een genetische verklaring is waarom sommige mensen wel en andere niet achteruitgaan in cognitie na een CVA.

In hoofdstuk 8 beschrijven we de bevindingen en tekortkomingen van dit proefschrift en hun betekenis voor de dagelijkse praktijk en toekomstig onderzoek. 
References 


\section{References}

1. Bots, M. and S. van Dis, Beroerte:cijfers en feiten. Nederlandse Hartstichting, 2006.

2. Robinson, R.G., Poststroke depression: prevalence, diagnosis, treatment, and disease progression. Biol Psychiatry, 2003. 54(3): p. 376-87.

3. Tatemichi, T.K., et al., Cognitive impairment after stroke: frequency, patterns, and relationship to functional abilities. J Neurol Neurosurg Psychiatry, 1994. 57(2): p. 202-7.

4. Rasquin, S.M., et al., Vascular cognitive disorders: memory, mental speed and cognitive flexibility after stroke. J Neurol Sci, 2002. 203-204: p. 115-9.

5. Aben, I., et al., A comparative study into the one year cumulative incidence of depression after stroke and myocardial infarction. J Neurol Neurosurg Psychiatry, 2003. 74(5): p. 581-5.

6. Pohjasvaara, T., et al., Frequency and clinical determinants of poststroke depression. Stroke, 1998. 29(11): p. 2311-2317.

7. O'Brien, J., B. Reisberg, and T. Erkinjuntti, Vascular burden of the brain. Int Psychogeriatr, 2003. 15 Suppl 1: p. 7-10.

8. Pohjasvaara, T., et al., Depression is an independent predictor of poor long-term functional outcome post-stroke. Eur J Neurol, 2001. 8(4): p. 315-9.

9. Aben, I., et al., Research into the specificity of depression after stroke: a review on an unresolved issue. Prog Neuropsychopharmacol Biol Psychiatry, 2001. 25(4): p. 671-89.

10. Spalletta, G., A. Ripa, and C. Caltagirone, Symptom profile of DSM-IV major and minor depressive disorders in first-ever stroke patients. Am J Geriatr Psychiatry, 2005. 13(2): p. 108-15.

11. Paradiso, S., T. Ohkubo, and R.G. Robinson, Vegetative and psychological symptoms associated with depressed mood over the first two years after stroke. Int J Psychiatry Med, 1997. 27(2): p. 137-57.

12. Fedoroff, J.P., et al., Are depressive symptoms nonspecific in patients with acute stroke? Am J Psychiatry, 1991. 148(9): p. 1172-6.

13. Robinson, R.G., The clinical neuropsychiatry of stroke. Cognitive, behavioral, and emotional disturbances following vascular brain injury. 2006.

14. Chemerinski, E. and S.R. Levine, Neuropsychiatric disorders following vascular brain injury. Mt Sinai J Med, 2006. 73(7): p. 1006-14.

15. Chemerinski, E. and R.G. Robinson, The neuropsychiatry of stroke. Psychosomatics, 2000. 41(1): p. 5-14.

16. Turner-Stokes, L., Poststroke depression: getting the full picture. Lancet, 2003. 361(9371): p. 17578.

17. Rao, R., Cerebrovascular disease and late life depression: an age old association revisited. Int J Geriatr Psychiatry, 2000. 15(5): p. 419-33.

18. Vataja, R., et al., Magnetic resonance imaging correlates of depression after ischemic stroke. Archives of General Psychiatry, 2001. 58(10): p. 925-931.

19. Alexopoulos, G.S., et al., 'Vascular depression' hypothesis. Arch Gen Psychiatry, 1997. 54(10): p. 915-22.

20. Krishnan, K.R., J.C. Hays, and D.G. Blazer, MRI-defined vascular depression. Am J Psychiatry, 1997. 154(4): p. 497-501.

21. Alexopoulos, G.S., et al., Clinically defined vascular depression. Am J Psychiatry, 1997. 154(4): p. 562-5.

22. Vataja, R., et al., Depression-executive dysfunction syndrome in stroke patients. Am J Geriatr Psychiatry, 2005. 13(2): p. 99-107.

23. Dieguez, S., et al., Is poststroke depression a vascular depression? J Neurol Sci, 2004. 226(1-2): p. 53-8.

24. Baldwin, R.C., Is vascular depression a distinct sub-type of depressive disorder? A review of causal evidence. Int J Geriatr Psychiatry, 2005. 20(1): p. 1-11. 
25. Aben, I. and F. Verhey, Depression after a cerebrovascular accident. The importance of the integration of neurobiological and psychosocial pathogenic models. Panminerva Med, 2006. 48(1): p. 49-57.

26. Whyte, E.M. and B.H. Mulsant, Post stroke depression: epidemiology, pathophysiology, and biological treatment. Biol Psychiatry, 2002. 52(3): p. 253-64.

27. Hackett, M., Frequency of depression after stroke. stroke, 2005. 36: p. 1330-1340.

28. Robinson, R.G., P.L. Bolduc, and T.R. Price, Two-year longitudinal study of poststroke mood disorders: diagnosis and outcome at one and two years. Stroke, 1987. 18(5): p. 837-43.

29. Astrom, M., R. Adolfsson, and K. Asplund, Major depression in stroke patients. A 3-year longitudinal study. Stroke, 1993. 24(7): p. 976-982.

30. Berg, A., et al., Poststroke depression: an 18-month follow-up. Stroke, 2003. 34(1): p. 138-43.

31. Hackett, M.L., C.S. Anderson, and A.O. House, Management of depression after stroke: a systematic review of pharmacological therapies. Stroke, 2005. 36(5): p. 1098-103.

32. Gabaldon, L., et al., Poststroke depression: importance of its detection and treatment. Cerebrovasc Dis, 2007. 24 Suppl 1: p. 181-8.

33. Desmond, D.W., et al., Frequency and clinical determinants of dementia after ischemic stroke. Neurology, 2000. 54(5): p. 1124-31.

34. Pohjasvaara, T., et al., Dementia three months after stroke. Baseline frequency and effect of different definitions of dementia in the Helsinki Stroke Aging Memory Study (SAM) cohort. Stroke, 1997. 28(4): p. 785-92.

35. Bowler, J.V. and V. Hachinski, Vascular Cognitive impairment: a new approach to vascular dementia, in Vascular Dementia. 1995. p. 357-376.

36. Pohjasvaara, T., et al., Evaluation of various methods of assessing symptoms of cognitive impairment and dementia. Alzheimer Dis Assoc Disord, 2001. 15(4): p. 184-93.

37. Rasquin, S.M., J. Lodder, and F.R. Verhey, The effect of different diagnostic criteria on the prevalence and incidence of post-stroke dementia. Neuroepidemiology, 2005. 24(4): p. 189-95.

38. Erkinjuntti, T., Vascular cognitive deterioration and stroke. Cerebrovasc Dis, 2007. 24 Suppl 1: p. 189-94.

39. Nys, G.M., et al., Cognitive disorders in acute stroke: prevalence and clinical determinants. Cerebrovasc Dis, 2007. 23(5-6): p. 408-16.

40. Fure, B., et al., Cognitive impairments in acute lacunar stroke. Acta Neurol Scand, 2006. 114(1): p. 17-22.

41. Lesniak, M., et al., Frequency and prognostic value of cognitive disorders in stroke patients. Dement Geriatr Cogn Disord, 2008. 26(4): p. 356-63.

42. Rasquin, S.M., et al., Cognitive functioning after stroke: a one-year follow-up study. Dement Geriatr Cogn Disord, 2004. 18(2): p. 138-44.

43. Llewellyn, D.J., et al., Framingham Stroke Risk Profile and poor cognitive function: a populationbased study. BMC Neurol, 2008. 8: p. 12.

44. van Zandvoort, M.J., et al., Early neuropsychological evaluation in patients with ischaemic stroke provides valid information. Clin Neurol Neurosurg, 2005. 107(5): p. 385-92.

45. Stephens, S., et al., Neuropsychological characteristics of mild vascular cognitive impairment and dementia after stroke. Int J Geriatr Psychiatry, 2004. 19(11): p. 1053-7.

46. Sachdev, P.S., et al., Progression of cognitive impairment in stroke patients. Neurology, 2004. 63(9): p. 1618-23.

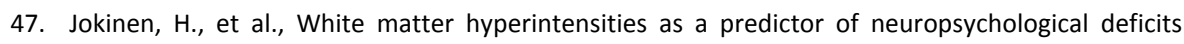
post-stroke. J Neurol Neurosurg Psychiatry, 2005. 76(9): p. 1229-33.

48. Moorhouse, P. and K. Rockwood, Vascular cognitive impairment: current concepts and clinical developments. Lancet Neurol, 2008. 7(3): p. 246-55. 


\section{References}

49. Hoffmann, M., F. Schmitt, and E. Bromley, Vascular cognitive syndromes: relation to stroke etiology and topography. Acta Neurol Scand, 2009.

50. Folstein, M.F., S.E. Folstein, and P.R. McHugh, "Mini-mental state". A practical method for grading the cognitive state of patients for the clinician. J Psychiatr Res, 1975. 12(3): p. 189-98.

51. Blake, H., et al., An evaluation of screening measures for cognitive impairment after stroke. Age Ageing, 2002. 31(6): p. 451-6.

52. Nys, G.M., et al., Restrictions of the Mini-Mental State Examination in acute stroke. Arch Clin Neuropsychol, 2005. 20(5): p. 623-9.

53. Agrell, B. and O. Dehlin, Mini mental state examination in geriatric stroke patients. Validity, differences between subgroups of patients, and relationships to somatic and mental variables. Aging (Milano), 2000. 12(6): p. 439-44.

54. Zwecker, M., et al., Mini-Mental State Examination, cognitive FIM instrument, and the Loewenstein Occupational Therapy Cognitive Assessment: relation to functional outcome of stroke patients. Arch Phys Med Rehabil, 2002. 83(3): p. 342-5.

55. Appelros, P., Characteristics of Mini-Mental State Examination 1 year after stroke. Acta Neurol Scand, 2005. 112(2): p. 88-92.

56. Kalaria, R.N. and C. Ballard, Overlap between pathology of Alzheimer disease and vascular dementia. Alzheimer Dis Assoc Disord, 1999. 13 Suppl 3: p. S115-23.

57. Viswanathan, A., W.A. Rocca, and C. Tzourio, Vascular risk factors and dementia: how to move forward? Neurology, 2009. 72(4): p. 368-74.

58. Purandare, N. and A. Burns, Cerebral emboli in the genesis of dementia. J Neurol Sci, 2009.

59. Oude Voshaar, R.C., et al., Asymptomatic spontaneous cerebral emboli and mood in a cohort of older people: a prospective study. Am J Geriatr Psychiatry, 2007. 15(12): p. 1057-60.

60. Bu, G., Apolipoprotein $E$ and its receptors in Alzheimer's disease: pathways, pathogenesis and therapy. Nat Rev Neurosci, 2009. 10(5): p. 333-44.

61. Hofman, A., et al., Atherosclerosis, apolipoprotein E, and prevalence of dementia and Alzheimer's disease in the Rotterdam Study. Lancet, 1997. 349(9046): p. 151-4.

62. McCarron, M.O., D. Delong, and M.J. Alberts, APOE genotype as a risk factor for ischemic cerebrovascular disease: a meta-analysis. Neurology, 1999. 53(6): p. 1308-11.

63. Richard, F., et al., Effect of the angiotensin l-converting enzyme I/D polymorphism on cognitive decline. The EVA Study Group. Neurobiol Aging, 2000. 21(1): p. 75-80.

64. Hardy, J., The amyloid hypothesis for Alzheimer's disease: a critical reappraisal. J Neurochem, 2009. 110(4): p. 1129-34.

65. Jack, C.R., Jr., et al., Hypothetical model of dynamic biomarkers of the Alzheimer's pathological cascade. Lancet Neurol. 9(1): p. 119-28.

66. Aben, I., Post-stroke depression. The need for a biopsychosocial approach. 2004.

67. Rasquin, S., Vascular Cognitive Impairment. Phenomenology, Course, Riskfactors. 2005.

68. Beck, A.T., et al., An Inventory for measuring depression. Archives of general psychiatry, 1961. 4: p. 561-571.

69. Zigmond, A.S. and R.P. Snaith, The Hospital Anxiety and Depression Scale. Acta Psychiatrica Scandinavica, 1983. 67(6): p. 361-370.

70. Derogatis, L.R., R.S. Lipman, and L. Covi, SCL-90: an outpatient psychiatric rating scale--preliminary report. Psychopharmacol Bull, 1973. 9(1): p. 13-28.

71. First, M.B., et al., Structured Clinical Interview for DSM-IV Axis I Disorders - Patient Edition (SCID$I / P$, Version 2.0). 1995, Biometrics Research Department, New York State Psychiatric Institute: New York (NY).

72. Hamilton, M., A rating scale for depression. Journal of neurology, neurosurgery and psychiatry, 1960. 23: p. 56-62. 
73. American Psychiatric Association, Diagnostic and Statistical Manual of Mental Disorders. 4th. ed. 1994, Washington (DC): American Psychiatric Press.

74. Aben, I., et al., Focal or generalized vascular brain damage and vulnerability to depression after stroke: a 1-year prospective follow-up study. Int Psychogeriatr, 2006. 18(1): p. 19-35.

75. Aben, I., et al., Personality and vulnerability to depression in stroke patients: a 1-year prospective follow-up study. Stroke, 2002. 33(10): p. 2391-5.

76. Aben, I., et al., Validity of the beck depression inventory, hospital anxiety and depression scale, SCL-90, and hamilton depression rating scale as screening instruments for depression in stroke patients. Psychosomatics, 2002. 43(5): p. 386-93.

77. Aben, I., et al., Depressie na een beroerte: signalering, diagnostiek en behandeling in de huisartsenpraktijk. Huisarts en Wetenschap, 2003: p. in press.

78. Rasquin, S.M., et al., Cognitive performance after first ever stroke related to progression of vascular brain damage: a 2 year follow up CT scan study. J Neurol Neurosurg Psychiatry, 2005. 76(8): p. 1075-9.

79. van Heugten, C., et al., Checklist for cognitive and emotional consequences following stroke (CLCE24): development, usability and quality of the self-report version. Clin Neurol Neurosurg, 2007. 109(3): p. 257-62.

80. Rasquin, S.M., J. Lodder, and F.R. Verhey, Predictors of reversible mild cognitive impairment after stroke: a 2-year follow-up study. J Neurol Sci, 2005. 229-230: p. 21-5.

81. Rasquin, S.M., et al., Predictive accuracy of $\mathrm{MCl}$ subtypes for Alzheimer's disease and vascular dementia in subjects with mild cognitive impairment: a 2-year follow-up study. Dement Geriatr Cogn Disord, 2005. 19(2-3): p. 113-9.

82. Rasquin, S.M., et al., Demographic and CT scan features related to cognitive impairment in the first year after stroke. J Neurol Neurosurg Psychiatry, 2004. 75(11): p. 1562-7.

83. Teunisse, S. and M.M. Derix, [Measurement of activities of daily living in patients with dementia living at home: development of a questionnaire ]. Tijdschr Gerontol Geriatr, 1991. 22(2): p. 53-9.

84. Roman, G.C., et al., Vascular dementia: diagnostic criteria for research studies. Report of the NINDS-AIREN International Workshop [see comments]. Neurology, 1993. 43(2): p. 250-60.

85. Hochstenbach, J., The cognitive, emotional and behavioural consequences of stroke. 1999, Katholieke Universiteit Nijmegen: Nijmegen.

86. Williams, L.S., S.S. Ghose, and R.W. Swindle, Depression and other mental health diagnoses increase mortality risk after ischemic stroke. Am J Psychiatry, 2004. 161(6): p. 1090-5.

87. Andersen, G., Incidence of post-stroke depression during the first year in a large unselected stroke population determined using a valis standardized rating scale. Acta Psychiatr Scand, 1994. 90: p. 190-195.

88. Lipsey, J., Phenomenological comparison of poststroke depression and functional depression. Am J Psychiatr, 1986. 143: p. 527-529.

89. Fedoroff, J.P., et al., Phenomenological comparisons of major depression following stroke, myocardial infarction or spinal cord lesions. J Affect Disord, 1991. 22(1-2): p. 83-9.

90. Coster, L.d., The sensetivity of somatic symptoms in post-stroke depression: a discriminant analytic approach. Int J Geriatr Psychiatry, 2005. 20: p. 358-362.

91. Rasquin, S.M., et al., [Checklist for the detection of cognitive and emotional consequences after stroke (CLCE-24)]. Tijdschr Gerontol Geriatr, 2006. 37(3): p. 112-6.

92. Carson, A.J., et al., Depression after stroke and lesion location: a systematic review. Lancet, 2000. 356(9224): p. 122-6.

93. De Groot, J.C., et al., Cerebral white matter lesions and depressive symptoms in elderly adults. Arch Gen Psychiatry, 2000. 57(11): p. 1071-6.

94. Burvill, P., et al., Risk factors for post-stroke depression. Int J Geriatr Psychiatry, 1997. 12(2): p. 219-26. 


\section{References}

95. Herrmann, N., et al., The Sunnybrook Stroke Study: a prospective study of depressive symptoms and functional outcome. Stroke, 1998. 29(3): p. 618-624.

96. Hayee, M.A., et al., Depression after stroke-analysis of 297 stroke patients. Bangladesh Med Res Counc Bull, 2001. 27(3): p. 96-102.

97. Bush, B.A., Major life events as risk factors for post-stroke depression. Brain Injury, 1999. 13(2): p. 131-137.

98. Beekman, A.T., et al., Depression in survivors of stroke: a community-based study of prevalence, risk factors and consequences. Soc Psychiatry Psychiatr Epidemiol, 1998. 33(10): p. 463-470.

99. $\mathrm{CBO}$, Richtlijn behandeling van neuropsychiatrische gevolgen van niet-aangeboren hersenletsel. 2007.

100. Hackett, M.L., et al., Interventions for preventing depression after stroke. Cochrane Database Syst Rev, 2008(3): p. CD003689.

101. Paolucci, S., et al., Poststroke depression and its role in rehabilitation of inpatients. Arch Phys Med Rehabil, 1999. 80(9): p. 985-90.

102. Pohjasvaara, T., et al., Post-stroke depression, executive dysfunction and functional outcome. Eur J Neurol, 2002. 9(3): p. 269-75.

103. Rasquin, S., J. Lodder, and F. Verhey, The association between psychiatric and cognitive symptoms after stroke: a prospective study. Cerebrovasc Dis, 2005. 19(5): p. 309-16.

104. Bhogal, S.K., et al., Lesion location and poststroke depression: systematic review of the methodological limitations in the literature. Stroke, 2004. 35(3): p. 794-802.

105. D'Agostino, R.B., Sr., et al., General cardiovascular risk profile for use in primary care: the Framingham Heart Study. Circulation, 2008. 117(6): p. 743-53.

106. Special report from the National Institute of Neurological Disorders and Stroke. Classification of cerebrovascular diseases III. Stroke, 1990. 21(4): p. 637-76.

107. Boon, A., et al., Silent brain infarcts in 755 consecutive patients with a first-ever supratentorial ischemic stroke. Relationship with index-stroke subtype, vascular risk factors, and mortality. Stroke, 1994. 25(12): p. 2384-90.

108. Herrmann, C., International experiences with the Hospital Anxiety and Depression Scale -a review of validation data and clinical results. Journal of Psychosomatic Research, 1997. 42(1): p. 17-41.

109. Richter, P., et al., On the validity of the Beck Depression Inventory. A review. Psychopathology, 1998. 31(3): p. 160-8.

110. Arrindell, W.A. and J.H.M. Ettema, Symptom Checklist [Handleiding bij een multidimensionele psychopathologie-indicator]. 2003, Lisse: Swets \& Zeitlinger B.V.

111. Strik, J.J., et al., Sensitivity and specificity of observer and self-report questionnaires in major and minor depression following myocardial infarction. Psychosomatics, 2001. 42(5): p. 423-8.

112. Lampe, I.K. and T.J. Heeren, Is apathy in late-life depressive illness related to age-at-onset, cognitive function or vascular risk? Int Psychogeriatr, 2004. 16(4): p. 481-6.

113. Brodaty, $\mathrm{H}_{\text {., et }}$ al., Frequency and clinical, neuropsychological and neuroimaging correlates of apathy following stroke - the Sydney Stroke Study. Psychol Med, 2005. 35(12): p. 1707-16.

114. Starkstein, S.E., et al., On the overlap between apathy and depression in dementia. J Neurol Neurosurg Psychiatry, 2005. 76(8): p. 1070-4.

115. Richard, I.H., Apathy does not equal depression in Parkinson disease: why we should care. Neurology, 2006. 67(1): p. 10-1.

116. Starkstein, S.E. and A.F. Leentjens, The nosological position of apathy in clinical practice. J Neurol Neurosurg Psychiatry, 2008.

117. Angelelli, P., et al., Development of neuropsychiatric symptoms in poststroke patients: a crosssectional study. Acta Psychiatr Scand, 2004. 110(1): p. 55-63.

118. Tateno, A., M. Kimura, and R.G. Robinson, Phenomenological characteristics of poststroke depression: early- versus late-onset. Am J Geriatr Psychiatry, 2002. 10(5): p. 575-82. 
119. Gainotti, G., A. Azzoni, and C. Marra, Frequency, phenomenology and anatomical-clinical correlates of major post-stroke depression. Br J Psychiatry, 1999. 175: p. 163-7.

120. Beekman, A.T., J.R. Copeland, and M.J. Prince, Review of community prevalence of depression in later life. Br J Psychiatry, 1999. 174: p. 307-11.

121. de Jonge, P., et al., Depressive symptoms in elderly patients after a somatic illness event: prevalence, persistence, and risk factors. Psychosomatics, 2006. 47(1): p. 33-42.

122. Lyness, J.M., et al., Cerebrovascular risk factors and 1-year depression outcome in older primary care patients. American Journal of Psychiatry, 2000. 157(9): p. 1499-1501 FTXT: HighWire http://ajp psychiatryonline org/cgi/content/full/157/9/1499.

123. Mast, B.T., et al., Longitudinal support for the relationship between vascular risk factors and latelife depressive symptoms. Am J Geriatr Psychiatry, 2004. 12(1): p. 93-101.

124. Denollet, J., et al., Personality as independent predictor of long-term mortality in patients with coronary heart disease. Lancet, 1996. 347(8999): p. 417-21.

125. Strik, J.J., A. Honig, and M. Maes, Depression and myocardial infarction: relationship between heart and mind. Prog Neuropsychopharmacol Biol Psychiatry, 2001. 25(4): p. 879-92.

126. Johnson, G., et al., Screening instruments for depression and anxiety following stroke: experience in the Perth community stroke study. Acta Psychiatr Scand, 1995. 91(4): p. 252-7.

127. Fure, B., et al., Emotional symptoms in acute ischemic stroke. Int J Geriatr Psychiatry, 2006. 21(4): p. 382-7.

128. Johnston, M., B. Pollard, and P. Hennessey, Construct validation of the hospital anxiety and depression scale with clinical populations. J Psychosom Res, 2000. 48(6): p. 579-84.

129. Gainotti, G., et al., The Post-Stroke Depression Rating Scale: a test specifically devised to investigate affective disorders of stroke patients. J Clin Exp Neuropsychol, 1997. 19(3): p. 340-56.

130. Morrison, V., et al., Anxiety and depression 3 years following stroke: Demographic, clinical, and psychological predictors. J Psychosom Res, 2005. 59(4): p. 209-13.

131. Sharpe, M., et al., Depressive disorders in long-term survivors of stroke. Associations with demographic and social factors, functional status, and brain lesion volume. British Journal of Psychiatry, 1994. 164(3): p. 380-386.

132. Carota, A., et al., A prospective study of predictors of poststroke depression. Neurology, 2005. 64(3): p. 428-33.

133. Andersen, G., et al., Risk factors for post-stroke depression. Acta Psychiatr Scand, 1995. 92(3): p. 193-198.

134. Gaete, J.M. and J. Bogousslavsky, Post-stroke depression. Expert Rev Neurother, 2008. 8(1): p. 7592.

135. Whyte, E.M., et al., Depression after stroke: a prospective epidemiological study. J Am Geriatr Soc, 2004. 52(5): p. 774-8.

136. Narushima, K. and R.G. Robinson, The effect of early versus late antidepressant treatment on physical impairment associated with poststroke depression: is there a time-related therapeutic window? J Nerv Ment Dis, 2003. 191(10): p. 645-52.

137. Paranthaman, R. and R.C. Baldwin, Treatment of psychiatric syndromes due to cerebrovascular disease. Int Rev Psychiatry, 2006. 18(5): p. 453-70.

138. Costa, P.T., Jr. and R.R. McCrae, The NEO Personality Inventory Manual. 1985, Psychological Assessment Resources Inc.: Odessa, Florida.

139. Hoekstra, H.A., J. Ormel, and F. De Fruyt, NEO Personality Questionnaires NEO-PI-R, NEO-FFI, Manual. 1996, Lisse: Swets \& Zeitlinger B.V.

140. Enns, M.W. and B.J. Cox, Personality dimensions and depression: Review and commentary. Canadian Journal of Psychiatry, 1997. 42(3): p. 274-284.

141. Morris, P.L., R.G. Robinson, and B. Raphael, Prevalence and course of depressive disorders in hospitalized stroke patients. Int J Psychiatry Med, 1990. 20(4): p. 349-64. 


\section{References}

142. Burvill, P.W., et al., Prevalence of depression after stroke: the Perth Community Stroke Study. Br J Psychiatry, 1995. 166(3): p. 320-7.

143. Hackett, M.L. and C.S. Anderson, Frequency, management, and predictors of abnormal mood after stroke: the Auckland Regional Community Stroke (ARCOS) study, 2002 to 2003. Stroke, 2006. 37(8): p. 2123-8.

144. Andersen, G., K. Vestergaard, and L. Lauritzen, Effective treatment of poststroke depression with the selective serotonin reuptake inhibitor citalopram. Stroke, 1994. 25(6): p. 1099-104.

145. Townend, B.S., et al., Longitudinal prevalence and determinants of early mood disorder poststroke. J Clin Neurosci, 2007. 14(5): p. 429-34.

146. Rockwood, K., Vascular cognitive impairment and vascular dementia. J Neurol Sci, 2002. 203204(C): p. 23-7.

147. Kauhanen, M., et al., Poststroke depression correlates with cognitive impairment and neurological deficits. Stroke, 1999. 30(9): p. 1875-80.

148. Dam, H., Depression in stroke patients 7 years following stroke. Acta Psychiatr Scand, 2001. 103(4): p. 287-93.

149. Li, Y., J.S. Meyer, and J. Thornby, Depressive symptoms among cognitively normal versus cognitively impaired elderly subjects. Int J Geriatr Psychiatry, 2001. 16(5): p. 455-61.

150. Hajjar, I., et al., A Novel Aging Phenotype of Slow Gait, Impaired Executive Function, and Depressive Symptoms: Relationship to Blood Pressure and Other Cardiovascular Risks. J Gerontol A Biol Sci Med Sci, 2009.

151. Petersen, R.C., Mild cognitive impairment as a diagnostic entity. J Intern Med, 2004. 256(3): p. 183-94.

152. Dotson, V.M., S.M. Resnick, and A.B. Zonderman, Differential association of concurrent, baseline, and average depressive symptoms with cognitive decline in older adults. Am J Geriatr Psychiatry, 2008. 16(4): p. 318-30.

153. Wilson, R.S., et al., Depressive symptoms and cognitive decline in a community population of older persons. J Neurol Neurosurg Psychiatry, 2004. 75(1): p. 126-9.

154. Starkstein, S.E., et al., A prospective longitudinal study of apathy in Alzheimer's disease. J Neurol Neurosurg Psychiatry, 2006. 77(1): p. 8-11.

155. Butters, M.A., et al., The nature and determinants of neuropsychological functioning in late-life depression. Arch Gen Psychiatry, 2004. 61(6): p. 587-95.

156. Elderkin-Thompson, V., et al., Executive dysfunction and memory in older patients with major and minor depression. Arch Clin Neuropsychol, 2006. 21(7): p. 669-76.

157. Sanders, M.L., et al., Cerebrovascular risk factors, executive dysfunction, and depression in older primary care patients. Am J Geriatr Psychiatry, 2006. 14(2): p. 145-52.

158. Naarding, P., et al., Depression in vascular dementia. Int J Geriatr Psychiatry, 2003. 18(4): p. 32530.

159. Smith, P.J., et al., Cerebrovascular risk factors, vascular disease, and neuropsychological outcomes in adults with major depression. Psychosom Med, 2007. 69(6): p. 578-86.

160. Zanetti, M., et al., Mild cognitive impairment subtypes and vascular dementia in communitydwelling elderly people: a 3-year follow-up study. J Am Geriatr Soc, 2006. 54(4): p. 580-6.

161. Braaten, A.J., et al., Neurocognitive differential diagnosis of dementing diseases: Alzheimer's Dementia, Vascular Dementia, Frontotemporal Dementia, and Major Depressive Disorder. Int J Neurosci, 2006. 116(11): p. 1271-93.

162. Downhill, J.E., Jr. and R.G. Robinson, Longitudinal assessment of depression and cognitive impairment following stroke. J Nerv Ment Dis, 1994. 182(8): p. 425-31.

163. Saczynski, J.S., et al., Cerebral infarcts and cognitive performance: importance of location and number of infarcts. Stroke, 2009. 40(3): p. 677-82. 
164. Murata, Y., M. Kimura, and R.G. Robinson, Does cognitive impairment cause post-stroke depression? Am J Geriatr Psychiatry, 2000. 8(4): p. 310-7.

165. Naismith, S.L., et al., Cognitive training in affective disorders improves memory: A preliminary study using the NEAR approach. J Affect Disord, 2009.

166. Butters, M.A., et al., Changes in cognitive functioning following treatment of late-life depression. Am J Psychiatry, 2000. 157(12): p. 1949-54.

167. Alexopoulos, G.S., et al., Clinical presentation of the "depression-executive dysfunction syndrome" of late life. Am J Geriatr Psychiatry, 2002. 10(1): p. 98-106.

168. Hudon, C., S. Belleville, and S. Gauthier, The association between depressive and cognitive symptoms in amnestic mild cognitive impairment. Int Psychogeriatr, 2008. 20(4): p. 710-23.

169. Mast, B.T., et al., Risk factors for geriatric depression: the importance of executive functioning within the vascular depression hypothesis. J Gerontol A Biol Sci Med Sci, 2004. 59(12): p. 1290-4.

170. de Koning, I., et al., The CAMCOG: a useful screening instrument for dementia in stroke patients. Stroke, 1998. 29(10): p. 2080-6.

171. Verhey, F.R.J., et al., Cross-sectional comparison of the Cambridge Cognitive Examnination revised: The CAMCOG-R. Age and Aging, 2003. 32: p. 1-7.

172. Houx, P.J., J. Jolles, and F.W. Vreeling, Stroop interference: aging effects assessed with the Stroop Color-Word Test. Experimental Aging Research, 1993. 19(204-224).

173. Reitan, R.M., Validity of the Trail Making Test as an indication of organic brain damage. Perceptual and motor skills, 1985. 8: p. 271-276.

174. Cui, X., et al., Does depression precede or follow executive dysfunction? Outcomes in older primary care patients. Am J Psychiatry, 2007. 164(8): p. 1221-8.

175. Nys, G.M., et al., Early depressive symptoms after stroke: neuropsychological correlates and lesion characteristics. J Neurol Sci, 2005. 228(1): p. 27-33.

176. Thomas, A.J. and J.T. O'Brien, Depression and cognition in older adults. Curr Opin Psychiatry, 2008. 21(1): p. 8-13.

177. Lockwood, K.A., G.S. Alexopoulos, and W.G. van Gorp, Executive dysfunction in geriatric depression. Am J Psychiatry, 2002. 159(7): p. 1119-26.

178. O'Brien, J.T., et al., Vascular cognitive impairment. Lancet Neurol, 2003. 2(2): p. 89-98.

179. Verdelho, A., et al., Depressive symptoms after stroke and relationship with dementia: A threeyear follow-up study. Neurology, 2004. 62(6): p. 905-11.

180. Narushima, K., et al., Does cognitive recovery after treatment of poststroke depression last? A 2year follow-up of cognitive function associated with poststroke depression. Am J Psychiatry, 2003. 160(6): p. 1157-62.

181. Robinson, R.G., Neuropsychiatric consequences of stroke. Annu Rev Med, 1997. 48: p. 217-29.

182. Alexopoulos, G.S., et al., Executive dysfunction and long-term outcomes of geriatric depression. Arch Gen Psychiatry, 2000. 57(3): p. 285-90.

183. Hama, S., et al., Depression or apathy and functional recovery after stroke. Int J Geriatr Psychiatry, 2007. 22(10): p. 1046-51.

184. Steffens, D.C., W.D. Taylor, and K.R. Krishnan, Progression of subcortical ischemic disease from vascular depression to vascular dementia. Am J Psychiatry, 2003. 160(10): p. 1751-6.

185. Saxena, S.K., et al., Is improvement in impaired cognition and depressive symptoms in post-stroke patients associated with recovery in activities of daily living? Acta Neurol Scand, 2007. 115(5): p. 339-46.

186. Scuteri, A., et al., Age-related changes in cognitive domains. A population-based study. Aging Clin Exp Res, 2005. 17(5): p. 367-73.

187. Patel, M., et al., Natural history of cognitive impairment after stroke and factors associated with its recovery. Clin Rehabil, 2003. 17(2): p. 158-66. 


\section{References}

188. Heruti, R.J., et al., Rehabilitation outcome of elderly patients after a first stroke: effect of cognitive status at admission on the functional outcome. Arch Phys Med Rehabil, 2002. 83(6): p. 742-9.

189. Borjesson-Hanson, A., D. Gustafson, and I. Skoog, Five-year mortality in relation to dementia and cognitive function in 95-year-olds. Neurology, 2007. 69(22): p. 2069-75.

190. Denti, L., M. Agosti, and M. Franceschini, Outcome predictors of rehabilitation for first stroke in the elderly. Eura Medicophys, 2007.

191. Rabadi, M.H., et al., Cognitively impaired stroke patients do benefit from admission to an acute rehabilitation unit. Arch Phys Med Rehabil, 2008. 89(3): p. 441-8.

192. Tombaugh, T.N. and N.J. McIntyre, The mini-mental state examination: a comprehensive review. J Am Geriatr Soc, 1992. 40(9): p. 922-35.

193. Dick, J.P., et al., Mini-mental state examination in neurological patients. J Neurol Neurosurg Psychiatry, 1984. 47(5): p. 496-9.

194. O'Sullivan, M., R.G. Morris, and H.S. Markus, Brief cognitive assessment for patients with cerebral small vessel disease. J Neurol Neurosurg Psychiatry, 2005. 76(8): p. 1140-5.

195. Anthony, J.C., et al., Limits of the 'Mini-Mental State' as a screening test for dementia and delirium among hospital patients. Psychol Med, 1982. 12(2): p. 397-408.

196. Jolles, J., et al., The Maastricht Aging Study: Determinants of cognitive aging. 1995, Maastricht: Neuropsych Publishers.

197. Petersen, R.C., et al., Mild cognitive impairment: clinical characterization and outcome. Arch Neurol, 1999. 56(3): p. 303-8.

198. Feher, E.P., et al., Establishing the limits of the Mini-Mental State. Examination of 'subtests'. Arch Neurol, 1992. 49(1): p. 87-92.

199. Kukull, W.A., et al., The Mini-Mental State Examination score and the clinical diagnosis of dementia. J Clin Epidemiol, 1994. 47(9): p. 1061-7.

200. Popovic, I.M., V. Seric, and V. Demarin, Mild cognitive impairment in symptomatic and asymptomatic cerebrovascular disease. J Neurol Sci, 2007. 257(1-2): p. 185-93.

201. Crum, R.M., et al., Population-based norms for the Mini-Mental State Examination by age and educational level. Jama, 1993. 269(18): p. 2386-91.

202. MacKenzie, D.M., et al., Brief cognitive screening of the elderly: a comparison of the Mini-Mental State Examination (MMSE), Abbreviated Mental Test (AMT) and Mental Status Questionnaire (MSQ). Psychol Med, 1996. 26(2): p. 427-30.

203. Suhr, J.A. and J. Grace, Brief cognitive screening of right hemisphere stroke: relation to functional outcome. Arch Phys Med Rehabil, 1999. 80(7): p. 773-6.

204. Appelros, P. and A.G. Andersson, Changes in Mini Mental State Examination score after stroke: lacunar infarction predicts cognitive decline. Eur J Neurol, 2006. 13(5): p. 491-5.

205. Nelson, A., B.S. Fogel, and D. Faust, Bedside cognitive screening instruments. A critical assessment. J Nerv Ment Dis, 1986. 174(2): p. 73-83.

206. Riepe, M.W., et al., Screening for cognitive impairment in patients with acute stroke. Dement Geriatr Cogn Disord, 2004. 17(1-2): p. 49-53.

207. del Ser, T., et al., Evolution of cognitive impairment after stroke and risk factors for delayed progression. Stroke, 2005. 36(12): p. 2670-5.

208. Solfrizzi, V., et al., Vascular risk factors, incidence of $\mathrm{MCl}$, and rates of progression to dementia. Neurology, 2004. 63(10): p. 1882-91.

209. Rockwood, K., et al., Spectrum of disease in vascular cognitive impairment. Neuroepidemiology, 1999. 18(5): p. 248-54.

210. Gorelick, P.B. and J.V. Bowler, Advances in vascular cognitive impairment 2007. Stroke, 2008. 39(2): p. 279-82.

211. Jellinger, K.A., The pathology of "vascular dementia": a critical update. J Alzheimers Dis, 2008. 14(1): p. 107-23. 
212. Helzner, E.P., et al., Contribution of vascular risk factors to the progression in Alzheimer disease. Arch Neurol, 2009. 66(3): p. 343-8.

213. O'Brien, J. and S. Lilienfeld, Relevant clinical outcomes in probable vascular dementia and Alzheimer's disease with cerebrovascular disease. J Neurol Sci, 2002. 203-204: p. 41-8.

214. Launer, L.J., et al., AD brain pathology: vascular origins? Results from the HAAS autopsy study. Neurobiol Aging, 2008. 29(10): p. 1587-90.

215. Hsiung, G.Y., A.D. Sadovnick, and H. Feldman, Apolipoprotein E epsilon4 genotype as a risk factor for cognitive decline and dementia: data from the Canadian Study of Health and Aging. Cmaj, 2004. 171(8): p. 863-7.

216. Knopman, D.S., et al., Fourteen-year longitudinal study of vascular risk factors, APOE genotype, and cognition: the ARIC MRI Study. Alzheimers Dement, 2009. 5(3): p. 207-14.

217. Kolsch, H., et al., ACE I/D polymorphism is a risk factor of Alzheimer's disease but not of vascular dementia. Neurosci Lett, 2005. 377(1): p. 37-9.

218. Morovic, S., et al., Vascular characteristics of patients with dementia. J Neurol Sci, 2009.

219. Federoff, H.J., Alzheimer's disease: reducing the burden with ApoE2. Gene Ther, 2005. 12(13): p. 1019-29.

220. Mortimer, J.A., D.A. Snowdon, and W.R. Markesbery, The effect of APOE-epsilon4 on dementia is mediated by Alzheimer neuropathology. Alzheimer Dis Assoc Disord, 2009. 23(2): p. 152-7.

221. Miners, J.S., et al., Angiotensin-converting enzyme (ACE) levels and activity in Alzheimer's disease, and relationship of perivascular ACE-1 to cerebral amyloid angiopathy. Neuropathol Appl Neurobiol, 2008. 34(2): p. 181-93.

222. Hassan, A., et al., Angiotensin converting enzyme insertion/deletion genotype is associated with leukoaraiosis in lacunar syndromes. J Neurol Neurosurg Psychiatry, 2002. 72(3): p. 343-6.

223. Szolnoki, Z., et al., Coexistence of angiotensin II type-1 receptor A1166C and angiotensinconverting enzyme $D / D$ polymorphism suggests susceptibility for small-vessel-associated ischemic stroke. Neuromolecular Med, 2006. 8(3): p. 353-60.

224. Bartres-Faz, D., et al., Angiotensin I converting enzyme polymorphism in humans with ageassociated memory impairment: relationship with cognitive performance. Neurosci Lett, 2000. 290(3): p. 177-80.

225. Purandare, N., et al., Deletion/insertion polymorphism of the angiotensin-converting enzyme gene and white matter hyperintensities in dementia: A pilot study. J Am Geriatr Soc, 2006. 54(9): p. 1395-400.

226. Vardy, E.R., et al., Plasma angiotensin-converting enzyme in Alzheimer's disease. J Alzheimers Dis, 2009. 16(3): p. 609-18.

227. Baum, L., et al., Polymorphisms and vascular cognitive impairment after ischemic stroke. J Geriatr Psychiatry Neurol, 2007. 20(2): p. 93-9.

228. Liu, H., et al., Association of ACE I/D Gene Polymorphism with Vascular Dementia: A MetaAnalysis. J Geriatr Psychiatry Neurol, 2008.

229. Arpa, A., et al., Apolipoprotein E, angiotensin-converting enzyme and alpha-1-antichymotrypsin genotypes are not associated with post-stroke dementia. J Neurol Sci, 2003. 210(1-2): p. 77-82.

230. Slooter, A.J., et al., Apolipoprotein E epsilon4 and the risk of dementia with stroke. A populationbased investigation. Jama, 1997. 277(10): p. 818-21.

231. Bowler, J.V., Vascular cognitive impairment. J Neurol Neurosurg Psychiatry, 2005. 76 Suppl 5: p. v35-44.

232. Bowler, J.V., Modern concept of vascular cognitive impairment. Br Med Bull, 2007. 83: p. 291-305.

233. Van der Elst, W., et al., The Stroop color-word test: influence of age, sex, and education; and normative data for a large sample across the adult age range. Assessment, 2006. 13(1): p. 62-79.

234. Brand, P.A. and J. Jolles, Learning and retrieval rate of words presented auditorialy and visualy. The Journal of General Psychology, 1985. 112: p. 201-210. 


\section{References}

235. Van der Elst, W., et al., Rey's verbal learning test: normative data for 1855 healthy participants aged 24-81 years and the influence of age, sex, education, and mode of presentation. J Int Neuropsychol Soc, 2005. 11(3): p. 290-302.

236. Landis, J.R. and G.G. Koch, The measurement of observer agreement for categorical data. Biometrics, 1977. 33(1): p. 159-74.

237. Snijders, T. and R. Bosker, Multilevel analysis. An introduction to basic and advanced multilevel modeling. London: Sage, 1999.

238. Traykov, L., et al., Apolipoprotein E phenotypes in demented and cognitively impaired patients with and without cerebrovascular disease. Eur J Neurol, 1999. 6(4): p. 415-21.

239. Frank, A., et al., APOE genotype in cerebrovascular disease and vascular dementia. J Neurol Sci, 2002. 203-204: p. 173-6.

240. Zhu, L., et al., Incidence of dementia in relation to stroke and the apolipoprotein E epsilon4 allele in the very old. Findings from a population-based longitudinal study. Stroke, 2000. 31(1): p. 53-60.

241. Tabet, N., R. Quinn, and A. Klugman, Prevalence and cognitive impact of cerebrovascular findings in Alzheimer's disease: a retrospective, naturalistic study. Int J Clin Pract, 2009. 63(2): p. 338-45.

242. Lewis, H., et al., Quantification of Alzheimer pathology in ageing and dementia: age-related accumulation of amyloid-beta(42) peptide in vascular dementia. Neuropathol Appl Neurobiol, 2006. 32(2): p. 103-18.

243. Burlinson, S., et al., Effect of apolipoprotein E status on clinical features of dementia. Int J Geriatr Psychiatry, 1998. 13(3): p. 177-85.

244. Orsitto, G., et al., Apolipoprotein E genotypes in hospitalized elderly patients with vascular dementia. Dement Geriatr Cogn Disord, 2007. 23(5): p. 327-33.

245. Gdovinova, Z., V. Habalova, and Z. Novosadova, Polymorphism of apolipoproteine E in relation with Alzheimer and vascular dementia. Cell Mol Neurobiol, 2006. 26(7-8): p. 1219-24.

246. Baum, L., et al., Apolipoprotein E epsilon4 allele is associated with vascular dementia. Dement Geriatr Cogn Disord, 2006. 22(4): p. 301-5.

247. Slooter, A.J., et al., Risk estimates of dementia by apolipoprotein E genotypes from a populationbased incidence study: the Rotterdam Study. Arch Neurol, 1998. 55(7): p. 964-8.

248. Liu, Y., et al., Apolipoprotein E polymorphism and acute ischemic stroke: a diffusion- and perfusion-weighted magnetic resonance imaging study. J Cereb Blood Flow Metab, 2002. 22(11): p. 1336-42.

249. Engelborghs, S., et al., Prospective Belgian study of neurodegenerative and vascular dementia: APOE genotype effects. J Neurol Neurosurg Psychiatry, 2003. 74(8): p. 1148-51.

250. Davidson, Y., et al., Apolipoprotein E epsilon4 allele frequency and age at onset of Alzheimer's disease. Dement Geriatr Cogn Disord, 2007. 23(1): p. 60-6.

251. Szolnoki, Z., et al., Specific APO E genotypes in combination with the ACE D/D or MTHFR 677TT mutation yield an independent genetic risk of leukoaraiosis. Acta Neurol Scand, 2004. 109(3): p. 222-7.

252. Gustafson, D.R., et al., The ACE Insertion Deletion polymorphism relates to dementia by metabolic phenotype, APOEvarepsilon4, and age of dementia onset. Neurobiol Aging, 2008.

253. Kim, Y., et al., Sequence variants of ACE, AGT, AT1R, and PAI-1 as genetic risk factors for vascular dementia. Neurosci Lett, 2006. 401(3): p. 276-9.

254. Wang, H.K., et al., Apolipoprotein E, angiotensin-converting enzyme and kallikrein gene polymorphisms and the risk of Alzheimer's disease and vascular dementia. J Neural Transm, 2006. 113(10): p. 1499-509.

255. Jokinen, H., et al., Cognitive profile of subcortical ischaemic vascular disease. J Neurol Neurosurg Psychiatry, 2006. 77(1): p. 28-33.

256. Loewenstein, D.A., et al., Cognitive profiles in Alzheimer's disease and in mild cognitive impairment of different etiologies. Dement Geriatr Cogn Disord, 2006. 21(5-6): p. 309-15. 
257. Busse, A., M.C. Angermeyer, and S.G. Riedel-Heller, Progression of mild cognitive impairment to dementia: a challenge to current thinking. Br J Psychiatry, 2006. 189: p. 399-404.

258. Sachdev, P.S., et al., The determinants and longitudinal course of post-stroke mild cognitive impairment. J Int Neuropsychol Soc, 2009. 15(6): p. 915-23.

259. Schepers, V., et al., Prediction of depressive symptoms up to three years post-stroke. J Rehabil Med, 2009. 41(11): p. 930-5.

260. Nys, G.M., et al., Early cognitive impairment predicts long-term depressive symptoms and quality of life after stroke. J Neurol Sci, 2006.

261. Flicker, L., Vascular factors in geriatric psychiatry: time to take a serious look. Curr Opin Psychiatry, 2008. 21(6): p. 551-4.

262. Austin, M.P., P. Mitchell, and G.M. Goodwin, Cognitive deficits in depression: possible implications for functional neuropathology. Br J Psychiatry, 2001. 178: p. 200-6.

263. Staekenborg, S.S., et al., Behavioural and psychological symptoms in vascular dementia; differences between small and large vessel disease. J Neurol Neurosurg Psychiatry, 2009.

264. Strozyk, D., et al., Contribution of vascular pathology to the clinical expression of dementia. Neurobiol Aging, 2008.

265. Mayo, N.E., et al., A longitudinal view of apathy and its impact after stroke. Stroke, 2009. 40(10): p. 3299-307.

266. Oksala, N.K., et al., Cognitive impairment predicts poststroke death in long-term follow-up. J Neurol Neurosurg Psychiatry, 2009. 80(11): p. 1230-5.

267. Merriman, C., P. Norman, and J. Barton, Psychological correlates of PTSD symptoms following stroke. Psychol Health Med, 2007. 12(5): p. 592-602.

268. Howard, K.L. and C.M. Filley, Advances in genetic testing for Alzheimer's disease. Rev Neurol Dis, 2009. 6(1): p. 26-32.

269. Kalaria, R.N., Arteriosclerosis, apolipoprotein E, and Alzheimer's disease. Lancet, 1997. 349(9059): p. 1174.

270. Bella, R., et al., Clinical Presentation and Outcome of Geriatric Depression in Subcortical Ischemic Vascular Disease. Gerontology, 2009.

271. Shah, K., et al., Does use of antihypertensive drugs affect the incidence or progression of dementia? A systematic review. Am J Geriatr Pharmacother, 2009. 7(5): p. 250-61.

272. Kalmijn, S., et al. , Cerebrovascular disease, the Apolipoprotein e4 allele, and cognitive decline in a community-based study of elderly men. Stroke, 1996. 27:p. 2230-2235.

273. Ballard, C.G., et al., APOE epsilon4 and cognitive decline in older stroke patients with early cognitive impairment. Neurology, 2004. 63;p. 1399-1402.

274. Wagle, J., et al. Association between APOE epsilon4 and cognitive impairment after stroke. Dement Geriatr Cogn Disord, 2009. 27:p. 525-533.

275. Klages, J.D., et al., APOE genotype, vascular risk factors, memory test perfoemance and the fiveyear risk of vascular cognitive impairment or Alzheimer's disease. Dement Geriatr Cogn Disord, 2005. 20:p. 292-297

276. Reitz, C., et al., Stroek and memory performancein elderly persons without dementia. Arch Neurol, 2006. 63:p. 571-576

277. Rowan, E., et al., Impact of hypertension and Apolipoprotein e4 on poststroke cognition in subjects $>75$ years of age. Stroke, 2005.36:p. 1864-1868. 

Dankwoord 
Mijn komst naar Maastricht halverwege mijn opleiding tot neuroloog was een mooie gelegenheid om me verder te ontwikkelen op wetenschappelijk terrein. Ik wilde me toeleggen op de gedragsneurologie en mij daar begeven waar de grenzen tussen neurologie en psychiatrie niet meer zo duidelijk zijn. En daar lag een mooi project voor mij klaar, waaruit dit proefschrift is voortgekomen. Een dergelijk proces naast het vervolgen van een opleiding en het goed willen uitvoeren van klinisch werk vergt veel. Niet alleen heb ik wetenschappelijk veel geleerd, maar ook persoonlijk heb ik veel opgestoken van de mensen met wie ik heb samengewerkt. Een aantal mensen wil ik in het bijzonder danken hiervoor.

Mijn twee promotoren en mijn co-promotor prof. dr. F. Verhey, prof. dr. M. Limburg en dr. S. Rasquin. Wat heb ik veel van jullie geleerd! Bedankt voor jullie begeleiding.

Beste Frans, mijn grote dank voor de intensieve begeleiding. Hoe vaak ik ook niet vastgelopen en in verwarring een beroep op je deed, altijd wist je me in een mum weer op het pad te trekken door je enthousiasme, je immer positieve instelling en je pragmatisme. Ik heb veel geleerd van en zeer grote bewondering voor jouw veelzijdigheid als clinicus, wetenschapper en manager / leidinggevende. Je interesse in mijn persoon waardeer ik enorm. Onze gesprekken over 'gedrag', het vak 'zenuwarts' en je visie op het brein in het bijzonder ga ik missen. Ik hoop dat ik in de toekomst nog veel met je van doen mag hebben, aangezien we inderdaad naar dezelfde film kijken, zij het door een andere bril.

Beste Martien, bedankt voor je interesse in het verloop van mijn onderzoek en je adviezen. In het bijzonder mijn dank voor je nauwkeurige en kritische commentaren op mijn werk vanuit de neurologie.

Beste Sascha, jouw enthousiasme en nuchtere, positieve instelling maakten jou een grote steun en toeverlaat. Ik heb vaak door de bomen het bos niet meer kunnen zien maar door jouw vakkundigheid op het gebied van $\mathrm{VCl}$ ben ik nooit verdwaald. Mijn dank voor de vele uren die je besteed hebt om me wegwijs te maken in de wereld van het analytisch denken, de statistiek en het 'handig opschrijven'. Onze overleguurtjes die we regelmatig in de avond of in het café hadden waren behalve 'nuttig' ook erg gezellig en ik ben blij dat we deze ook na deze dag voortzetten! 
Mijn paranimfen Ivo Aben en Mariëlle Vlooswijk. Beste Ivo, mijn dank voor het gebruik van jouw zeer zorgvuldig verzamelde gegevens, die dit boekje mogelijk gemaakt hebben. Toen ik met dit onderzoek begon gaf jij aan geen directe rol te willen vervullen, maar ik heb altijd op je terug kunnen vallen voor je hulp en advies. Ik heb veel gehad aan onze discussies. Deze dag kan ik me niet anders voorstellen dan dat jij een van mijn paranimfen bent en ik ben blij dat jij die rol op je wilt nemen.

Lieve Mariëlle, veel hebben we gedeeld in onze 'neurologie-tijd', samen met Iris en Jordie, maar vooral ook daarbuiten. Je bent dan ook een goede vriendin geworden. Ik bewonder je om je vermogen kritisch stil te staan bij de dingen en ze zo op een nuchtere en heldere manier te benaderen en een positieve zwaai te geven. In alles zie jij iets moois en dat is goed. Ik vind het erg fijn dat jij vandaag als mijn paranimf achter mij staat!

Het team heeft enige veranderingen ondergaan in de tijd. Anita Boreas, aanvankelijk ging jij dit traject in als een van mijn co-promotoren en ik wil je graag bedanken voor je betrokkenheid en al je inbreng en advies.

Samenwerken met andere disciplines en deskundigen is de sleutel tot succes. Prof. dr. H. Crijns, Jacqueline Strik, bedankt voor jullie expertise op het terrein van depressie bij patiënten die een hartinfarct doormaakten. Martin van Boxtel, Pieter Jelle Visser en Lia Baars, mijn grote dank voor jullie uitgebreide hulp met analyseren van de gegevens over APOE en ACE genotypen en jullie kritische blik en commentaar. Mijn dank ook aan Richel Lousberg, Fons Kessels en Nico Rozendaal, die mij - zeker in den beginne - erg geholpen hebben met statistische vragen en de wereld die SPSS heet. Mijn dank ook hier aan dr. M. van Vugt en de geheugenpoli voor het 'meekijken'.

Els Ketelslegers en Kitty Verwoerdt. Het was niet makkelijk iedereen op gezette tijden bij elkaar te krijgen, toch is het steeds weer gelukt dankzij jullie planning en volharding. Mijn dank voor alle ondersteuning.

ledereen bedanken, dat lukt me niet. Ik wil mijn dank uitbrengen aan alle mensen die destijds betrokken waren bij het onderzoek van Ivo Aben en Sascha Rasquin. De mensen die hun geholpen hebben bij het uitvoeren van de onderzoeken en de patiënten. Zonder deze mensen immers, stond ik hier niet. 
Beste leden van de leescommissie, bedankt voor jullie inspanningen dit proefschrift grondig te lezen en bedankt voor jullie commentaar. Het verheugt me jullie de 16 e september te mogen begroeten.

Ooit begon ik aan een loopbaan om neuroloog te worden, in het bijzonder geïnteresseerd in gedrag en cognitie, maar jouw enthousiasme voor de klinische neurofysiologie, beste Werner, deed mij zwenken naar een andere richting. Tja, ik vind dan ook altijd alles interessant. Jij, Vivianne en Danny hebben me alle ruimte gegeven voor dit onderzoek en daar ben ik jullie erg dankbaar voor. Ik ben blij dat ik deel uitmaak van een klein en hecht team dat vooruitstrevend en vernieuwend is. Een grens is een relatief en verschuifbaar begrip, en soms fictief. Daarmee wil ik ook mijn dank uitbrengen naar alle collega's van de afdeling klinische neurofysiologie die een hechte en gezellige sfeer waarborgen. Ik ben blij dat ik met jullie werk. Jongens, vanaf nu zal ik waarschijnlijk wat rustiger zijn...

Beste Paul, mijn kamergenoot en collega in hectische tijden. Je relativeringsvermogen met vrolijke noot waren niet alleen een steun voor mijn eerste wankele schreden als 'baas'. Ook wat betreft mijn onderzoek heb je me vaak een hart onder de riem gestoken. Ik blijf erbij dat je een groot gemis bent voor de neurofysiologie en de geneeskunde. Maar ik ben er ook van overtuigd dat de winst voor schrijversland minstens zo groot is.

Vrienden maken moeilijke tijden lichter en ik ben blij dat ik jullie heb. Jullie zijn met een boel, maar een aantal van jullie wil ik in het bijzonder noemen. Mijn lieve vriendinnen Nouchka, Hellen, Willemijn, Nadjah, Caroline, Kim, Ester, Linda. Goede vrienden herken je doordat ze blijven, ook als je een paar keer verhuist. Ik ken jullie al jaren en ik ben jullie dankbaar voor alle leuke tijden en luisterende oren. Lieve Harold en Heike, Rob en Margje, Jeroen en Martine, Bas en Krista, bedankt voor alle gezelligheid. Boven alles, bedankt voor jullie geduld en begrip. Zo, laten we weer eens wat leuks gaan doen!

Mijn bijzondere dank aan Camiel, Martijn, Jeroen en Anthonie. Jullie zijn als beste vrienden van Frank een soort onderdeel van ons meubilair. Ik heb veel gehad aan jullie advies en steun. Jullie staan dan ook echt altijd voor ons klaar en zijn altijd in voor een ontspannend biertje. Het wordt weer eens tijd!

Mijn schoonfamilie Ferd, Mariet, Guido, Femke en natuurlijk kleine Noortje. Bedankt voor al jullie interesse en gezelligheid. 
Mijn grote broer Giorah. Tja, het is me dan toch gelukt, maar dat had je me al eens gezegd. Als grote broer hield je van plagerij, maar altijd heb je over me gewaakt, zoals dat gaat. En je bent voor mij altijd een voorbeeld geweest. Bedankt voor alles wat je voor me bent. Binnenkort ga je trouwen en ik wens jou en Erin alle goeds van de wereld voor een mooi leven samen.

Lieve papa en mama. Het veilige en warme nest waar ik uit kom hebben me gemaakt tot wat ik ben. Van jullie heb ik geleerd dat je geen hardloper wordt als je niet hard loopt. Bedankt voor alle liefde, het onvoorwaardelijke vertrouwen in mij en dat jullie altijd achter me staan, ook als ik uitglijd.

En dan jij, mijn lieve Frank. In zoveel opzichten het tegenovergestelde van mij. Levensgenieter pur sang en broodnuchter. Precies de dingen die ik nog moest leren, al blijk ik hardleers. Mijn grote dank voor je onvoorwaardelijkheid in alles wat je voor me doet. Je hebt gelijk, het leven verdient gewoon een knipoog. Laten we dan nu het glas heffen op ons leven, samen met ons lieve knaapje Arthur.... Stimmung! 

Curriculum Vitae 


\section{Curriculum vitae}

Ariane Marie-José Johannes Bour was born on March 14th 1977 in Heerlen and grew up in the village of Limbricht. She completed her secondary education at the Gymnasium of the 'College Sittard' in 1995 and started the study Medicine at the Leiden University Medical Centre (LUMC). During this study she performed laboratory research into the immunology of dementia at the Laboratory of Gerontology and Geriatrics of the LUMC. She obtained her medical degree in 2001 and started to work as a resident in Neurology in the Leyenburg Hospital, The Hague. In 2005 she moved to Maastricht and continued her residency in Neurology at the Maastricht University Medical Centre and started the research project of this thesis in 2006. In 2008 she completed her residency neurology and now works at the Department of Clinical Neurophysiology of the Maastricht University Medical Centre. 